\title{
ELECTROMAGNETIC WAVE PROPAGATION
}

IN ALMOST PERIODIC MEDIA

Thesis by

Alan R. Mickelson

In Partial Fulfillment of the Requirements for the Degree of Doctor of Philosophy

California Institute of Technology

Pasadena, California 


\section{ACKNOWLEDGEMENTS}

For my scientific development during the early stages of my research, I owe much to my original advisor, Professor Nicholas George. While in Professor George's optics group, I often worked with Dr. David MacQuigg, a friend and colleague from whom I learned much. During this period and following this period up to the present time, I would like to acknowledge my friend and co-worker, Dr. Wàlter de Logi, for stimulating discussions and collaboration on various projects.

This present work stemmed from an idea of my present advisor, Professor C. H. Papas, whose encouragement and assistance were instrumental in all stages of this research. I also acknowledge Dr. Dwight L. Jaggard with whom I worked closely on various aspects of this work.

The style and accuracy of this work improved markedly upon careful proofreadings by my advisor, Dr. Jaggard, Dr. de Logi, and Randy Bartman. The reference lists could never have been so complete without the expert assistance of Electrical Engineering librarian Paula Samazan. Additional references were obtained from the private library of Dr. Crockett L. Grabbe.

The rough drait of this work was typed by my wife Susan, who really deserves to be acknowledged for more than typing. The expert typing of the final draft was a product of Ruth Stratton, Susan Dycus and Pat Nei11.

My financial support while at Caltech has come from Caltech, the Air Force Office of Scientific Research and the National Science Foundation. 
ABSTRACT

The problem of electromagnetic wave propagation in almost periodic media is investigated and a solution is obtained directly from Maxwell's equations. Techniques to evaluate this solution are developed. These techniques involve a generalization to almost periodic media of the Brillouin diagram of periodic media. The method of invariant imbedding is applied to the coupled mode equations which determine the Brillouin diagram for the purpose of transforming them to coupled Riccati equations. These coupled Riccati equations, when subjected to a single boundary condition, determine the solutions to both the periodic and almost periodic boundary value problems. These evaluation techniques are used to place in evidence similarities and differences of wave propagation in periodic and almost periodic media. It is shown that al though the periodic and a]most periodic theories agree in many cases of interest, there exist cases in which distinct differences appear. In cases of multi-tone perturbations, the almost periodic theory yields both simpler and more reasonable results than the periodic theory. 


\section{TABLE OF CONTENTS}

I. Introduction

A. Statement of Purpose 1

B. The Theory of Almost Periodic Functions 1

1. Historical perspective 1

2. Important concepts involved in almost periodic function theory

C. Why Bother to Study Wave Propagation in Almost Periodic Media?

D. Preview of the Following Material

II. The General Theory of Wave Propagation in Almost Periodic Structures

A. Longitudinal Media 20

$\begin{array}{ll}\text { Bibliography } & 41\end{array}$

III. Brillouin Diagrams in Almost Periodic Media 43-103

A. Basic Concepts 43

B. Brillouin Diagrams in Periodic Media - Information

1. Historical perspective 50

2. Use as a predictor 51

3. The Brillouin diagram as an ordering device 57

C. The Brillouin Diagram in Almost Periodic Media 66

D. The $\Delta k \rightarrow 0$ Limit 78 
TABLE OF CONTENTS

(continued)

Page

1. Need for a new perturbation theory 78

2. Almost periodic perturbation theory 83

3. Periodic perturbation theory 87

$\begin{array}{ll}\text { Bibliography } & 102\end{array}$

IV. The Reflection Coefficient 104-151

A. Standard Perturbation Theory for the Reflection

$\begin{array}{ll}\text { Coefficient } & 104\end{array}$

1. Setting up the exact boundary value problem 104

2. Born-Neumann series 107

3. Secularity of Born-Neumann series at resonance 108

B. The Electromagnetic Riccati Equation 109

1. Invariant imbedding - historical perspective 109

2. Stokes' recursion relation and conservation of energy

3. The electromagnetic Riccati equation 115

C. A Generalized Look at Invariant Imbedding 117

1. First order wave equations from particle counting

2. The energy conservation relations 120

3. Invariant imbedding of coupled equations 123

D. Riccati Equations, Brillouin Diagrams and Dispersion Matrices

1. Single harmonic perturbation 


\section{vi}

\section{TABLE OF CONTENTS}

(continued)

2. Almost periodic $\Delta K \rightarrow 0$ solution

3. The periodic media small $\Delta K 1$ imit

v. Conclusions

A. Relationship of the Brillouin Diagram to the to the Reflection Coefficient

B. How Periodic and Almost Periodic Media Differ 152

1. Phase relations

2. Band coalescence regime

C. The Basic Nature of Almost Periodic Structures

1. Multi-tone almost periodic perturbations

2. Almost periodic ensembles and stochastic ensembles

D. Possible Research Directions

1. Extensions to more general geometries

2. Modeling of random perturbations

3. Extensions to active media - stability criteria

4. Experimental verification of the theory

Bibliography 


\section{vij \\ TABLE OF CONTENTS \\ (continued)}

\section{Bibliography}

Page

APPENDIX D

175

APPENDIX E 


\section{1.}

Chapter I

Introduction

\section{A. Statement of Purpose}

To begin, we wish to emphasize that this report is an attempt to unify an abstract mathematical theory with wave propagation theory and not an attempt to solve a specific engineering problem. !!e feel that the results of this report could become applicable to a number of specific problems, but at the present time we are not able to predict in which areas this work will become important. The intent here is to present a theory of wave propagation in this neviy considered medium and to compare and contrast the electromagnetic properties of this medium with the properties of better understood media. In the process of the investigation, we intend to re-express certain abstract mathematical concents in engineering terminology as well as to develop new analytical techniques with which to treat wave-propagation phenomena. He hope the report achieves its purpose and, moreover, provides an interesting treatise to the reader.

We will now proceed to give the reader what we feel is the necessary mathematical history and background.

B. The Theory of Almost Periodic Functions

1. Historical Ferspective

The theory of almost periodic functions is a rather recent development. Although the theory of Fourier series was essentially 
completed by the 1820's with the work of Fourier, Poisson, Cauchy and numerous others as footnoted in Whittaker and Watson's treatise, ${ }^{1}$ it was a century later when Harald Bohr ${ }^{2-3}$ found the almost periodic generalization of Fourier series, borrowinn heavily on the quasi-periodic theory of Boh $1^{4}$ and Esclangor! ${ }^{5}$. Inspired by Bohr's set of review papers ${ }^{6-8}$, Besicovitch ${ }^{9}$, Bochner ${ }^{10}$ and Favard ${ }^{11}$ found various simplifications of the theory soon after its inception. In $1926^{12}$ and $1927^{13}$, Favard extended the theory by considering linear differential equations with almost periodic coefficients. (He later unified his research into a book ${ }^{14}$ on the topic.) other authors took a different direction from Favard, concentrating their efforts more on lifting restrictions on the class of almost periodic functions. Besicovitch ${ }^{15}$ was the first to publish a generalized theory, followed by Stepanoff ${ }^{16}$, Besicovitch and Bohr ${ }^{17}$, Linfoot ${ }^{18}$ and Wiener ${ }^{19}$. ATthough Bohr was the first to publish a book ${ }^{20}$ on the topic, he was closely followed by Besicovitch. ${ }^{21}$

Following this opening era of almost periodic theory of the 1920 's and early 1930's, there was a three-decade period of little activity in the field. However, with the dawn of the 1960's there came a deluge of papers and books on the topic. Characteristic of this period of renewed interest in the theory is Nicholas Minorsky's chapter on almost periodic solutions in his book on non-linear oscillations $^{22}$. His interest in the theory lay in the relationship between almost periodic solutions and system stability, a common 
3.

reason for renewed interest in the topic. To completely review the developments in this field in the last decade and a half is beyond the scope of this report as is evidenced by the length of the extensive, but not exhaustive, bibiography in A. M. Fink's set of lecture notes on almost periodic differential equations ${ }^{23}$. One author's work is of importance in relation to our study. That would be the two papers ${ }^{24-25}$ of Jacob Abel who first considered the almost periodic Mathieu equation, the same equation to be considered in this work. Abel ran across the equation while engaging in a study of stability of elastic systems.

2. Important Concepts Involved in A.1most Periodic Function Theory Ve begin this section with a short qualitative discussion of almost periodic functions before jumping to the more abstract elements of the theory. Perhaps the most direct way of obtaining a feeling for almost periodic functions is to compare them with their close relatives (actually a direct subclass), the periodic functions. Consider the periodic function

$$
f_{p}(z)=\cos \pi z+\cos 3 \pi z+\cos 5 \pi z
$$

and an almost periodic counterpart

$$
f_{A p}(z)=\cos \pi z+\cos 3 \frac{1.7}{\sqrt{3}} \pi z+\cos \frac{5 \sqrt{3}}{1.7} \pi z
$$

These functions are plotted in figure (1).

For the first few periods of (1), the functions don't seem to 

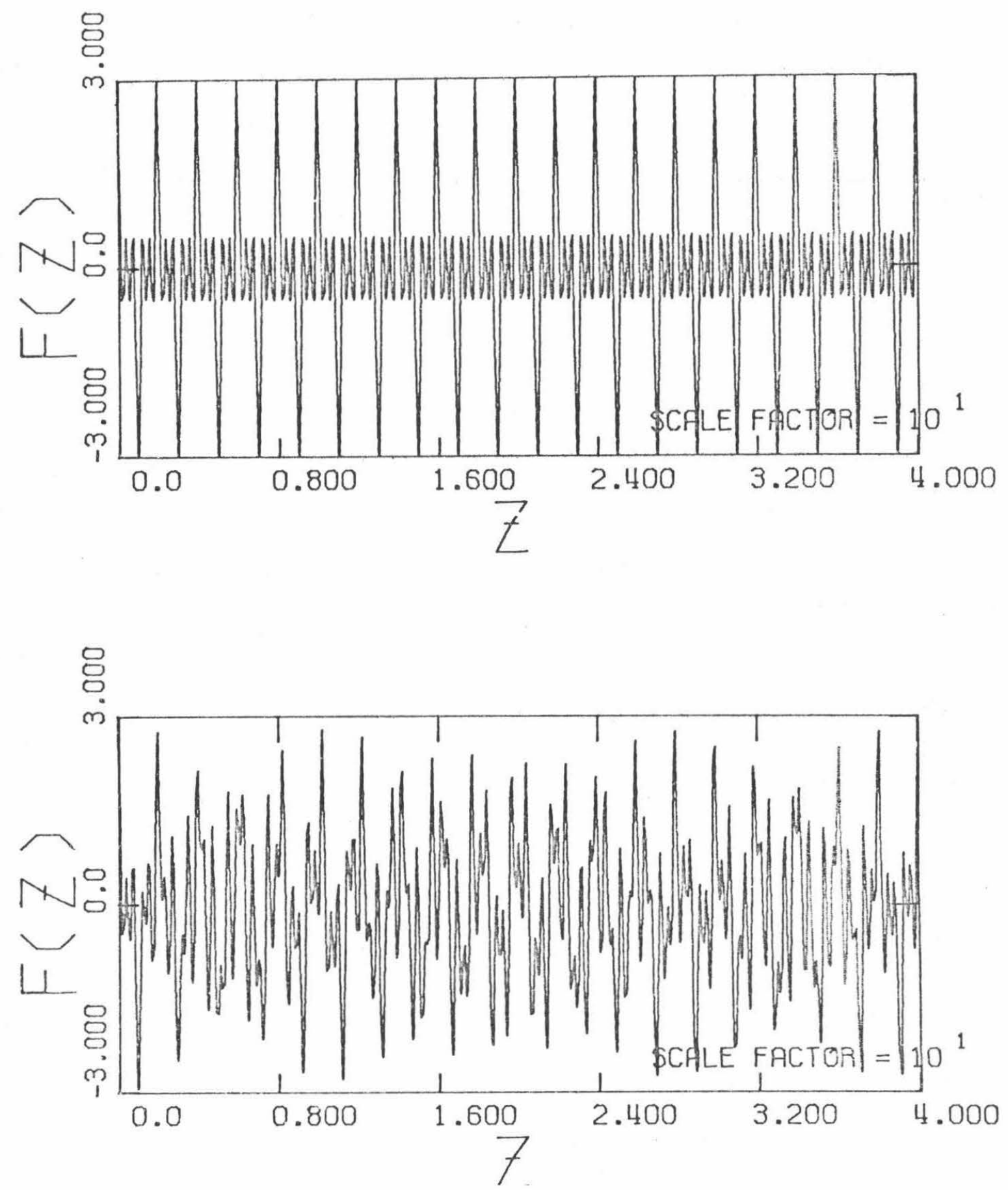

Figure 1.1. a) A plot of the periodic function $f_{p}(z)=\cos \pi z+\cos 3 \pi z$ $+\cos 5 \pi z$. b) A plot of the almost periodic function $f_{A P}=\cos \pi z+$ $\cos 3 \frac{1.7}{\sqrt{3}} \pi z+\cos \frac{5 \sqrt{3}}{1.7} \pi z$. 
differ too much. Farther on they certainly do, in spite of the fact that their arguments differ by only a small amount. The almost periodic function appears to have o. wandering phase with respect to the periodic one. This is indeed the case as we notice that

$$
\frac{3 \sqrt{3}}{1.7}=3+I R
$$

where IR is an irrational number. He further note that we could write (2) in the form

$$
\begin{aligned}
& f_{A P}(z)=\cos \pi z+\cos \left(3 \pi z+\phi_{2}\right)+\cos \left(5 \pi z+\phi_{3}\right) \\
& \phi_{2}=I R_{2} \pi z \quad \phi_{3}=I R_{3} \pi z
\end{aligned}
$$

That IR is irrational is a fundamental point. If, in a sum of trigonometric functions, the ratios of their frequencies can be written as rational fractions, e.g. in the two-tone case

$$
\begin{aligned}
& f_{p}(z)=\cos k_{1} z+\cos k_{2} z \\
& \frac{k_{1}}{k_{2}}=\frac{p}{q} \\
& p, q \text { relatively prime integers }
\end{aligned}
$$

a period for the system can be found, and in fact the period is just $2 \pi$ times $p$ divided by $\kappa_{7}$, which is equal to $2 \pi$ times $q$ divided by $\kappa_{2}$. If the ratio is irrational, however, it is easily shown ${ }^{26}$ that no period can exist. The function can come close to repeating at 
values of $z$ there rational approximations to the irrational ratio of $k_{1}$ to $k_{2}$ satisfy the above stated periodic condition, but the function can only come close, as $2 \pi$ times $p$ divided by $k_{7}$ is no longer equal to $2 \pi$ times $q$ divided by $\kappa_{2}$. The small difference in these two almost periods will cause one or the other of the cosines to not truly repeat.

We now feel we are ready to approach the topic in a slightly more rigorous language for purposes of later exposition. We will use Bohr's ${ }^{27}$ definition of almost periodicity.

Definition 1.

Let $S(\tau)=\{\tau|| f(t+\tau)-f(t) \mid<\varepsilon \forall t\}$.

Then $f(t)$ is an almost periodic function iff $S(\tau)$ for every $\varepsilon>0$, is relatively dense.

Definition 2.

A set of $S$ on $R$ is called relatively dense if there exists a positive $\mathrm{L}$ such that

$$
[a, a+L] \Omega S \neq \phi \quad \forall a \varepsilon R
$$

A table of symbols for the definition is included in table (1).

It is shov $n^{27}$ from Definition 1 that any almost periodic function is uniformly continuous. It is also apparent from the definition that an almost periodic function must have continually vacillating values $^{28}$ lest there be no almost periods in some regime. But a function possessing these two attributes must in some sense be stable,as 
TABLE I

\begin{tabular}{|c|c|}
\hline Symbol & Meaning \\
\hline\{\} & the set of \\
\hline | & such that \\
\hline$|f(x)|$ & the absolute value of $f(x)$ \\
\hline$\forall$ & for all \\
\hline ( ) & the open interval \\
\hline[] & the closed interval \\
\hline$u$ & the union of \\
\hline$\Omega$ & the intersection of \\
\hline$\phi$ & the null (empty)set \\
\hline $\mathrm{R}$ & the set of real numbers \\
\hline$A \supset B$ & A contains B \\
\hline$A \subset B$ & $A$ is contained by $B$ \\
\hline $\begin{array}{l}\text { TABLE I. } \\
\text { IN THIS } \\
\text { SYMBOLS. }\end{array}$ & $\begin{array}{l}\text { HEMATICAL SYMBOLS EMPLOYED } \\
\text { WITH THE MEANING OF THE }\end{array}$ \\
\hline
\end{tabular}


its values over some finite interval of time is "about the same" as its values at any other time. The definition therefore makes clear that the existence of almost periodic solutions to differential equations governing the output of machines or orbits of celestial bodies somehow implies the stability of the machine or the stability of the orbit. He can now understand one reason for the resurgence of interest in almost periodic functions.

For further discussion we find it useful to use the results of the basic theorem of almost periodic functions, that is to say:

\section{Theorem 1.}

Any almost periodic function $f(t)$ is expressible as a generalized Fourier series

$$
f(t)=\sum_{N} a_{N} e^{i \lambda N}
$$

or more precisely, if for any given $\varepsilon, a_{M}$ and $\lambda_{N}$ can be found such that

$$
\left|f(t)-\sum_{N} a_{N} e^{i \lambda_{N} t}\right|<\varepsilon
$$

then $f(t)$ is an almost periodic function. This theorem has immediate consequences concerning composites of almost periodic functions. Consider the addition of two almost periodic functions. If we find 


$$
\begin{aligned}
& f(t)=\sum_{N} a_{N} e^{i \alpha_{N} t} \\
& g(t)=\sum_{N} b_{N} e^{i \beta_{N} t}
\end{aligned}
$$

then we can write

$$
\begin{aligned}
f(t)+g(t) & =\sum_{N_{g} N^{\prime}}\left(a_{N^{\prime}} e^{i \alpha_{N} t}+b_{N^{\prime}} e^{i \beta_{N} t^{t}}\right) \\
& =\sum_{M} c_{11^{1}} e^{i \gamma_{M 1} t}=h(t)
\end{aligned}
$$

and therefore we find that the sum of two almost periodic functions is also an almost periodic function. similarly, we could show that differences, products, quotients, integrals, and derivatives of almost periodic functions are also almost periodic (with suitable restrictions). It is then true that almost periodic functions are a general class of functions which is closed under certain operations. Implicit also in Theorem 1 is the inclusion of the periodic functions as a subclass of the almost periodic functions as can be seen by making the replacement

$$
\lambda_{N} \rightarrow N \lambda
$$

in equation (5).

One more set of defintions will be included here for the usefulness of the terminology in discussing solutions of differential equations with almost periodic coefficients. 


\section{Definition 3}

The exponent exp of an almost periodic function $f(t)$ is defined as the set of $\lambda$ 's contained in its generalized Fourier series, i.e., if

$$
f(t)=\sum_{N=0}^{\infty} a_{N !} e^{i \lambda_{N} t}
$$

then

$$
\exp [f(t)]=\left\{\lambda_{0}, \lambda_{1}, \lambda_{2}, \ldots\right\}
$$

Definition 4

The module $M$ of the $\exp [f(t)]$ is the closure of the set under addition, i.e., if

$$
f(t)=a_{1} e^{i \lambda_{1} t}+a_{2} e^{i \lambda_{2} t}
$$

then

$$
M[\exp [f(t)]]=\left\{b_{M ! !} \mid b_{M M !}=M \lambda_{1}+M ! \lambda_{2}, \forall I 1, M\right\},
$$

\section{Defintion 5}

An almost periodic function $g(t)$ is modularly contained by $M[\exp [f(t)]]$ iff

$$
M[\exp [g(t)]] \quad C \quad M[\exp [f(t)]]
$$

The usefuiness of these defintions may at first seem obscure. However, a simple example should prove their usefulness. He consider the case of a differential equation with periodic coefficients which can be written in the form 


$$
D f+A f=0
$$

where $D$ is a differential operator and $A$ a periodic function of basic period $k$. Say that we know, from some very general techniques, that a solution exists with the same period as A. He can then always write the solution in the form

$$
f(t)=\sum a_{N} e^{i N k t}
$$

where the $a_{N}$ 's can be determined from a consistency requirement. We would like to be able to generalize this argument to enable us to solve systems of the form of equation (10) where $A$ is an almost periodic function, say for simplicity a two-tone almost periodic function expressible in the form

$$
A(t)=b_{1} e^{i \kappa_{1} t}+b_{2} e^{i \kappa_{2} t}
$$

We wouldn't know how to look for a solution of the same almost period as the term "almost period" is stil1 i11-defined. However, we could look for a "modular containment" solution, i.e. a solution of the form

$$
f(t)=\sum_{N, M} a_{N M} e^{i N K_{1} t+M K_{2} t}
$$

where the a's, as in the argument above, are to be determined from a consistency argument. The above argument is a simplification of the argument that will appear in chapter two. 
C. Why Bother to Study llave Propagation in Almost Periodic Media?

To this point the development has seemed mathematical beyond the scope normally required for engineering application. The natural question in the reader's mind is why bother with all this mathematics if no application is imminent? We wish to allay the reader's fears by discussing briefly some promising areas of application.

Before listing these areas, though, we wish to present a brief discussion to motivate our reasoning. We predicate this discussion on the fact that "real" electromagnetic (or optical) devices are finite in length although periodic devices tend to contain many periods within this length. Further, these "periods" are never perfect in practice. Whether they be due to amplitude wobble, phase wobble, cracks in the structure or various other causes, there are always small perturbations to destroy the "perfection" of the cellular structure of the device. A wave in traversing such a media, therefore, sees many almost identical cells. But an almost periodic structure would look, at least qualitatively, much like this to the wave, as the almost periodic structure comes close to, but does not quite achieve, a "perfect" cellular structure. The almost periodic structure seems to take imperfections into account and in this sense is actually a more realistic model than a perfectly periodic structure.

But is the increased effort required to evaluate an almost periodic solution worth the effort? Although we don't know the 
answer to that question in general, we do know of cases in which the almost periodic solution is simpler than the periodic solution. (Such cases will be considered in chapters three and four.) !le conclude that if one believes that almost periodic modeling is in some sense more realistic than periodic modeling, then there are certain cases in which the almost periodic modeling is worth the effort.

le will now list and briefly discuss some possible applications.

\section{Stochastic Perturbations}

Most random media propagation theory requires some type of ensemble averaging which may not always be desirable. For example, consider a fiber optic link in which we wish to compute some quantity, such as the amount of mode-coupling due to stochastic perturbations.

Ensemble averaging would correspond to averaging over many similarly degraded links, whereas in practice we will use but one link. In general, the stochastic solution would correspond to finding the first and second moments of a distribution function which is too complex to obtain. But in certain cases (i.e., broad distributions) the averaged result does not necessarily correspond very closely to the situation in any one given link. In these cases, to obtain more reasonable bounds on operational characteristics, it would be desirable to solve the problem for a single member of the ensemble. As will be discussed in more detail in chapter five of this work, a natural choice for an ensemble member would be a 
specially constructed almost periodically perturbed structure. By solving this case deterministically, with free parameters, good bounds could be obtained for operational characteristics.

\section{Integrated Optics}

A1though to this point in the technological development of integrated optics most of the periodic structure devices have operated very much as the periodic theory predicts they should, the possibility remains that at some future date manufacturing limitations may dictate that the theory be modified to agree with the experiment. Under the assumption that almost periodic analysis, in some sense, requires less restrictive assumptions than periodic analysis, the almost periodic theory could provide a useful analytic technique for predicting the limiting precisions with which devices must be fabricated, or even the theory could suggest ways to avoid technological 1 imitations. Perhaps, in certain cases, almost periodic devices will be preferable to their periodic counterparts.

\section{Crystal Lattice Disorder Theory}

Work on the application of almost periodic function theory to the theory of disordered crystal lattices has already been initiated in the work of Romerio ${ }^{29}$ and Balanis. ${ }^{30}$ We feel a good direction in which to continue would be to apply almost periodic Brillouin diagram analysis (developed in chapter three of this work for the one dimensional case) to some simple cases, following the lead of slater ${ }^{31}$ who 
15.

so carefully and clearly treated the periodic lattice. We feel that it is possible by gaining better insight into almost periodic phonon modes to better understand the collective quantum processes which cause solid state phenomena.

We believe the above listing represents only a sample of the areas of possible utility of almost periodic wave propagation but we leave our discussion at this point so as not to unduly lengthen this report.

\section{Preview of the Following Material}

In chapter two we solve the problem of wave propagation in longitudinally varying almost periodic media. The solution appears as a generalization of Floquet's theorem for periodic media and is therefore compared in structure to this familiar theory.

In chapter three the Brillouin diagram of periodic media is generalized to the almost periodic case. The chapter begins with a brief discussion of dispersion relations and diagrams, and an historical perspective on the development of the Brillouin diagram in periodic wave propagation. The inclusion of this non-original material is justified on the basis that it expedites the discussion of almost periodic wave propagation by highlighting the underlying concepts and techniques. The main developments of the chapter 7 ie in the generalization of the Brillouin diagram technique to almost periodic media, and the detailed comparison of almost periodic wave 
propagation with periodic wave propagation employing this newly developed method.

In chapter four a technique is developed to calculate reflection coefficients of finite logitudinally varying almost periodic media. As in chapter three, this chapter also begins by discussing a certain amount of non-original, yet basic, material. The secularity of standard perturbation expansions is illustrated and the method of invariant imbedding, a powerful technique of avoiding secularity, is developed in a more general way than has previously been accomplished. The accomplishments of the chapter include numerical comparisons of solutions of the exact Riccati equation for periodic and almost periodic media and the development of a technique for generating reflection coefficients for "practical" media.

Chapter five is devoted to discussion and conclusions. Possible research problems designed to extend and verify the theory are suggested.

We are presently ready to embark on our exposition of the theory of wave propagation in almost periodic structures. 
Bibliography for Chapter One

1. Ihittaker, E. T. and G. N. Watson, A Course of Modern Analys is (London: Cambridge Univ. Press, Fourth Edition - 1927, reprinted - 1973). The references alluded to appear on page 160.

2. Bohr, H., Comptes Rendus 177, 737-739 (1923).

3. Bohr, H., Comptes Rendus 177, 1090-1092 (1923).

4. Boh1, Bu11. Soc. Math. de France 38, 5-144 (1910).

5. Although Bohr acknowledges Esclangon as being in part responsible for the quasi-periodic theory, there seems to be no readily accessible work by him. References we have seen given are:

Esclangon, Annales de 1'Observ. de Bordeaux (1919) and Esclangon, 11. PhD. Thesis, France (1904). It seems that a portion of Esclangon's contribution 1 ies in his coinage of the term quasiperiodic as Bohl uses no such classification.

6. Bohr, H., Acta Math. 45, 29-127 (1924).

7. Bohr, H., Acta Math. 46. 101-214(1925).

8. Bohr, H., Acta Math. 47, 239-281 (1926).

9. Besicovitch, A. S., Acta Math. 47, 283-295 (1926).

10. Bochner, S., Proc. London Math. Soc. 26, 433-452 (1926-1927).

11. Favard, J., Comptes Rendus 182, 757-759 (1926).

12. Favard, J., Comptes Rendus 182, 1122-1124 (1926).

13. Favard, J. Acta Math. 51, 31-81 (1927). 
18.

14. Favard, J., Fonctions Presque-Periodiques (Paris: Cahiers Scientifiques, 1933).

15. Besicovitch, A. S., Proc. London Math. Soc. 25, 495-562 (1926).

16. Steppanoff, H., Math. Ann. 95, 473-498 (1928).

17. Besicovitch, A. S. and H. Bohr, J. London Math. Soc. $\underline{3}, 172-176$ (1928).

18. Linfoot, E. H., J. London Soc. 3, 177-182 (1928).

19. Uiener, N.,Acta Math. 55, 117-258 (1930). This work is reprinted in: Wiener, N.,Generalized Harmonic Analysis-Tauberian, Theorems (Cambridge, M.I.T. Press, 1951).

20. Bohr, H., Almost Periodic Functions (New York: Chelsea, 1951).

21. Besicovitch, A. S.,Almost Periodic Functions (London: Cambridge University Press, 1932).

22. Minorsky, N., Nonlinear Oscillations (Muntington, N.Y.: R. E. Kreiger Publishing Co., original ed. - 1962, reprinted - 1974).

23. Fink, A. M., Almost Periodic Differential Equations (Berlin: Springer-Verlag), 1974).

24. Abe1, J., Q.J. of App. Math. 28, no. 2, 205-217 (1970).

25. Abe1, J., J. Math. Anal. and App1. 36, 110-122 (1971).

26. Bedrosian, G., PhD Thesis, C.I.T. (1976). See pp. 106-107.

27. Definitions and proof of uniform continuity are presented in Fink's book (23) on pages 7-9.

28. We have excluded the trivial case of a constant function. 29. Romerio, M. V., J. Math Phys. 12, no. 3, 807-835 (1971). 
30. Balanis, G., unpublished paper (1977).

31. Slater, 1. C., Phys. Rev. 87, no. 5, 807-835 (1952). 
20.

\section{Chapter II}

The General Theory of Wave Propagation in Almost Periodic Media

\section{A. Longitudinal Media}

The motivation for the derivation contained in this chapter comes in large part from the well known Floquet theory ${ }^{1}$, so thoroughly discussed in Whittaker and Watson's treatise ${ }^{2}$. However, the almost periodic generalization of Floquet's theory can neither be proved or evaluated by techniques analogous to those applied to the periodic case. For these reasons, the development herein contained will investigate the two theories simultaneously from a new vantage point which naturally allows for illumination of the nature of the generalization.

To begin, let us remind ourselves of Floquet's result. Given the differential equation with periodic coefficients, e.g., the second order equation

$$
\frac{d^{2} \psi}{d z^{2}}+k^{2}(z) \psi=0
$$

where $k$ is a periodic function of the variable $z$ with period $k$, Floquet shows that $\psi$ can be written in the form

$$
\psi=f(z) P(z)
$$

where $P$ is a periodic function of $z$ with period $k$ and $f$ is a propagation factor which is to be determined. This result could be stated 
for the case of electromagnetic wave propagation as follows: the wave solution to Maxwell's equations in a periodic medium can always be written as a product of a propagation factor and a term involving the periodicity of the medium. We wish to find an almost periodic generalization of this theory.

We now consider the problem of wave propagation inside a doubly infinite slab of a medium whose index of refraction varies only with the coordinate $z$ (refer to figure 1). We will consider only plane wave solutions with propagation vector in the z-direction and transverse polarization. For such a case, the wave equation is easily derived from Maxwel1's equations ${ }^{3}$, and found to be

$$
\frac{d^{2} \psi}{d z^{2}}+k^{2}(z) \psi=0
$$

where $\psi$ denotes either the $\mathrm{x}$ or $\mathrm{y}$ polarized component of the $\mathrm{E}$ vector and $k^{2}(z)$ is defined by

$$
k^{2}(z)=\frac{\omega^{2}}{c^{2}} \varepsilon(z)
$$

where $\omega$ is the angular frequency of the wave and $c$ the speed of light in vacuum. We choose the medium's dielectric constant $\varepsilon$ to be a real (10ssless) function of $z$, as the generalization to complex refractive index should be straightforward, as it is in the periodic case $^{4-5}$. We further wish to consider cases in which $\varepsilon$ is either a periodic or almost periodic function of $z$ and therefore expressible 
22.

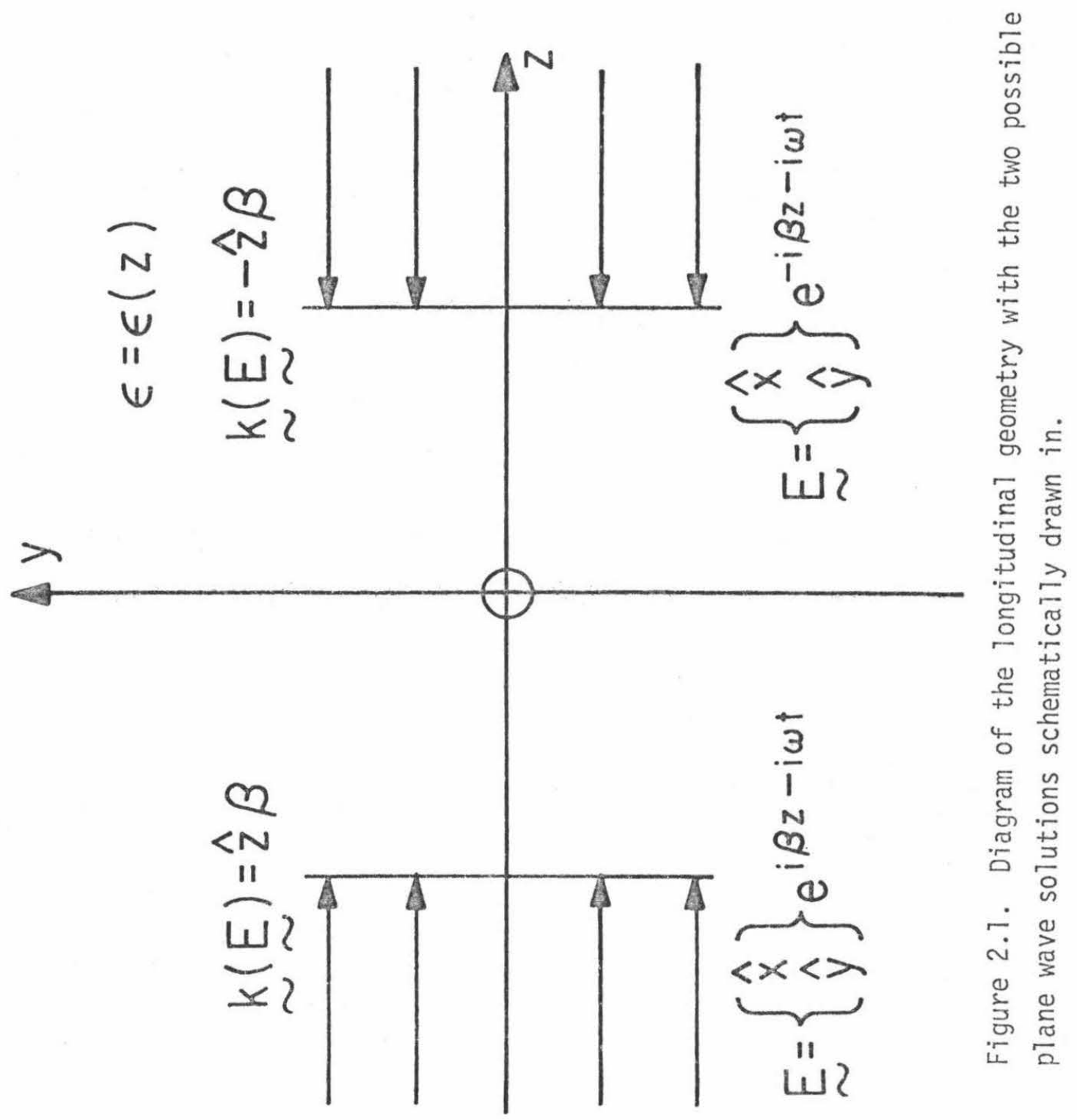


as

$$
\varepsilon(z)=\varepsilon_{r}\left\{\sum_{N=1}^{+N_{T}} \frac{1}{2} n_{N} e^{i \kappa_{N} z}+\sum_{N=-N_{T} \frac{1}{2} n_{N}}^{-1} e^{-i \kappa}-N^{z}+1\right\}
$$

where reality of $\varepsilon$ requires

$$
n_{i}=n_{-i}^{*}
$$

where the asterisk denotes complex conjugation. In the periodic case, the $K^{\prime} s$ must satisfy the relation

$$
K_{N}=N_{K}
$$

whereas in the almost periodic case

$$
\frac{k_{i}}{k_{j}}=\text { IR for at least one pair of } i, j(i \neq j)
$$

where IR represents an irrational number. To simplify the present discussion we will assume all n's to be purely real, although this restriction will be relaxed in the discussion of chapter III.

For present and future use we wish to define the Fourier transform pair

$$
\begin{aligned}
& f(z)=\frac{1}{2 \pi} \int_{-\infty}^{\infty} \hat{f}(\gamma) e^{i \gamma z} d \gamma \\
& \hat{f}(\gamma)=\int_{-\infty}^{\infty} f(z) e^{-i \gamma z} d z
\end{aligned}
$$


where theorems concerning transform relationships to be employed in present and future arguments can be found in any of numerous references $^{6-8}$.

We now apply the Fourier transform (9b) to equation (3) where the $\varepsilon$ is expressible as in (5) to obtain

$$
-\gamma^{2} \hat{\psi}(\gamma)+\frac{k^{2} \varepsilon_{r}}{2} \sum_{N=-N_{T}}^{+N} n_{N} \hat{\psi}\left(\gamma-\kappa_{N}\right)=0
$$

where we have reduced the separate summations of (5) to a single summation by defining $\kappa_{-N}=\kappa_{N}, n_{0}=2, k_{0}=0$. We have now reduced $a$ second-order differential equation to a non-local algebraic equation, a type of equation whose solution we do not know how to find in general. However, we can reduce the non-local equation to an infinite set of local equations by assuming a solution of the form

$\hat{\psi}(\gamma)=\sum_{M_{1}}^{+\infty} \sum_{-\infty}^{+\infty} \ldots M_{2}=-\infty \dot{M}_{N_{T}}^{+\infty}{ }_{=-\infty}^{+\infty} a_{1}, M_{2} \ldots, M_{N_{T}} \hat{f}\left(\gamma+M_{1} K_{1}+M_{2} K_{2}+\ldots+M_{N_{T}} K_{N_{T}}\right)$

and equating the coefficients of $\hat{f}$ at each given argument to zero. It is not necessarily true that this procedure will work, but we will assume it does and later show it yields a reasonable result. For concreteness we assume that the dielectric constant has but two "tones" in its spectrum in the almost periodic case, i.e., 


$$
\begin{gathered}
N_{T}=2 \\
n_{1}=n_{-1} \\
n_{2}=n_{-2} \\
n_{0}=2 \\
\varepsilon(z)=\sum_{r=N_{T} \sum_{i=-N_{T}}} e^{i \kappa_{i} z}=\varepsilon_{r}\left[1+n_{1} \cos \kappa_{1} z+n_{2} \cos _{2} z\right]
\end{gathered}
$$

but any number of tones in the periodic case. The tones in the periodic case, however, must satisfy equation (7). Using our above ansatz we see the solution should be written

$$
\begin{aligned}
& \hat{\psi}(\gamma)=\sum a_{N} \hat{f}\left(\gamma+N_{K}\right) \quad \text { periodic } \\
& \hat{\psi}(\gamma)=\sum a_{N M} \hat{f}\left(\gamma+M_{K_{1}}+N \kappa_{2}\right) \quad \text { almost periodic }
\end{aligned}
$$

To solve the resulting equation we could simply equate the coefficients of $f\left(\gamma+N_{K}\right)$ for each $N$ and solve the resulting infinite set of coupled algebraic equations. We elect not to do this in transform space but to inverse transform and consider our result in configuration space. The resulting forms of solution are

$$
\begin{aligned}
& \psi(z)=\sum_{N} a_{N} e^{i N k z_{f}(z)} \quad \text { periodic } \\
& \psi(z)=\sum_{N, M} a_{N M} e^{i\left(M_{K} z^{z+N K} 2^{z)}\right.} f(z) \text { almost periodic }
\end{aligned}
$$

Not too surprisingly, equation (14a) is exactly a statement of 
Floquet's theorem and perhaps in (14b) we have found its almost periodic generalization. We feel the result is at least plausible in the following sense. In Floquet's theory we find the form of solution to be the product of a propagation factor with a factor involving the periodicity of the medium, in fact, the factor being just the "modular containment" of the basic period. But in these terms this is exactly what the almost periodic solution contains, the product of a propagation factor and the modular containment of the almost periods. The result agrees in form with that found by Abe1, 9-10 who employed a different method in his derivation. However, we have yet to show either existence or convergence of our scheme. We wish to show that the $\mathrm{a}_{\mathrm{NM}}$ 's die out for sufficiently large $\mathrm{N}$ and $\mathrm{M}$. We assume that the propagation constant does not go to zero (or at least we are considering a frequency range in which it does not go to zero) allowing us to divide both sides of (14b) by $f$, resulting in

$$
g(z)=\frac{\psi(z)}{f(z)}=\sum_{N, M} e^{i\left(M \kappa_{1}+N \kappa_{2}\right) z}
$$

We now define the mean value operator by

$$
M\{Q(z)\}=\lim _{T \rightarrow \infty} \frac{1}{2 T} \int_{-T}^{T} Q(t) d t
$$

Taking the square modulus of both sides of (15) and applying the mean value operator of (16), we find the Parseval type relation 


$$
M\left\{|g(z)|^{2}\right\}=\underset{N, M}{\sum_{N}}\left|a_{N M}\right|^{2}
$$

Let us consider this result. From energy considerations we would expect the mean value of the squared modulus of $\psi$ (the energy density of the phase front) to be a bounded quantity and we have already restricted ourselves to cases in which the propagation factor is bounded away from zero. Accordingly, the lefthand sịde of (17) is taken to be a bounded quantity. The conclusion is that only a finite number of the $a_{N M}$ 's can be "appreciable" in order to satisfy (17). Therefore, we can safely assume that the $a_{N M}$ 's die out for sufficiently large $N$ and $M$ and the system of (15) can be effectively truncated. Having convinced ourselves of the plausibility of the ansatz contained in (14b) we proceed to investigate the structure of the resulting theory. The problem we have to solve to obtain a general solution to (3) is to find the function $f(z)$. We redefine our unknown by setting

$$
f(z)=e^{i \beta z}
$$

where $\beta$ is now the variable. Using (18) in (14b) and substituting in (3) using (12) we find 


$$
\begin{aligned}
& \sum_{M, N}\left[k^{2} \varepsilon_{r}-\left(\beta+M k_{1}+N \kappa_{2}\right)^{2}\right] a_{M N} e^{i\left(\beta+M k_{1}+N k_{2}\right) z} \\
& +\frac{k^{2} \varepsilon_{r}}{2} \sum_{j, l}\left[n_{1} e^{i\left(\beta+(j+1) k_{1}+\ell \kappa_{2}\right) z}+n_{-1} e^{i\left(\beta=(j-1) k_{1}+\ell k_{2}\right) z}\right. \\
& \left.+n_{2} e^{i\left(\beta+j \kappa_{1}+(\ell+1) \kappa_{2}\right) z}+n_{-2} e^{i\left(\beta+j \kappa_{1}+(\ell-1) \kappa_{2}\right) z}\right]=0
\end{aligned}
$$

As we have $\kappa_{1}$ and $\kappa_{2}$ non-commensurable, we can adopt a technique used in generalized harmonic analysis ${ }^{11}$ to simplify the result. We simply multiply by the kernel

$$
A=e^{i K z} \frac{1}{2 T}
$$

and integrate from $-T$ to $T$ taking the 1 imit for $T \rightarrow \infty$. This procedure removes both the complex exponentials and the summation signs and leaves us with the doubly infinite set of algebraic equations

$$
\begin{aligned}
& {\left[k^{2} \varepsilon_{r}-\left(\beta+M k_{1}+N \kappa_{2}\right)^{2}\right] a_{M N}+\frac{n k^{2} \varepsilon_{r}}{2}\left[n_{1}\left(a_{M-1, N}+a_{M+1, N}\right)\right.} \\
& \left.+n_{2}\left(a_{M, N-1}+a_{M, N+1}\right)\right]=0
\end{aligned}
$$

where we have chosen $n_{-1}$ to be equal to $n_{1}$ and $n_{-2}$ to be equal to $n_{2}$. This is the set of equations of which we spoke earlier when perusing the non-1ocal equation (10) in transform space. The set is homogeneous and it is useful to recast it in a matrix form. As the $a_{M N}$ 's are a countable set, we know that we can always map them into a one-dimensional vector and thus write (21) in a familiar form 


$$
\underset{\sim}{\sim} \underset{\sim}{\mathrm{a}}=0
$$

where $\underset{\sim}{a}$ is the vector obtained by mapping the $a_{M N}$ 's and $\underset{\sim}{\sim}$ is the corresponding matrix of coefficients. Clearly then the condition $\beta$ must satisfy is

$$
\operatorname{det} \underset{\sim}{\mathrm{D}}=0
$$

and by backsubstituting in (22) these values of $\beta$, we can obtain all the a's as a one-parameter family $y^{12}$, Before discussing how to perform this feat, however, it is instructive to examine the analogous situation in the periodic case.

In the periodic case the wave equation is found to take the form of (22) with $\underset{\sim}{\mathrm{D}}$ defined as ${ }^{13}$

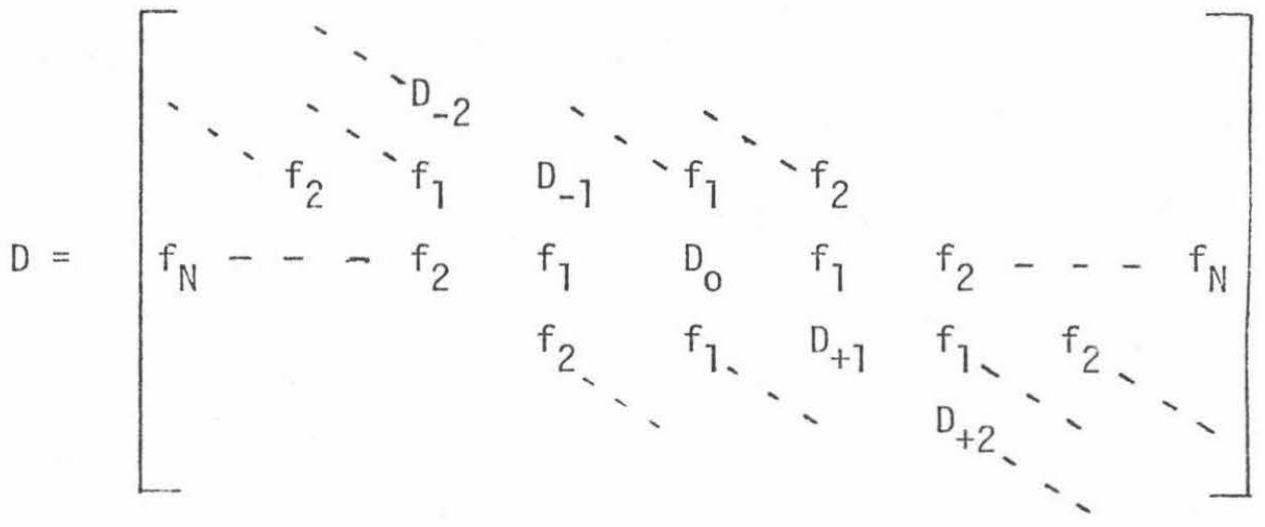

$$
\begin{aligned}
& D_{N}=1-\frac{(\beta+N K)^{2}}{k^{2} \varepsilon_{r}} \\
& f_{N}=n_{N} / 2
\end{aligned}
$$


30.

where all the elements of the matrix have been normalized by $k^{2} \varepsilon_{r}$. Evidently, we are faced with the impossible task of evaluating an infinite determinant. We see that the matrix must somehow be truncated if we are to obtain a useful approximation. But it is equally clear that the infinite determinant cannot be uniformly convergent as its diagonal grows with its dimension $N$, casting uncertainty on any truncation scheme. $\mathrm{Hill}^{14}$ circumvented this problem, however, by a clever argument so aptly treated in Whittaker and Watson ${ }^{2}$ and obtained an alternative dispersion relation,i.e, a relation connecting $\beta$ with $k$, of the form

$$
\begin{aligned}
& \sin ^{2}\left(\frac{\pi \beta}{k}\right)=\Delta(0) \sin ^{2}\left(\frac{\pi k \sqrt{\varepsilon_{r}}}{\kappa}\right)
\end{aligned}
$$

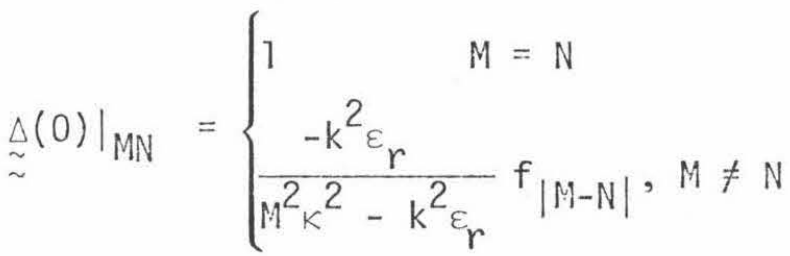

$$
\begin{aligned}
& \Delta(0)=\operatorname{det} \underset{\sim}{\Delta} \underset{\sim}{\Delta}(0)
\end{aligned}
$$

Perhaps the major achievement of this approach is to explicitly illustrate the "we11-behavedness" of the theory. Clearly, for each k there exist an infinite number of $\beta$ 's telling us that no pathology can render this dispersion relation unsolvable, and hence (25) acts as an existence thcorem. Further, $\Delta(0)$ is now uniformly convergent for practically all values of $k^{2} \varepsilon_{r}$ (Ref.15) and therefore can be truncated with gay abandon.

Looking back at the almost periodic case, we see a bleak picture. As the mapping of a plane to a line is non-unique, we must use 
care in implementing our approach so as not to lose important terms. Until we have defined this mapping from a plane to a line, we don't even know the form of $\underset{\sim}{\mathrm{D}}$, much less have a Hill's determinant-type theory, Further, we can already see we are plagued with the same lack of uniform convergence so evident in (24), as our almost periodic diagonal elements are of the form manifested in (24). However, having come this far already, we push forward unrelentingly.

We now wish to define our plane-to-7ine mapping, with the motivation for our technique to come later. The basic scheme is illustrated in figure 2, A grid is drawn with the points labeled by their integer coordinates $(i, j)$. The boxes are formed by 1 ines passing through points equidistant from the origin $(0,0)$ in the sense that the distance from point $1\left(i_{1}, j_{1}\right)$ to point $2\left(i_{2}, j_{2}\right)$ is defined by

$$
d_{12}=\left|i_{1}-i_{2}\right|+\left|j_{1}-j_{2}\right|
$$

We label the boxes 1,2,3, etc., according to the order in which they would be traversed in traveling outward from the origin, analogously to the labeling of Brillouin zones in a plane ${ }^{16}$. We say the points on and within the $i^{\text {th }}$ box are the $i^{\text {th }}$ order points. Further, we define the points to the right of the origin to include those points directly below the origin and the points to the left of the origin to include those points that lie directly above the origin. To specify a given order mapping, it remains only to specify the order in which the points to the right and the points to the left must be 


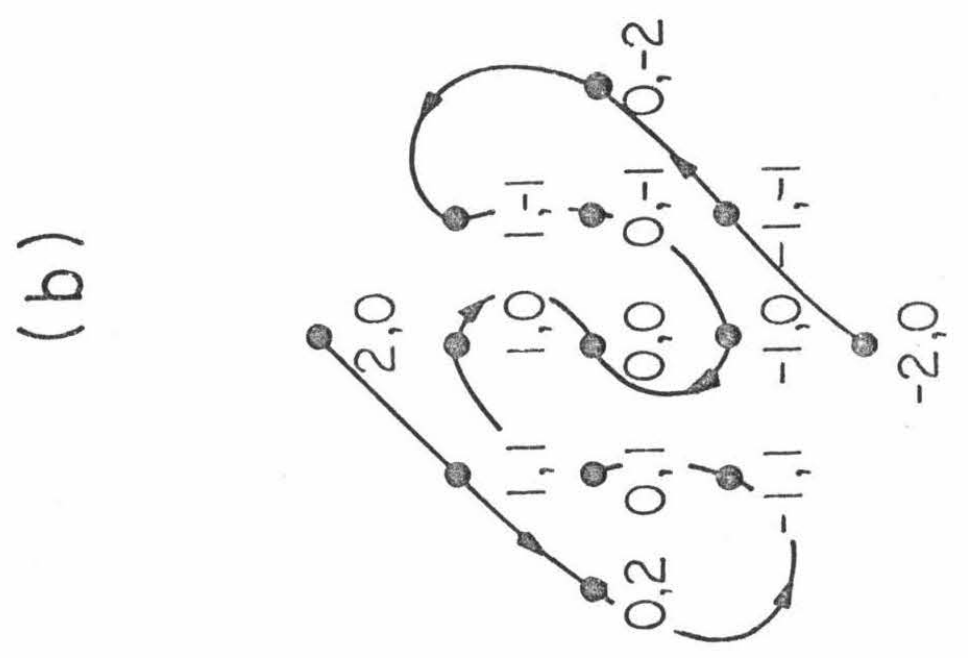

$+$

$=\frac{\square}{4}$

数 4

ป

"ू के

产

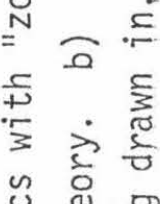

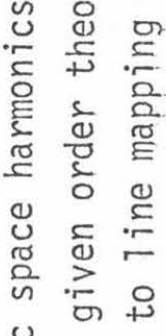

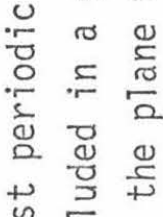

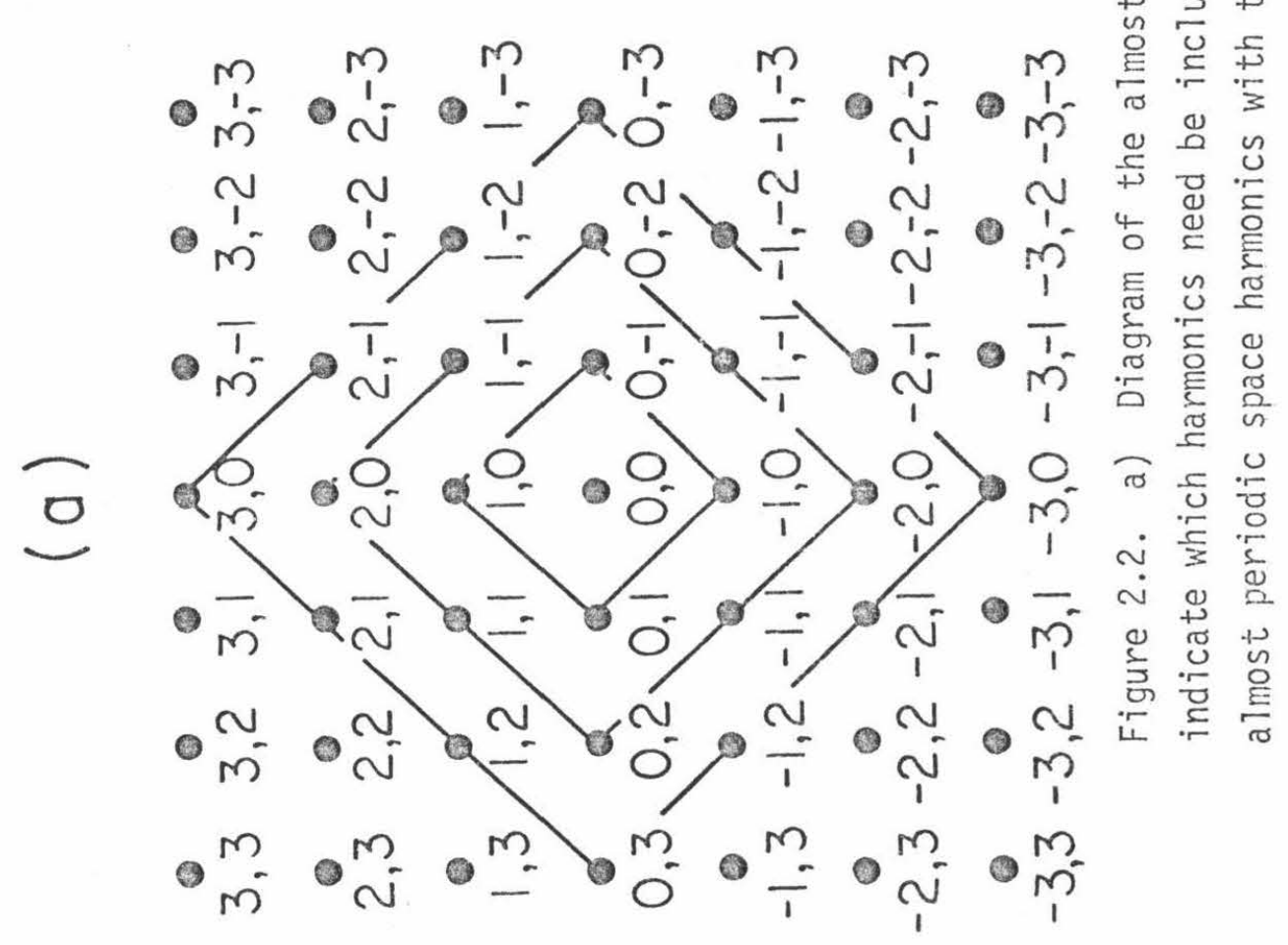


taken. This is illustrated in figure (2b) for the second-order mapping. The general rule for points to the right for a given order $2 \mathrm{~N}$ is to begin directly below the origin on the surface of the $2 \mathrm{~N}^{\text {th }}$ box, follow the box around to the last point on the right, drop directly below to the $(2 \mathrm{~N}-1)^{\text {th }}$ box, follow it around to the last point on the right and jump above to the $(2 \mathrm{~N}-2)^{\text {th }}$ box, continuing the procedure to the origin. Odd-order mappings and the points to the left are mapped analogously. Now that we have picked the mapping we can investigate the resulting theory. First, we wish to clear up a notational point. Abstractly, we can represent our mapping by the symbolic equation

$$
\{M, N\} \rightarrow L
$$

Alternatively, using the definition

$$
[A P]_{M, N}=e^{i\left(M \kappa_{1}+N \kappa_{2}\right) z}
$$

and

$$
[A P]_{L}=e^{i_{K} L_{L} a z}
$$

we could denote the mapping by the symbolic equation

$$
[A P]_{M, N} \rightarrow[A P]_{L}
$$

We will find the opportunity to employ notations of (30) later in this report.

We now wish to find the explicit form of the matrix $\underset{\sim}{\mathbb{D}}$ and the vector $\underset{\sim}{a}$ which appear in equation (22). Employing the mapping of equation (30), we find from (21) and (22) that for the first order 
34.

theory (first box)

$$
\begin{aligned}
& \underset{\sim}{a^{\top}}=\left[a_{01}, a_{10}, a_{00}, a_{-10}, a_{0-7}\right] \\
& \underset{\sim}{\mathrm{D}}=\left[\begin{array}{lllll}
\mathrm{D}_{01} & & n_{2 / 2} & & \\
& \mathrm{D}_{10} & n_{1 / 2} & & \\
n_{2 / 2} & n_{1 / 2} & D_{00} & n_{1 / 2} & n_{2 / 2} \\
& & n_{1 / 2} & D_{-10} & \\
& & n_{2 / 2} & & D_{0-1}
\end{array}\right] \\
& D_{N M}=1-\frac{\left(B+M k_{1}+N_{k_{2}}\right)^{2}}{k^{2} \varepsilon_{r}}
\end{aligned}
$$

Although our further investigations will almost exclusively involve only the first order, it is instructive to also look at the second

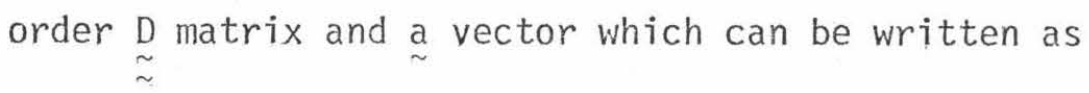

$$
\begin{aligned}
& \underset{\sim}{a^{\top}=}\left[a_{+20}, a_{+1+1}, a_{02}, a_{-11}, a_{01}, a_{10}, a_{00}, a_{-10}, a_{0-1}, a_{1-1},\right. \\
&\left.a_{0-2}, a_{-1-1}, a_{-20}\right]
\end{aligned}
$$

and $\underset{\sim}{D}$ as in figure 3.

A point noticed from the comparison of the two above orders is the striking growth of the order of $\underset{\sim}{\mathrm{D}}$ with the order of the theory. For first order, $\underset{\sim}{D}$ is five by five but in the second order $\underset{\sim}{\underset{\sim}{D}}$ has ballooned to thirteen by thirteen. From elementary considerations it is easy to see that for the theory of $N^{\text {th }}$ order, the order of $\underset{\sim}{D}$ (denoted 
35.

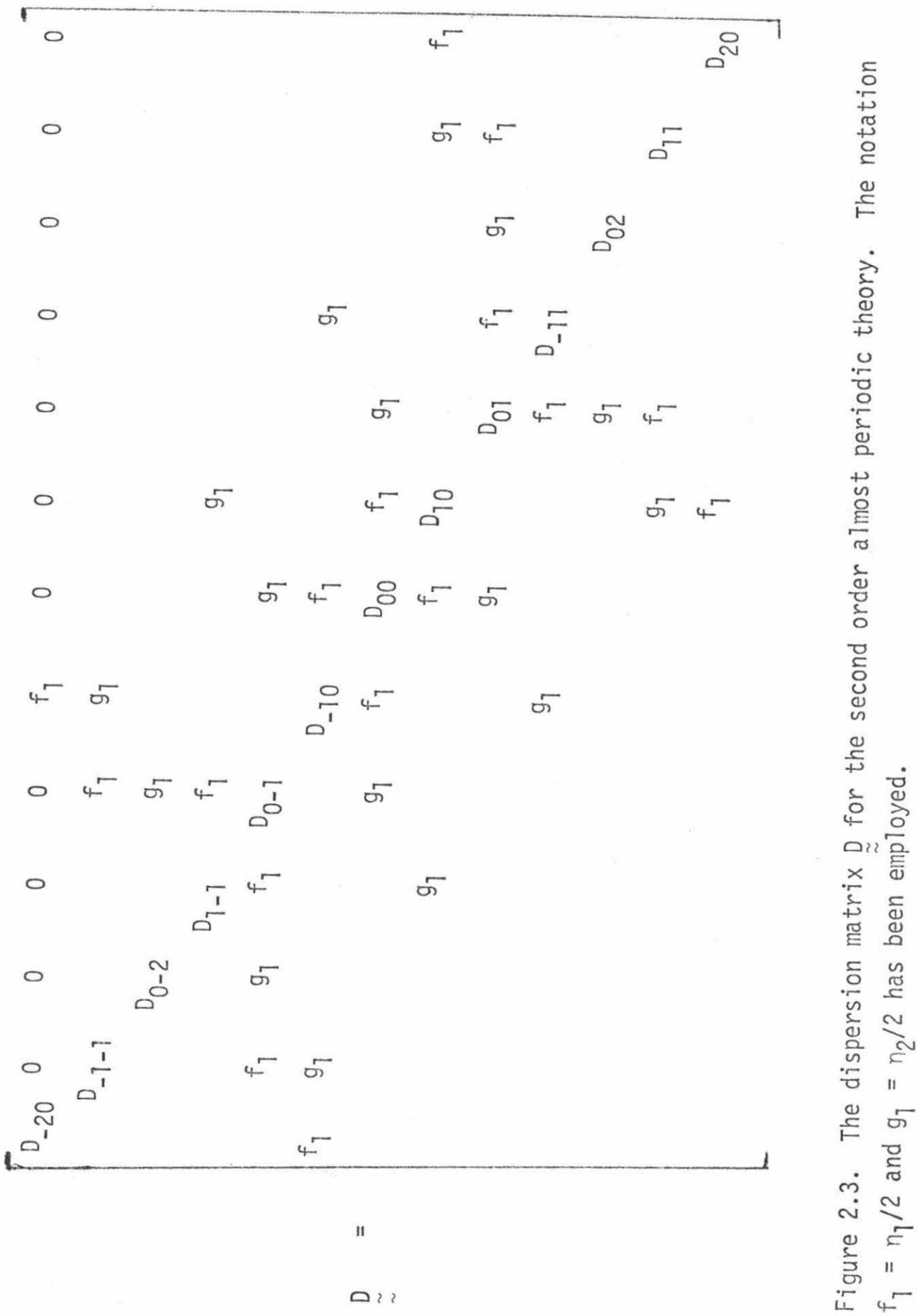


O(D)) will be given by

$$
O(D)=1+2 N(N+1)
$$

A second intereșting point can be noticed by comparing the first-order case (31b) with the fiye-by-five, two-harmonic, periodic Floquet matrix which can be written as

$$
\underset{\sim}{\sim}=\left[\begin{array}{ccccc}
D_{2} & f_{1} & f_{2} & & \\
f_{1} & D_{1} & f_{1} & f_{2} & \\
f_{2} & f_{1} & D_{0} & f_{1} & f_{2} \\
& f_{2} & f_{1} & D_{-1} & f_{1} \\
& & f_{2} & f_{1} & D_{-2}
\end{array}\right]
$$

Immediately, it is seen that the periodic $\underset{\sim}{D}$ matrix is a fuller matrix. There is physical content in this fact. Were we to write the equations represented by (22) and $\underset{\sim}{\sim}$ given by (34), we would find a coupling scheme represented by the following picture

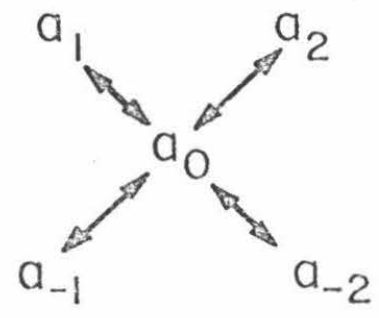

(a)

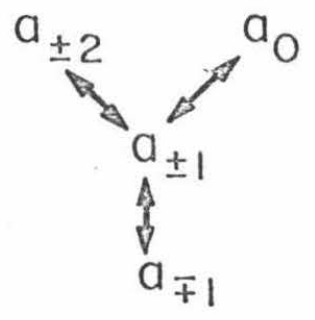

(b)

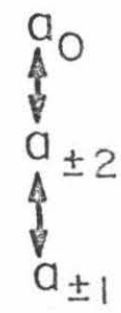

The double-arrowed 1 ine represents that the connected $a_{N}$ 's appear somewhere in the system in the same equation. The almost periodic 
coupling scheme which results from equations (22) and (37) has representation
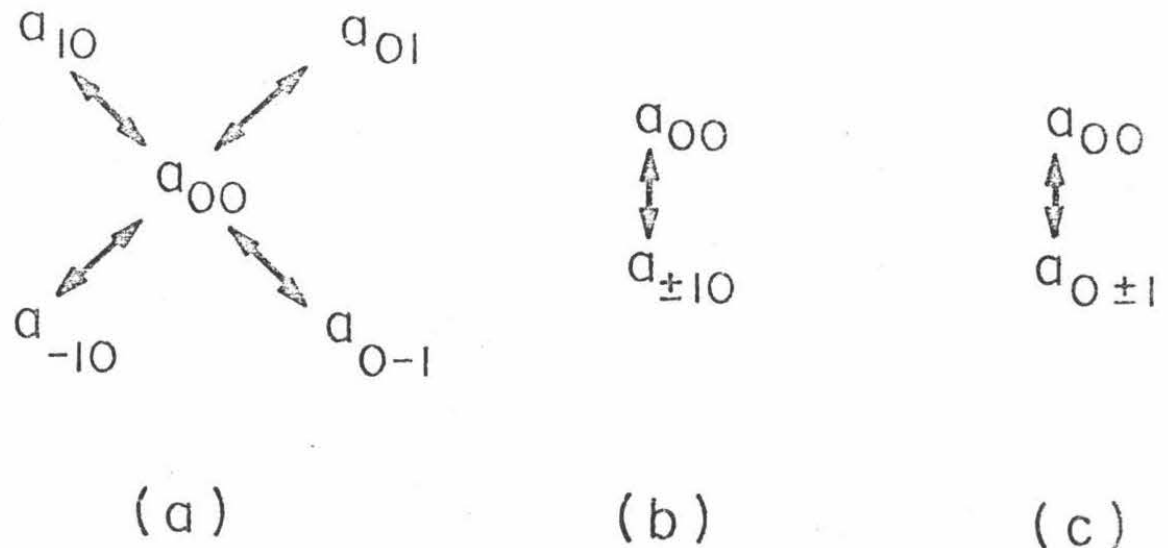

(b)

(c)

The conclusion is that the precision of the phase relation of the periodic medium leads to the richer coupling scheme of (35). In the almost periodic case, (36b) and (36c) almost give the impression that the perturbations represented by the two n's act independentiy, whereas (35b) indicates this is not so in the periodic case. We will return to this point in chapters III and IV and delve more deeply into the physical consequences.

At an earlier point we became somewhat perplexed at the lack of a Hill's determinant theory for the almost periodic solution. At this point we will try to allay some of this apprehension by considering the limiting case of smal1 perturbations. First, let us consider the case of no perturbation at a11, i.e.,

$$
n_{1}=n_{2}=0
$$

The resulting $D$ matrix of (31) becomes diagonal and has determinant 


$$
\operatorname{det} \underset{\sim}{\sim} \underset{\sim}{\sim} \prod_{M=-\infty}^{\infty} \prod_{N=-\infty}^{\infty} 1-\frac{\left(\beta+N \kappa_{1}+M k_{2}\right)^{2}}{k^{2} \varepsilon_{r}}
$$

We note that the product being over al1 $\mathrm{N}$ and $\mathrm{M}$ has terms symetrically in plus and minus $M$ and $N$. We use this fact to simplify the product, combining the term in $M$ and $N$ with the term in $-M$ and $-N$. We find (after some manipulation)

$$
\begin{aligned}
& \left\{1-\frac{\left(\beta+N \kappa_{1}+M k_{2}\right)^{2}}{k^{2} \varepsilon_{r}}\right\}\left\{1-\frac{\left(\beta-N \kappa_{1}-M k_{2}\right)^{2}}{k^{2} \varepsilon_{r}}\right\}= \\
& \left\{\frac{\beta^{2}}{k^{2} \varepsilon_{r}}-\left(1+\frac{N \kappa_{1}+M \kappa_{2}}{k \sqrt{\varepsilon_{r}}}\right)^{2}\right\}\left\{\frac{\beta^{2}}{k^{2} \varepsilon_{r}}-\left(1-\frac{N \kappa_{1}+M \kappa_{2}}{k \sqrt{\varepsilon_{r}}}\right)^{2}\right.
\end{aligned}
$$

which tells us the product can be rewritten in the form

$$
\operatorname{det} D=\prod_{M=-\infty}^{\infty} \prod_{N=-\infty}^{\infty}\left\{\frac{\beta^{2}}{k^{2} \varepsilon_{r}}-\left(1+\frac{N \kappa_{1}+M \kappa_{2}}{k \sqrt{\varepsilon_{r}}}\right)^{2}\right\} \text {. }
$$

The result is interesting for two reasons. First, as no $\beta^{\prime} s$ appear off the diagonal,(40) implies the dispersion relation can be expressed as a polynomial in $\beta^{2}$. This gives us an important part of the Hill's determinant information, namely that for a given $k$ we can always find some $\beta^{\prime}$ s, i.e., no obvious pathology occurs. Second, (40) gives us information about the form that the solution can take, i.e., it gives both the $\beta^{\prime} \mathrm{s}$ and the $\mathrm{a}_{\mathrm{NM}}{ }^{\prime} \mathrm{s}$. Consider the Brillouin (dispersion) diagram (to be discussed in detail in chapter III) of figure 4. We see the roots of (40) are straight lines extending to infinity, 
39.

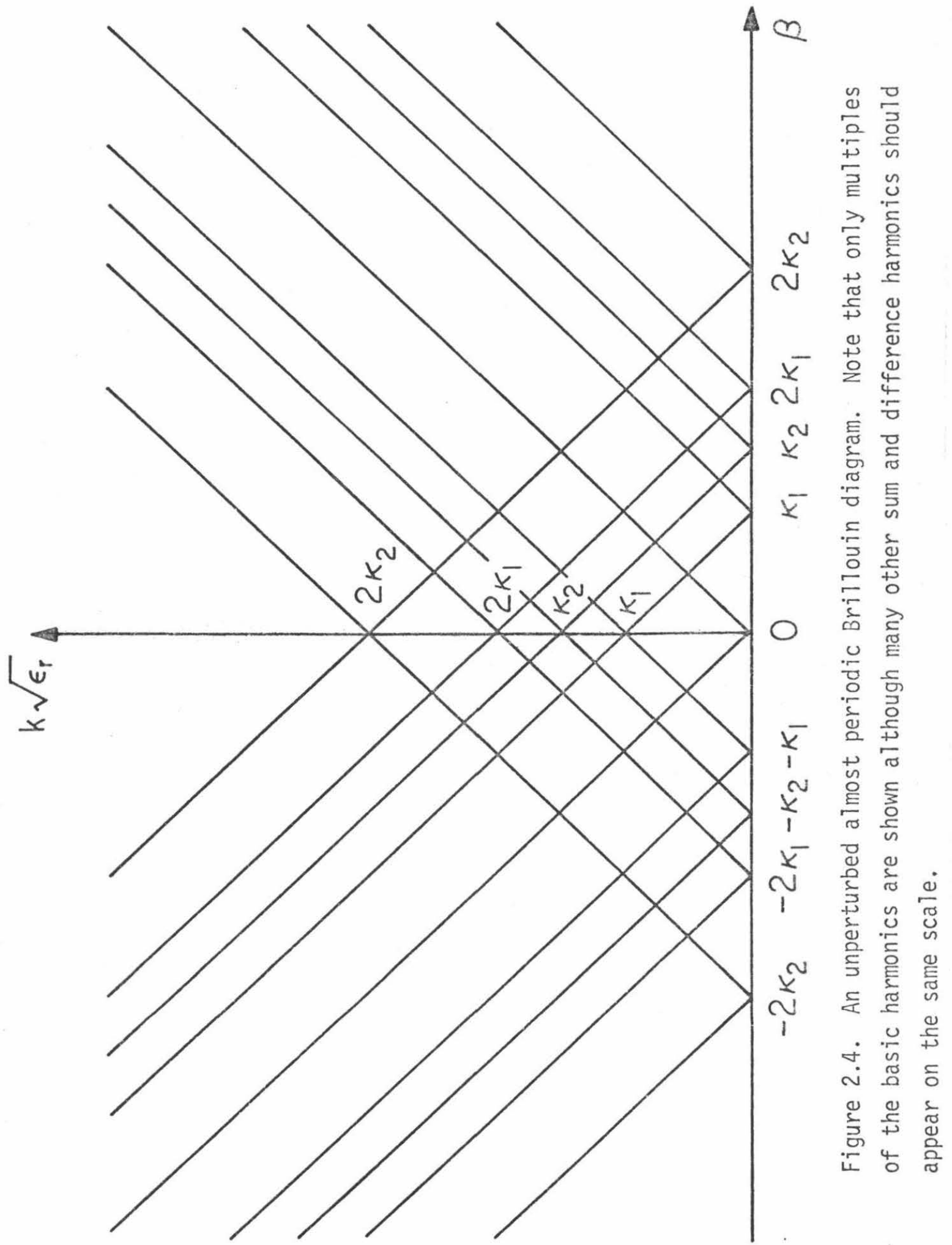


all with slopes of \pm 1 . As a determinant is a continuous function of the elements of its constituent matrix, we should think that this picture should not change too much for small perturbations. To find the ${ }^{\mathrm{NM}}$ 's for a specific line in the diagram, say the lines

$$
\beta= \pm k \sqrt{\varepsilon_{r}},
$$

we go back to equation (22) and plug in our $\beta$ value. For the periurbationless case we are considering, the equations are quite simple, i.e.,

$$
\begin{aligned}
& D_{-N} a_{-N}=0 \quad N \neq 0 \\
& D_{N} a_{N}=0 \\
& 0 \times a_{0}=0 \text { as } \quad D_{0}=0
\end{aligned}
$$

We see that we can explicitly find the one-parameter string earlier mentioned (equation 23), namely

$$
\begin{aligned}
& a_{i}=0 \quad i \neq 0 \\
& a_{0} \text { arbitrary }
\end{aligned}
$$

We see that any reasonable boundary condition on $\psi$ and hence on the $a_{N}$ 's gives us a nice bounded solution, and as the $a_{N}$ 's are also (as are the $\beta^{\prime} s$ ) continuous functions of the elements of the $\underset{\sim}{D}$ matrix, we would expect equally encouraging results for any small perturbation. It seems, in the smal1 perturbation 1 imit anyhow, that we have no need for a Hill's determinant theorem. 
Bibliography for Chapter II

1. Floquet, M. G., Ann. de 1'Ecole norm.sup. (2), XII, 47-88, (1883).

2. Whittaker, E. T. and G. N. Watson, A Course of Modern Analysis (London: Cambridge Univ. Press, Fourth Edition - 1927, reprinted - 1973).

3. The notation and form of Maxwell's equations employed in this report are those of: Papas, C. H., The Theory of Electromagnetic Propagation (San Francisco: McGraw-Hil1 Book Co., 1962).

4. Jaggard, D. L. and C. Elachi, J.A.P. 48, no. 4, 1461-1466 (1977).

5. Jaggard, D. L., App. Phys. (Springer-Verlag) 13, 185-195 (1977).

6. Papoulis, A., The Fourier Integral and It's Applications (San Francisco: McGraw-Hill Book Co., 1962).

7. Papoulis, A., Systems and Transforms with Applications in Optics (San Francisco: McGraw-Hill Book Co., 1968).

8. Goodman, J. W., Introduction to Fourier Optics (San Francisco: McGraw-Hi11 Book Co., 1968).

9. Abe1, J. M., Quart. of Appl. Math. xxviii, no. 2, 205-217 (1970).

10. Abe1, J. M. J. Math. Ana1. and Appl 36, 110-122 (1971).

11. Although the idea of using orthogonality properties of non-square integrable functions on infinite intervals is probably quite old, Norbert Wiener was certainly instrumental in systemetizing their study. A good reference is: Wiener, N., Generalized Harmonic 
Analysis-Tauberian Theorems (Cambridge: M.I.T. Press, 1964).

12. That this statement is true is evident from the Fredholm alternative theorem as applied to linear systems of algebraic equations. If we substitute a characteristic value of multiplicity one into $\underset{\sim}{\mathrm{D}}$, we can always obtain the solutions as a string of one free parameter. A method for solving the corresponding boundary value problem is discussed in the first section of chapter IV of this report.

13. A7though the form of this matrix is well known in the itterature and easily derivable, the conventions and notation employed in this report agree most closely with those of the PhD thesis of Dwight L. Jaggard. The reference is: Jaggard, D. L., Antenna Lab Report No. 75, C.I.T. (August 1976).

14. Hi11, G. W., Acta Math. viii, 1-36 (1886).

15. These other values are just simple poles and are counteracted by the multiplicative sine function. Check reference (2), pp. 415417.

16. See any reference on solid state physics, for instance: Kittel, C., Introduction to Solid State Physics (New York: John Wiley \& Sons, 1976). 
Bri1louin Diagrams in Almost Periodic Media

In the present chapter we investigate the properties of waves propagating in almost periodic media. As in the preceding and the following chapters, we borrow heavily in our attempt to implement the new theory on the well-known periodic theory and the techniques developed to implement the periodic theory. As a first cut in attempting to understand almost periodic phenomena, we concentrate on the inhomogeneityinduced dispersion of the medium. In studying the properties of the dispersion relation we employ dispersion diagrams. We shall call these diagrams Brillouin diagrams, as dispersion diagrams in periodic media are usually called Brillouin diagrams. We feel the Brillouin diagram is an effective artifice to employ in the attempt to gain physical insight into the medium's properties as pictorial information is rather easy to digest. We begin this chapter with two review sections. The first is devoted to review of the use of dispersion relations and diagrams. We feel its inclusion is warranted on the bas is that the development introduces the ensuing notation as well as providing an exposition of the concepts most useful in the following development. The second is devoted to a review of the use of the Brillouin diagram in periodic media. This section serves to highlight the techniques we are later to generalize, and gives us historical perspective.

\section{A) Basic Concepts}

To begin, we simply define the phase and group velocities of the wave, concepts so aptly treated elsewhere ${ }^{1}$. For a component of the 
electric field which can be written in the form

$$
\psi=e^{i k z-i \omega t}
$$

we find that

$$
v_{p}=v_{\text {phase }}=\frac{\omega}{k} \quad v_{g}=v_{\text {group }}=\frac{d \omega}{d k}
$$

Our Brillouin diagram will always be displayed in terms of quantities which exist in absence of modulation versus quantities that actually exist inside the medium. Using the superscript $u$ to denote unperturbed and the superscript $p$ to denote perturbed, we therefore write

$$
\begin{aligned}
& \psi^{u}=e^{i k_{0} \sqrt{\varepsilon_{r}}-i \omega t} \\
& \psi^{p}=e^{i \beta z-i \omega t}
\end{aligned}
$$

with the subsequent identifications that

$$
\begin{array}{ll}
v_{p}^{u}=\frac{c}{\sqrt{\varepsilon_{r}}} & v_{p}^{p}=\frac{\omega}{\beta} \\
v_{g}^{u}=\frac{d \omega}{d\left(k \sqrt{\varepsilon_{r}}\right)} & v_{g}^{p}=\frac{d \omega}{d \beta}
\end{array}
$$

As we are interested oriTy in variations with respect to the unpertirbed medium, we rurther make the identifications that

$$
v_{p}^{r}=\frac{v_{p}^{p}}{v_{p}^{u}}=\frac{k \sqrt{\varepsilon_{r}}}{\beta} \quad v_{g}^{r}=\frac{d\left(k \sqrt{\varepsilon_{r}}\right)}{d \beta}
$$


where $r$ denotes relative. With reference to figure 1, an archetypal Brillouin diagram, we can presently see the ease with which $v_{p}^{r}$ and $v_{g}^{r}$ can be read. The relative phase velocity of a wave is simply a quotient of $i$ ts frequency (free space wavenumber) and $i$ ts wavenumber in the medium. The relative group velocity is simply the slope of the wave's dispersion line. The quadrant of the diagram gives the direction of the phase velocity and the line's direction gives the direction of energy flow.

We now wish to consider in some depth the different regions drawn in on figure 1. In region 1, we note that both the phase and group velocity of the wave drop below the unperturbed velocities. Operation in this regime has been successfully employed in various applications such as the particle accelerator ${ }^{2,3}$ and the traveling-wave tube ${ }^{4}$. We refer to this region as the slow-wave regime. Region 3 exhibits wave solutions of greater phase velocity, yet smaller group velocity, than that of the unperturbed region and therefore dons the title of fast wave regime. Propagation in such a regime is reminiscent of plasma wave propagation and has been treated elsewhere ${ }^{5}$. The region of real interest to the present study, however, is region 2 .

In region 2 there is an imaginary group velocity, indicating that somehow the concept of group velocity needs reinterpretation. In referring to figure 2 we can see how the concept is deficient. The derivation of group velocity is predicated by our ability to construct a wave packet about the various differently wave-numbered components of electric field in our medium. In region 2 our ability to construct such packets is seriously impaired as the spatial dependence of our waves takes the 
46.

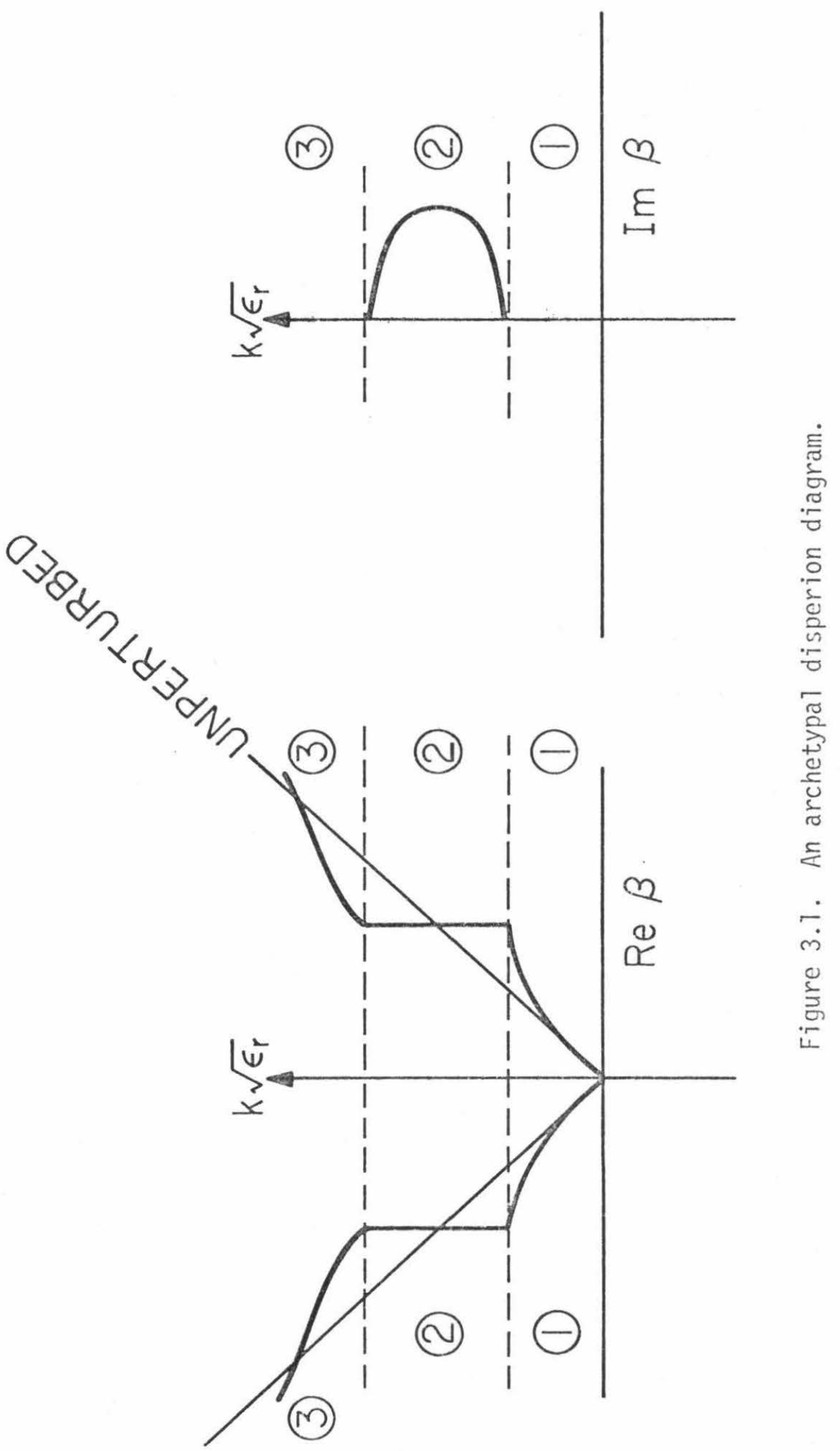



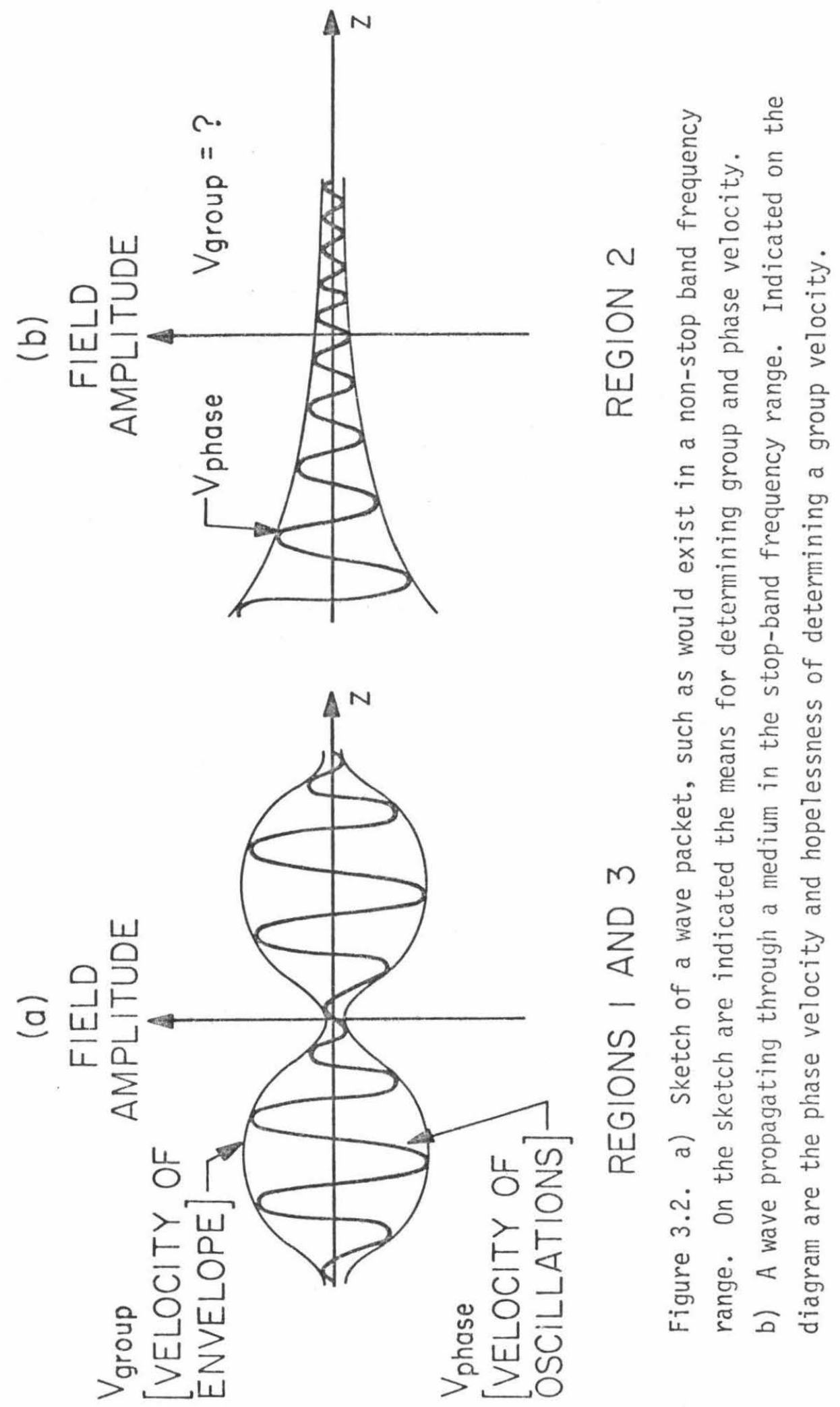
form of growing or dying exponentials. No longer can we find a specific amplitude point on a packet and follow it through the medium, as in this case the point does not move.

A slightly more physical picture of these phenomena can be constructed by considering a truncated medium, one which extends from $z=0$ to some $z=L$. The situation is depicted in figure 3. We lump the negative phase velocity waves together into a composite "backward" wave and the positive phase velocity waves analogously into a "forward" wave as has often been done in the literature ${ }^{6}$. The resulting picture shows that waves attempting to propagate into the medium with frequencies corresponding to region 2 are "redistributed in energy content" with respect to waves with frequencies outside this region. From the picture, we would expect the considered regime to be one of large reflection coefficient, as we will see it indeed is in the following chapter.

As to the terminology employed in referring to this frequency range, we feel the terms stopband or bandgap are preferable, for our purposes, to the commonly used solid-state physics term, "forbidden region" 7 . The solid-state usage is justified in the sense that thermal phonons generated in a "forbidden" energy range will not propagate through the lattice and therefore will contribute negligibly to its specific heat. However, in our case the source is external and the region is not truly forbidden, but only somewhat more reflective than at other frequencies. The distinction may be moot, but be it made. 
49.

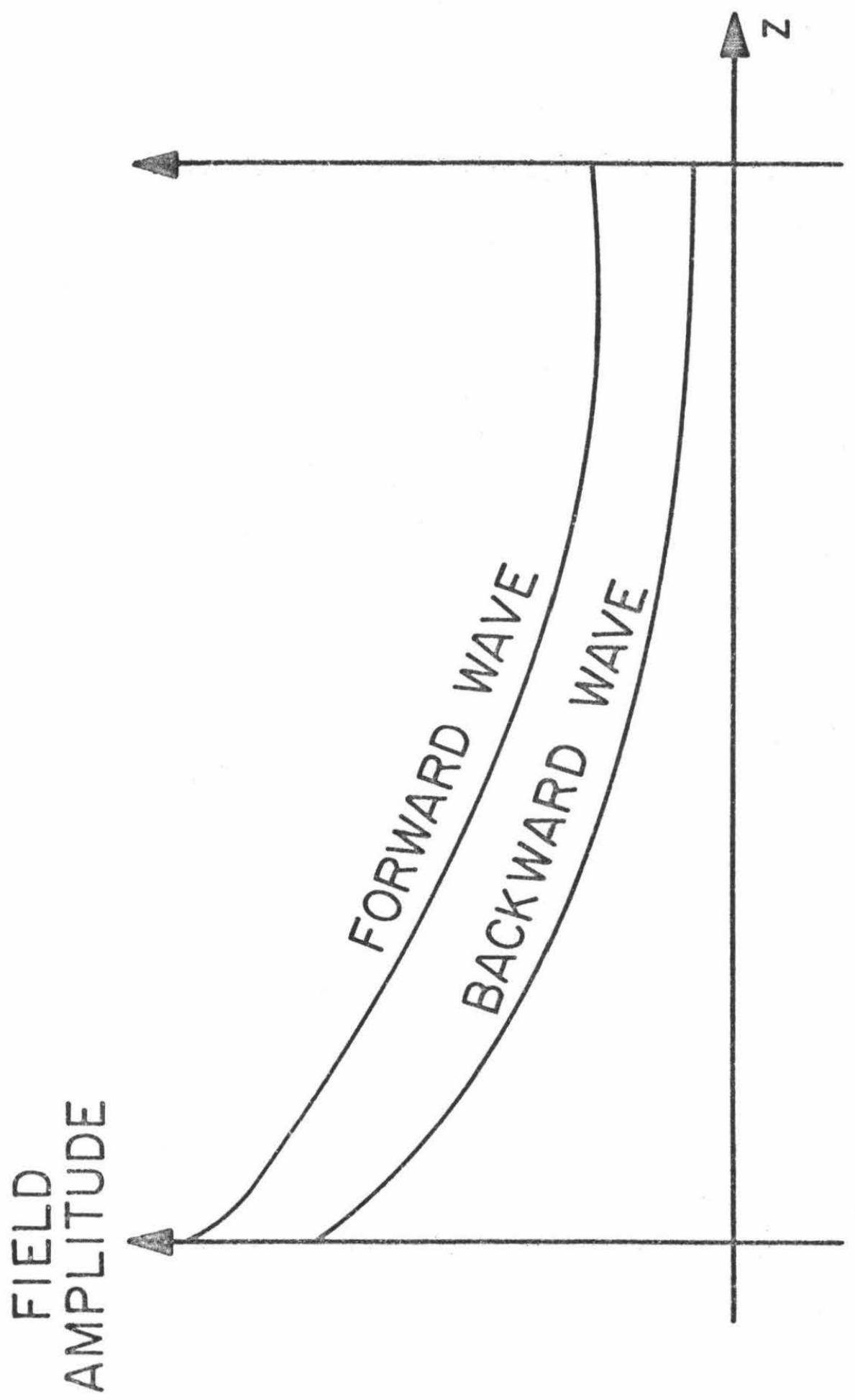

$\begin{array}{r}1 \\ 0 \\ +1 \\ \hline\end{array}$

II.

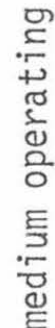

뭉

음

도

5

+5

른

告

$\frac{0}{4}$

它

4

过

宛

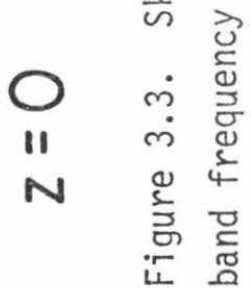


B) Brillouin Diagrams in Periodic Media--Information Contained

\section{1) Historical Perspective}

The first use of a dispersion diagram for a periodic medium in an English language journal appears to be in a 1930 work by Kronig and Penney ${ }^{8}$. Inspired by the pioneering work of Bloch ${ }^{9}$; who in late 1928 was the first to consider the problem of electron-wave propagation in a three-dimensional lattice, Kronig and Penney considered in detail the more tractable problem of one-dimensional propagation. However, between the publication of these two early works, independently Leon Brillouin ${ }^{10}$ applied dispersion diagram techniques to one-, two-, and three-dimensional lattice models and published his results in a French journal. Brillouin expanded on his technique and published works in 1932 and 1933 in which he considered problems of crystalline magnetic properties 11 and superconductivity ${ }^{12}$, respectively. The first appearance of the name Brillouin being associated with an energy band diagram was in the 1934 work by J. C. Slater ${ }^{13}$. In this work Slater terms the zones on the diagram to be Brillouin zones. This terminology caught on as is evidenced by the titles of references included in the appendix to Brillouin's definitive book ${ }^{14}$ on the topic of wave propagation in periodic structures. The book by Brillouin stems from a 1936 work 15 of his in which he first realized the great generality of periodic-media techniques. But in spite of Brillouin's realization, it appears to have been Slater who first applied the Brillouin diagram techniques, not commonly in use, to the electromagnetic wave propagation probiem in his 1948 article on linear accelerators ${ }^{16}$. The use of 
these techniques in electromagnetic problems was expanded as is evidenced by the 1959 paper of 0liner and Hesse1 17, and the term "Brillouin diagram" was certainly in standard usage by the time of the publication of the 1965 review article of Cassedy and 01iner 18 .

\section{2) Use as a Predictor}

The greatest utility we have found for the Brillouin diagram is that of a rule-of-thumb pictorial device which tells us not only which effects are important, but also gives us an expectation for the results of a physical measurement, such as that of the reflection coefficient. We will adhere to this rule-of-thumb approach in the present section by foregoing mathematical rigor in favor of physical pictures.

In considering periodic media, the first effect we should consider is that described by Bragg's 1 aw ${ }^{19}$. The familiar picture of figure 4 should make plausible the phase condition that

$$
2 \Lambda \sin \theta=n \lambda
$$

where the Bragg order $\mathrm{n}$ is an integer. In the longitudinal case which we are considering, the condition simplifies to

$$
k \sqrt{\varepsilon_{r}}=\frac{n}{2} \kappa
$$

where $k=2 \pi / \Lambda$. In cases where equation ( 7 ) is satisfied we therefore expect something to happen, the nature of which we will presently investigate.

The best vehicle with which to investigate the detailed Brillouin diagram structure would be Hill's determinant, already introduced in 
52.

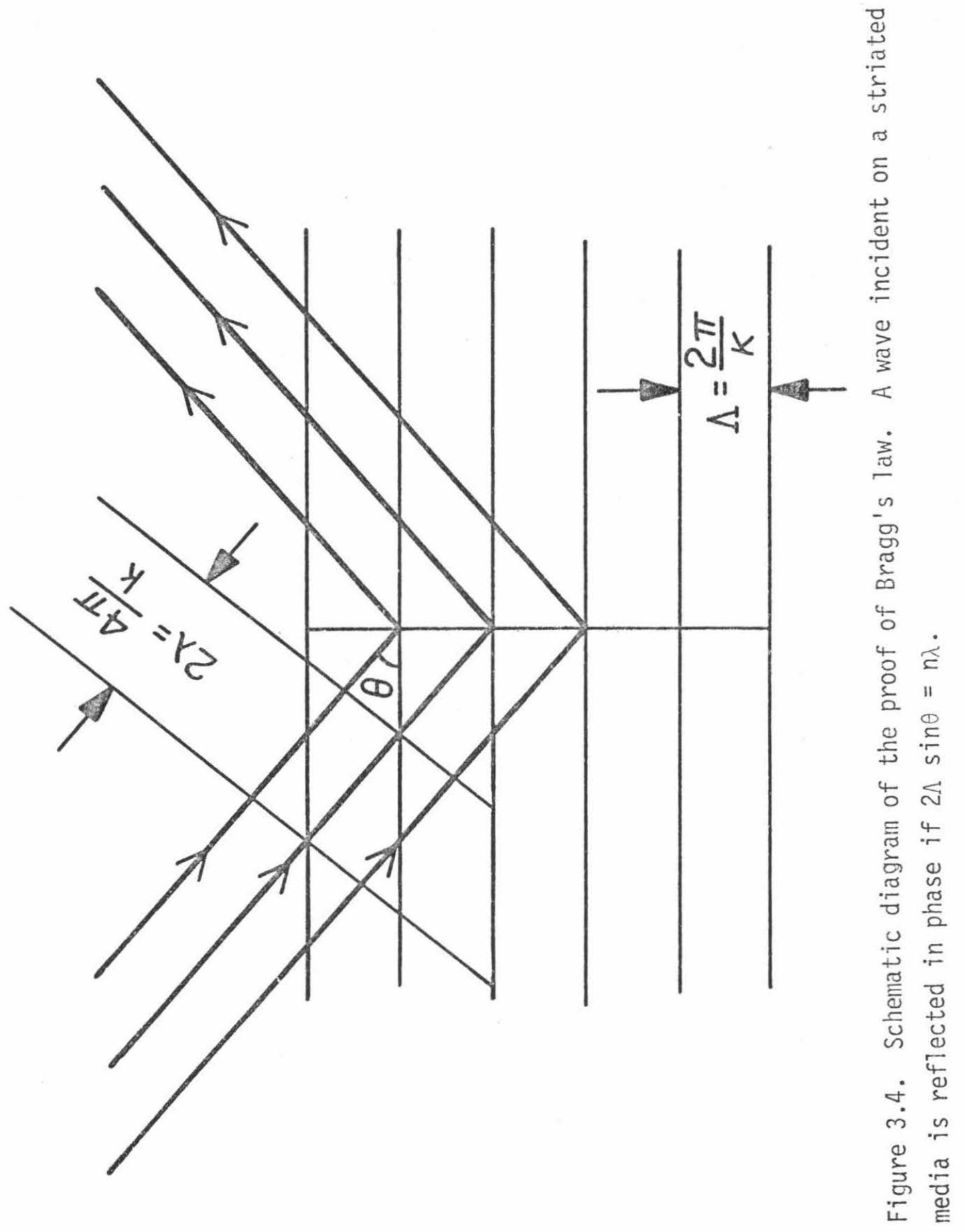


Chapter II of this report. For purposes of clear exposition we express the theory here as

$$
\sin ^{2}\left(\frac{\pi \beta}{K}\right)=A R G
$$

where

and

$$
\begin{array}{rlr}
\text { ARG } & =\Delta(0) \sin ^{2}\left(\frac{\pi k \sqrt{\varepsilon_{r}}}{k}\right), \\
\Delta(0) & =\operatorname{det}(\underset{\approx}{\Delta}, \\
\Delta_{M N} & =\left\{\begin{array}{lr}
1 & M=N \\
\frac{-k^{2} \varepsilon_{r}}{M^{2} k^{2}-k^{2} \varepsilon_{r}} f|M-N| & M \neq N
\end{array}\right\}
\end{array}
$$

$$
f_{|N-M|}=\frac{n|N-M|}{2}
$$

We wish to examine the various $\beta$ values in the proximity of the various Bragg orders to gain more insight into the nature of the solution. We first notice that in the absence of perturbation (a) $1 n_{j}$ 's equal to 0 ) the solution to (8) reduces to

$$
\beta=k \sqrt{\varepsilon_{r}}+L K \quad(L \text { any integer })
$$

We also note that this solution is noticeably changed by the inclusion of any small perturbation due to the coefficient of the off diagonal terms of $\Delta$ :

$$
\Delta_{M N}(M \neq N)=\frac{-k^{2} \varepsilon_{r}}{M^{2} k^{2}-k^{2} \varepsilon_{r}} f|M-N|
$$

which become singular for 


$$
k \sqrt{\varepsilon_{r}}=4 k \quad \text { (with reference to equation (7) } M=n / 2 \text { ) }
$$

i.e., for all even Bragg orders. Let us presently break up the situation into cases and examine the argument ARG at even and odd Bragg orders. We see from equations (7) and (8b) that for $M$ odd

$$
A R G=\left.\Delta(0)\right|_{\max } \quad(\text { maximized with respect to variations in } k)(12 a)
$$

for il even

$$
\begin{aligned}
& \text { ARG }=\lim _{\varepsilon \rightarrow 0} \Delta(0) \sin ^{2}(\pi+\varepsilon) \\
& \lim _{\varepsilon \rightarrow 0} \Delta(0) \rightarrow-\infty \\
& \lim _{\varepsilon \rightarrow 0} \sin ^{2}(\pi+\varepsilon) \rightarrow 0
\end{aligned}
$$

The situation is illustrated in figure 5 . The values of the argument can become greater than one at odd Bragg orders and less than zero at even Bragg orders, causing the value of $\beta$ to become complex.

To make the preceding argument more qualitative, consider the following expansion of the arcsin 20

$$
\begin{aligned}
& \operatorname{ARCSIN}(x+i y)=k \pi+(-)^{k} \sin ^{-1}(\nu)+(-)^{k} i \ln \left(\mu+\sqrt{\mu^{2}-1}\right) \\
& \quad k \text { integer } \\
& \mu=\frac{1}{2}\left[(x+1)^{2}+y^{2}\right]^{1 / 2}+\frac{1}{2}\left[(x-1)^{2}+y^{2}\right]^{1 / 2} \\
& \nu=\frac{1}{2}\left[(x+1)^{2}+y^{2}\right]^{1 / 2}-\frac{1}{2}\left[(x-1)^{2}+y^{2}\right]^{1 / 2}
\end{aligned}
$$


55.

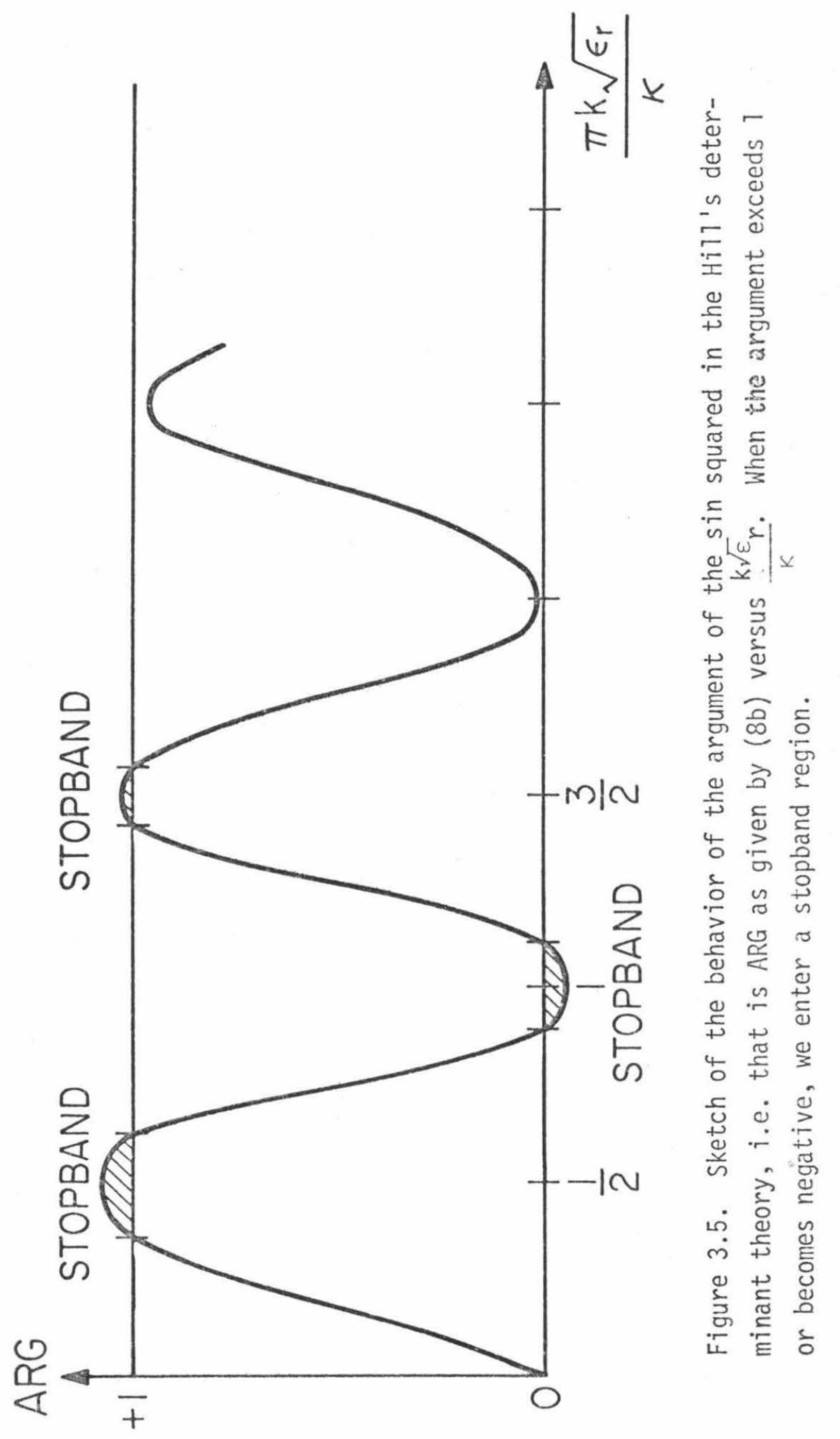


We break up the expression (13) into cases to reduce this formidable result to a more palatable form.

Case 1. When ARG $>1$, then

$$
\begin{aligned}
& \frac{\pi B}{K}=F \pi+(-)^{k} i \ln [\sqrt{A R G}+\sqrt{A R G-1}] \\
& F \text { any half-odd integer } \\
& k \text { any integer }
\end{aligned}
$$

Case 2. When $0 \leq A R G \leq 1$, then

$$
\begin{aligned}
& \frac{\pi \beta}{K}=F \pi+(-)^{k} \cos ^{-1}(A R G) \\
& F \text { any integer or half-odd integer } \\
& k \text { any integer }
\end{aligned}
$$

Case 3. When $A R G<0$, then

$$
\begin{aligned}
& \frac{\pi \beta}{K}=F \pi+(-)^{k} i \ln [\sqrt{-A R G}+\sqrt{1-A R G}] \\
& F \text { any integer } \\
& k \text { any integer }
\end{aligned}
$$

The reason for the freely specifiable integer and half-odd integer parameters is that there are an infinite number of branches of the arcsin function, causing an uncertainty as to the branch we are on. This just corresponds to the infinite number of identical branches of 
the unperturbed case. We choose to call the regime of case 1 the overflow bandgap regime, case 2 corresponds to the slow and fast wave regimes, and case 3 we denote the underflow bandgap regime.

Using equations (14-16) and figure 5 we can construct, qualitatively at least, a typical Brillouin diagram for a periodic structure as depicted in figure 6 . We see that the diagram contains a11 the regimes which we discussed in the dispersion diagram section, with the corresponding interpretations. We see, therefore, that from the pictorial representation of the dispersion relation much information about the medium's frequency dependence and reflection characteristics can be deduced. In this sense the Brillouin diagram is a guide to the prediction of a material's physical response. However, this is not the only use to which we apply the diagram.

3) The Brillouin Diagram as an Ordering Device In this section we will consider the use of the unperturbed Brillouin diagram as a guide to choosing the important terms in truncating our infinitely dimensioned dispersion relation in the case of small perturbations.

We begin by reconsidering the earlier derived periodic relation:

$$
\underset{\approx}{\mathrm{a}}=0
$$

For the moment we wish to examine the case in which we only need consider two space harmonics. In general, we will always start by considering the zeroth space harmonic, that is to say, the space harmonic corresponding to the two lines on the Brillouin diagram closest to 
58.

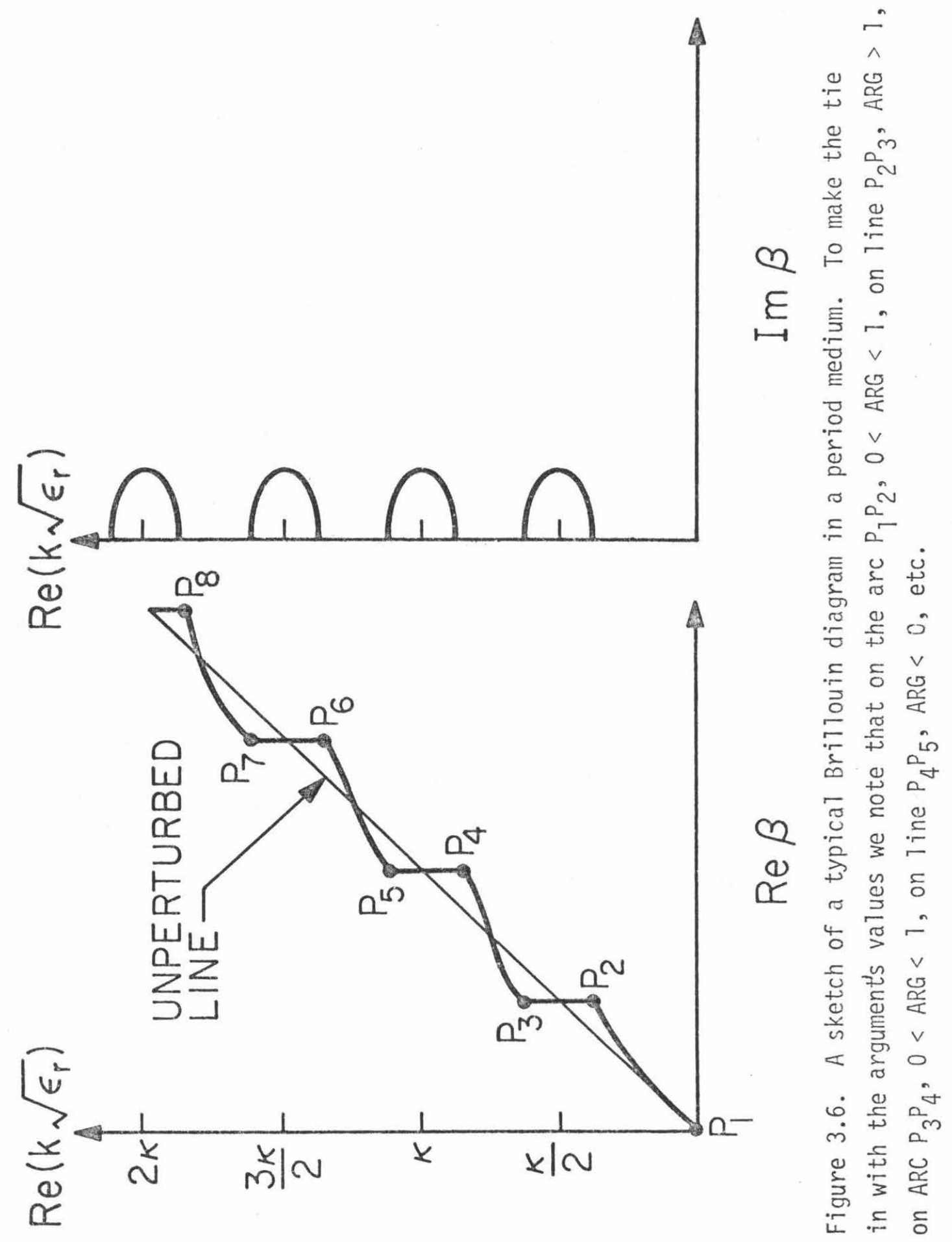


59.

those on the Brillouin diagram of the perturbationless structure with the same basic dielectric constant, $\varepsilon_{r}$. In this case we shall consider only the zeroth and the so-called minus-one harmonic (refer to figure 7. Strictly, the harmonics on the Brillouin diagram should also be superscripted by the sign of their phase velocity as in equation 17 ; each actually corresponds to two lines of the Brillouin diagram.) We continue by writing out the resulting two-by-two system obtained from equation (17) by ignoring all the zeroth and minus space harmonics

$$
\begin{aligned}
& D_{-1} a_{-1}+f_{1} a_{0}=0 \\
& f_{1} a_{-1}+D_{0} a_{0}=0
\end{aligned}
$$

and by recalling that

$$
D_{\overline{+} N}=\frac{k^{2} \varepsilon_{r}-(\beta \mp N \kappa)^{2}}{k^{2} \varepsilon_{r}} \quad(N \text { any integer) }
$$

We proceed in our argument by assuming the perturbation to be sma11 and expanding about the center of the assumed bandgaps. (This approximation is consistent with the truncation which has a ready assumed a small perturbation). Letting

$$
k \sqrt{\varepsilon_{r}}=k / 2
$$

and

$$
\begin{aligned}
& \beta=\beta_{0}+\Delta \beta \\
& \beta_{0}=\kappa / 2
\end{aligned}
$$

where $\Delta \beta$ is assumed to be sma11, we find 
60.

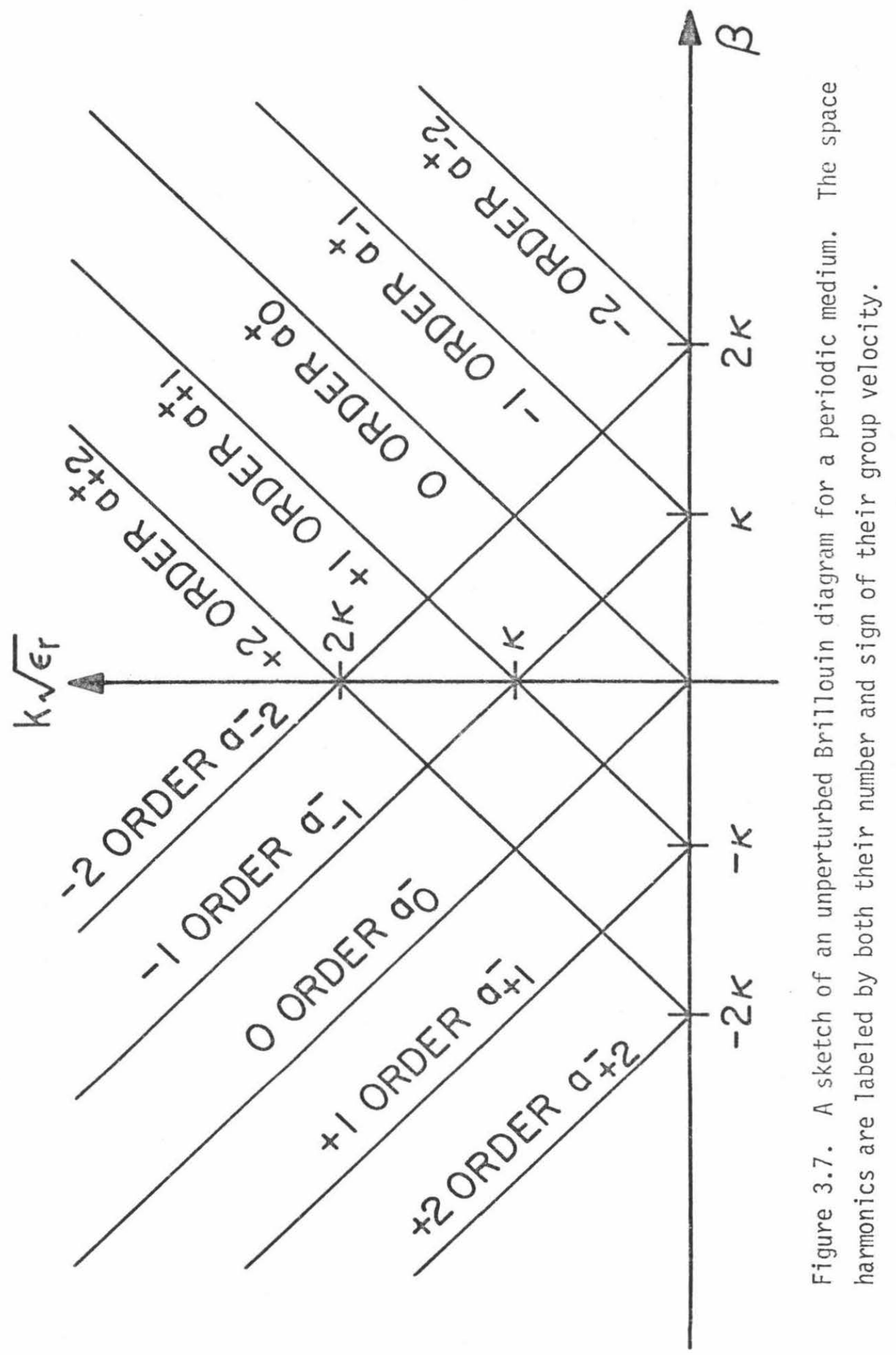


61.

$$
-D_{-1} \simeq D_{0} \simeq-\frac{4 \Delta \beta}{K} \equiv D
$$

Substituting back into equations (18) we find the ney system

$$
\begin{aligned}
\Delta \beta a_{-1} & =-x a_{0} \\
-\Delta \beta a_{0} & =-x a_{-1} \\
x & \equiv n k / 8
\end{aligned}
$$

To proceed in our interpretation we borrow from the widely used theory of coupled modes $21-30$ and consider our electric field to be comprised of a forward plus a backward traveling wave, and identify these waves as

$$
\begin{aligned}
& F=a_{0} e^{+i \Delta \beta z} \\
& B=a_{-1} e^{+i \Delta \beta z} .
\end{aligned}
$$

With these substitutions we can rewrite our system (23) in the form

$$
\begin{aligned}
F^{\prime} & =i \times B \\
-B^{\prime} & =i \times F
\end{aligned}
$$

where the primes denote differentiation with respect to $z$. We could stop at this point and attempt to determine the significance of this derivation on the Brillouin diagram, but instead we choose to push one more step forward for the sake of clarity.

We now wish to consider the system of equations complementary to (18), namely 


$$
\begin{aligned}
& D_{0} a_{0}+f_{1} a_{1}=0 \\
& f_{1} a_{0}+D_{1} a_{1}=0
\end{aligned}
$$

and make the expansion comparable to (20) and (21), but this time making the replacement

$$
\beta \rightarrow-\beta
$$

We find, analogous to (22),

$$
-D_{1} \simeq D_{0} \simeq \frac{4 \Delta \beta}{K} \equiv D
$$

resulting in the system

$$
\begin{aligned}
\Delta \beta a_{0} & =x a_{1} \\
-\Delta \beta a_{1} & =x a_{0}
\end{aligned}
$$

Now, by adding (29a) to (23a) and (29b) to (23b), we find

$$
\begin{aligned}
\Delta B\left(a_{0}+a_{-1}\right) & =\chi\left(a_{0}+a_{1}\right) \\
-\Delta B\left(a_{0}+a_{1}\right) & =x\left(a_{0}+a_{-1}\right)
\end{aligned}
$$

We could now follow the earlier line of reasoning by defining

$$
\begin{aligned}
& F=a_{0} e^{i \Delta \beta z}+a_{-1} e^{-i \Delta \beta z} \\
& B=-a_{i} e^{-i \Delta \beta z}+a_{0} e^{i \Delta \beta z}
\end{aligned}
$$

and still recover equation (25) intact. We have now rediscovered a fact already noticed in the literature 31 , namely, that it makes no difference in the expansion at the bandgap center whether we consider our 
forward (backward) going wave as comprised of a single space harmonic or a sum of space harmonics. The situation is depicted in figure 8. On occasion we shall also use the schematic representations of (8a) and (8b) which would be

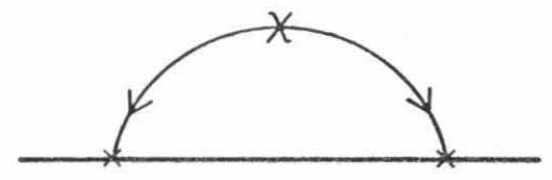

Elsewhere (Refs. 32-34) this picture is extended to include interactions at all orders and for arbitrary numbers of nonzero harmonic perturbation coefficients. We will leave the reader to check the mathematics, but include several more pictures to illustrate the technique. We feel this is a congruous approach, as the original idea of using the Brillouin diagram as an ordering device was to alleviate us from performing tedious numerical calculations until after we knew which calculations we were interested in.

In figure 9 we use the notation that

$$
X_{N}=\frac{f_{N}}{8 k} \quad(N \text { any integer })
$$

and call it the $\mathrm{n}^{\text {th }}$ order coupling coefficient. It can be

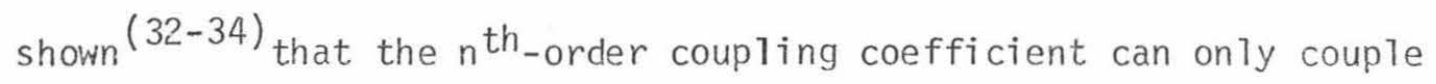
waves differing in medium wave number by $n$ units of the basic medium (or equivalently grating) frequency. This is illustrated in figure 9 where only the first and second coupling coefficient are considered to be non-zero. The figure also illustrates how the second-order coupling derives its name. We consider the coupling of the two waves at secondorder Bragg frequency to be second order if achieved by two first-order 
64.

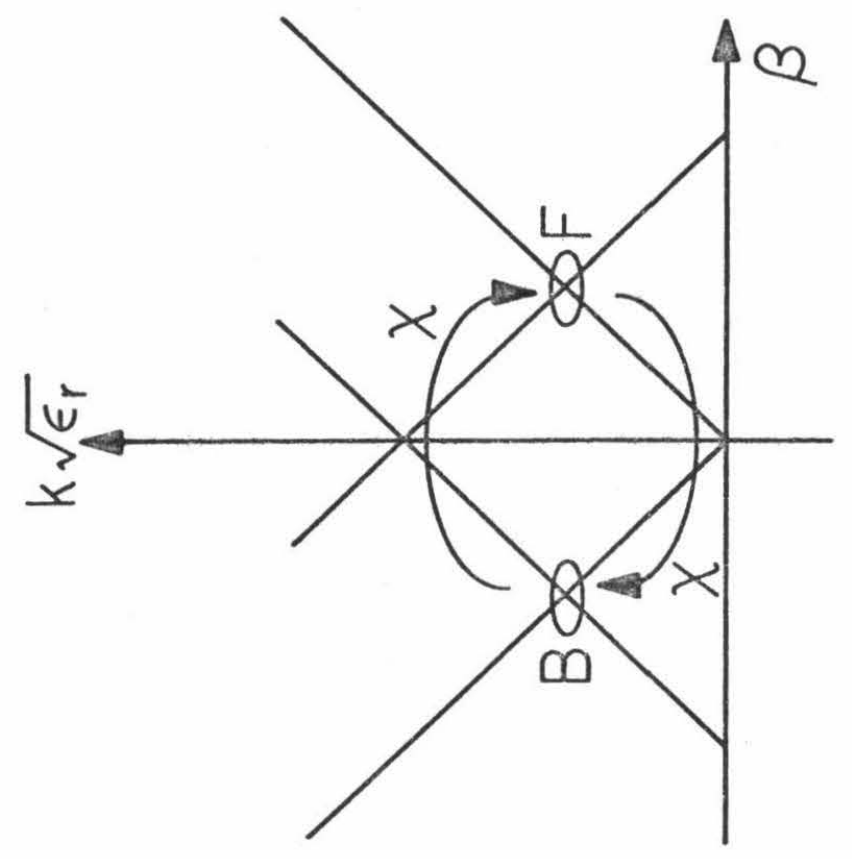

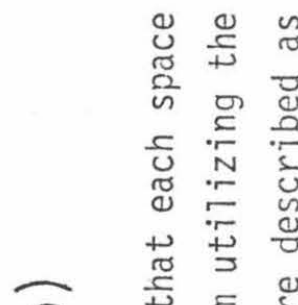

อ

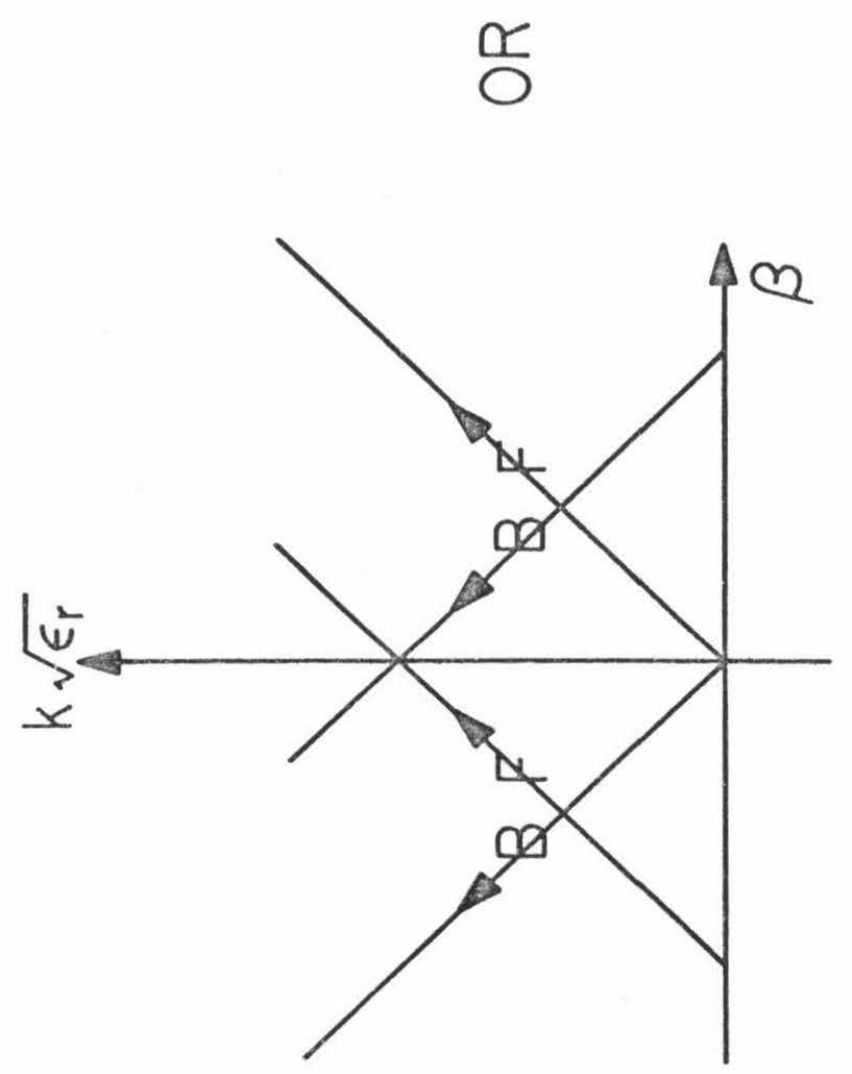

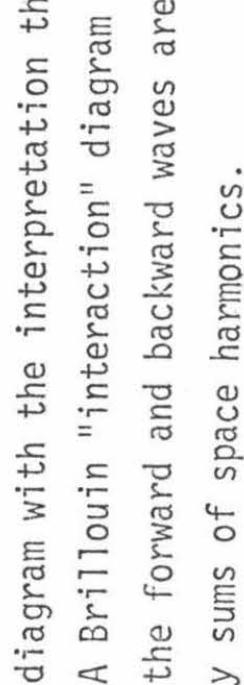

.

들

ن ป

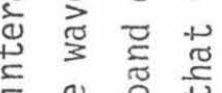

$= \pm$ के

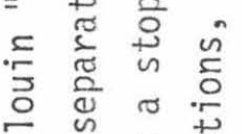

ᄃ

क n

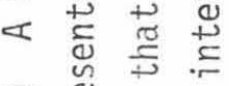

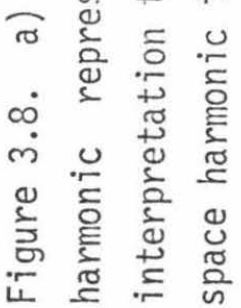


65.

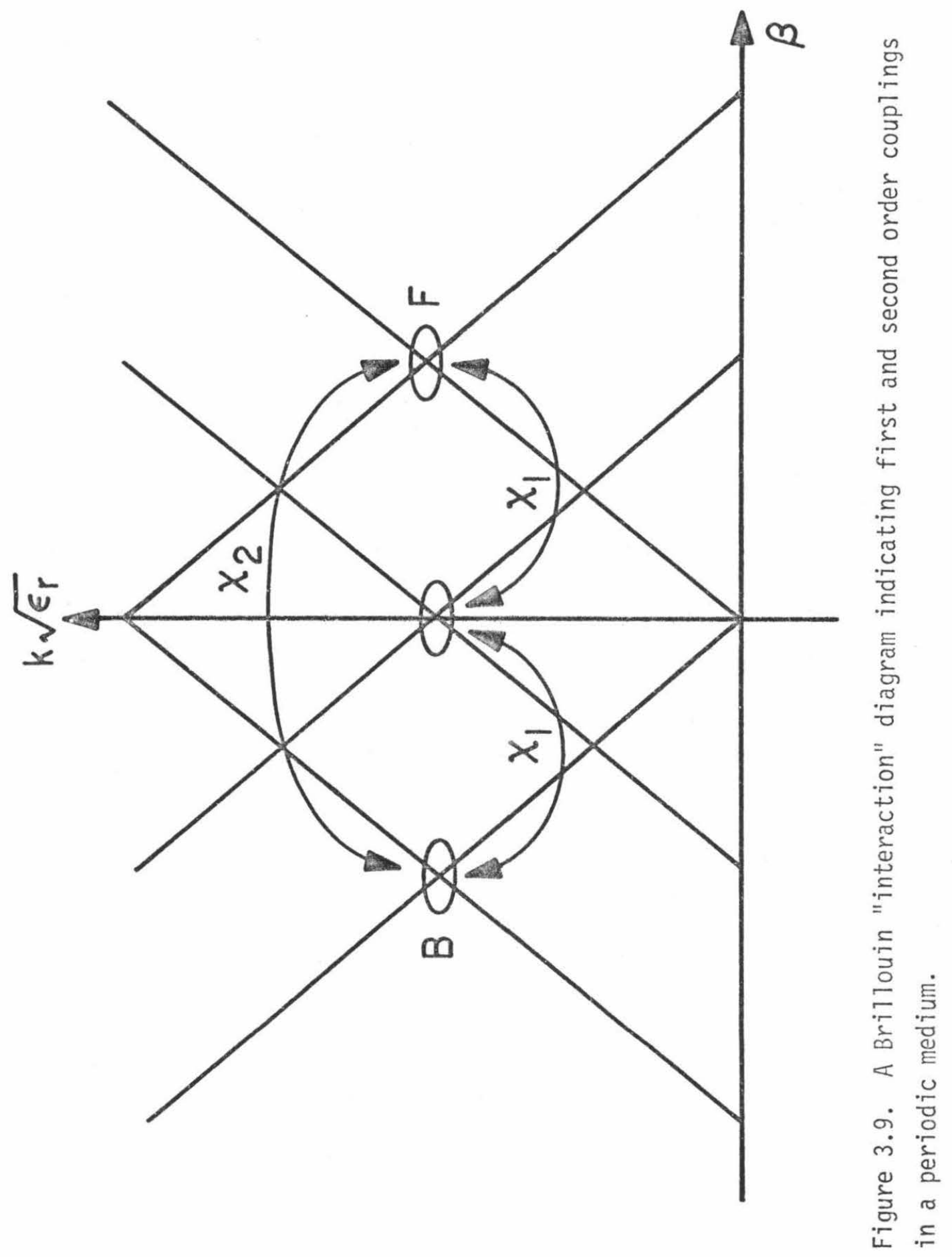


66.

interactions, or to be first order if achieved by one second order coupling. We will consider a system to be first order if it contains only the forward-to-backward wave couplings which can be achieved by a

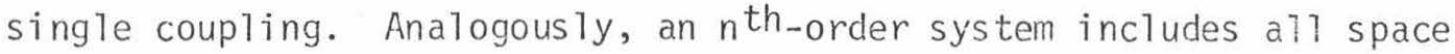
harmonics which are necessary to couple the forward-to-backward wave in n couplings or less. In general, we consider the coupling strengths to

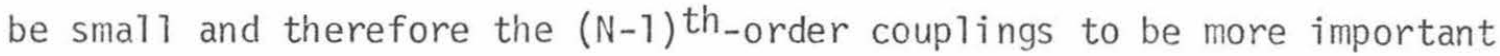
than those of the $\mathrm{N}^{\text {th }}$ order.

Figure 10 illustrates the full second-order coupling system at the second Bragg order, where only one harmonic perturbation coefficient is non-zero. In second order we notice there exist couplings which couple the forward and backward waves back to themselves. These effects are termed to be self-coupling terms and result in a movement of the bandgap center from $i$ ts unperturbed location, as is shown elsewhere ${ }^{32}$. It is important to remember that in the periodic case all bandgaps at a given frequency are identical regardless of on which space harmonic they lie. We will soon find that this is not true in the almost periodic case.

At this point we feel we have completed the discussion of the basics and will presently push on to apply the above developed techniques to the almost periodic case.

C) The Brillouin Diagram in Almost Periodic Media

Through the mapping derived in Chapter II we have seen that we can write the almost periodic dispersion relation in the form of (17). We now wish to use the Brillouin diagram to motivate our original use 
67.

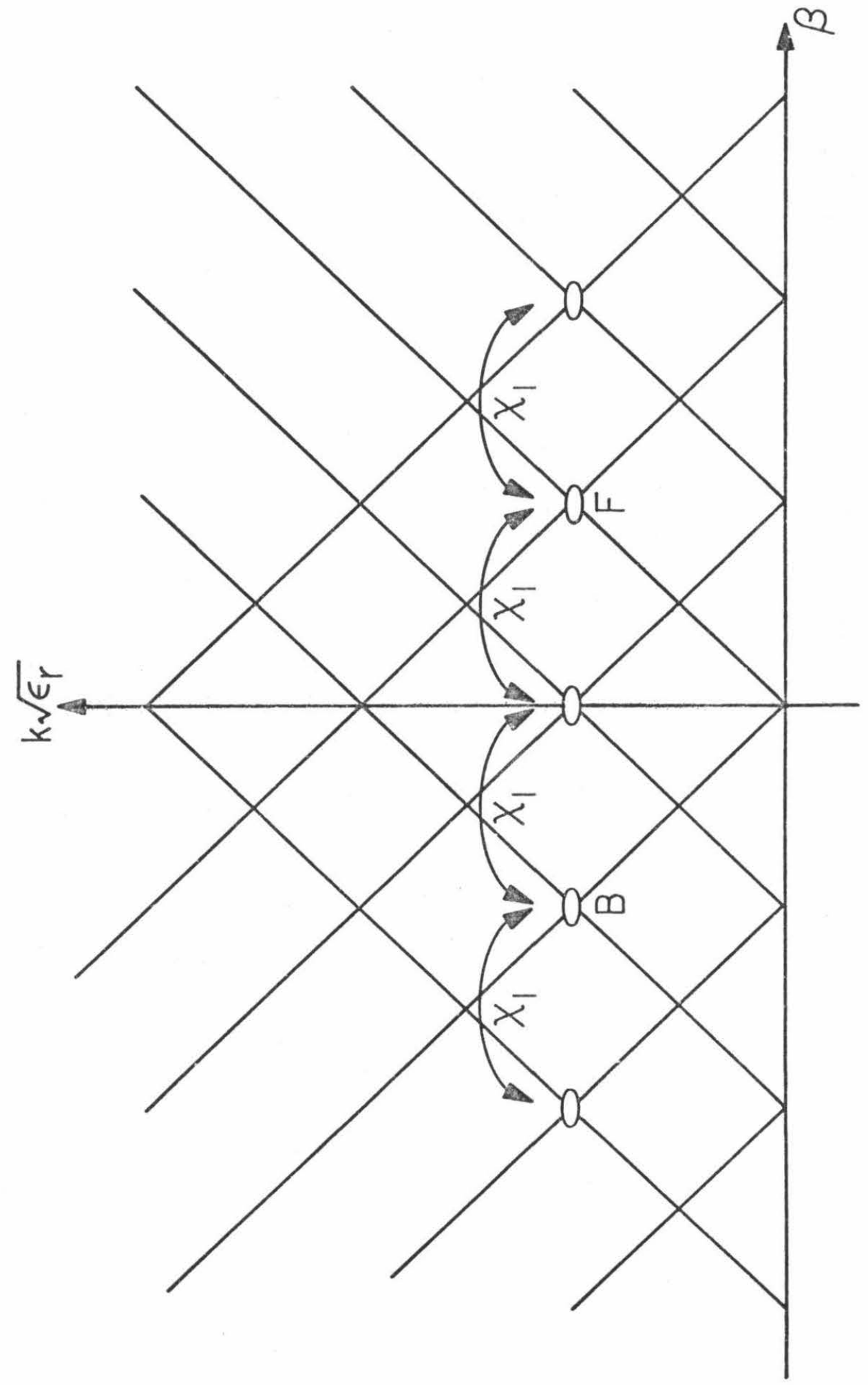

告

ปั

미

¿্․

ॠ

잉

न

ฮ ต

$+$

తु प

¿ั

.下

ह

范

$=+$

음

式

ก

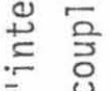

다

을 ⿺辶

$=\quad 1$

ळ 4

i $\frac{0}{+}$

लं

늠

章 
of the mapping and associated ordering definitions. Again, as in the periodic case, we consider that the externally applied wave couples completely to the zeroth-order space harmonic, which here is actually the zero-zero space harmonic.

Figure 11 illustrates the notation used for space harmonics up through the second-order theory. Also included in the diagram are all of the first-order couplings. As alluded to earlier in this report, there are but two first-order couplings, indicating, at this order of approximation, that the space harmonics do not intercouple but couple only to the zeroth-order harmonic. In this sense the theory is actually simpler than the doubly perturbed periodic theory.

One may find disturbing the fact that as the theory is taken to higher and higher order, the space harmonics do not, as in the periodic theory, show up at higher and higher wavenumber, but that the difference harmonics can become relatively dense about the origin. However, as long as the perturbation remains small, only infinitesimal amounts of energy can couple to these waves. In the absence of a Hill's determinant theorem, therefore, the almost periodic theory is limited to this small perturbation domain, as the effort necessary to generate the tremendous matrices needed in these higher order theories is prohibitive.

From figure 11 we can reconstruct the dispersion relation derived in Chapter II for the first-order theory. We rewrite that dispersion relation here as 
69.

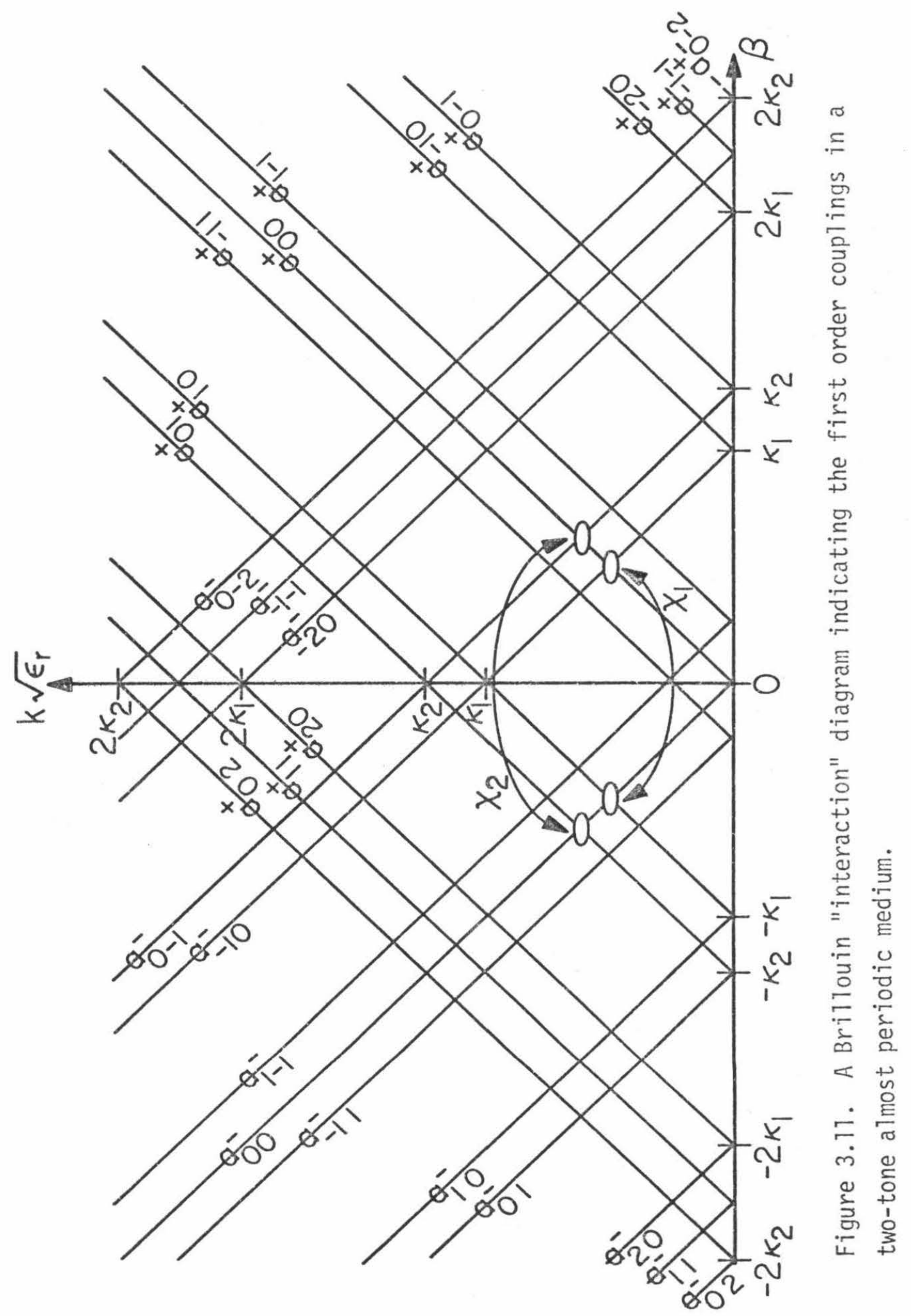


70.

$$
\begin{aligned}
& \operatorname{det}\left[\begin{array}{lllll}
D_{0+1} & & g_{1} & & \\
& D_{\dot{+} 10} & f_{1} & & \\
g_{1} & f_{1} & D_{00} & f_{1} & g_{1} \\
& & f_{1} & D_{-10} & \\
& & g_{1} & & D_{0-1}
\end{array}\right]=0 \\
& D_{i j}=1-\frac{\left(\beta+i \kappa_{1}+j \kappa_{2}\right)^{2}}{k^{2} \varepsilon_{r}} \\
& f_{1}=\frac{n_{1}}{2} \quad g_{1}=\frac{n_{2}}{2}
\end{aligned}
$$

As we have shown in Chapter II, the dispersion relation for any order is expressible as a polynomial in $\beta^{2}$. For the first-order theory we have obtained this polynomial explicitly from (34a) and found it to be expressible as

$$
P(\beta)=\sum_{N=0}^{5} \sum_{M=0}^{5} C_{N M}\left(k^{2} \varepsilon_{r}\right)^{M} \beta^{2 N}
$$

where $C$ is exhibited in figure 12.

The Hill's determinant theory and the dispersion relation of (35) were programmed on Caltech's IBM 370 for comparison. The results are exhibited in figures 13 and 14 . The test medium used had a dielectric variation of the form

$$
\varepsilon(z)=\varepsilon_{r}\left[1+\eta_{1} \cos \kappa_{1} z+\eta_{2} \cos \kappa_{2} z\right]
$$

where we took 


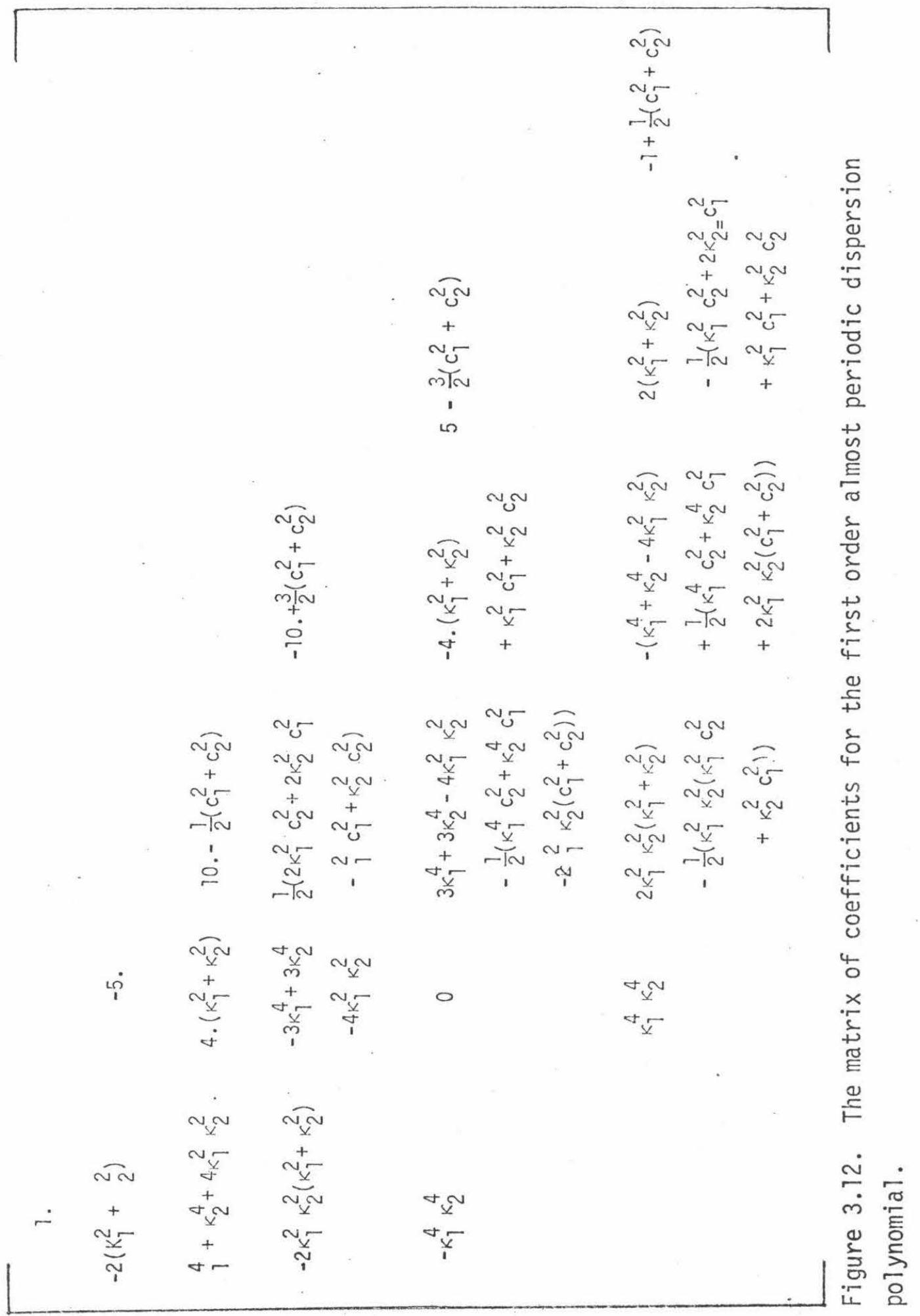




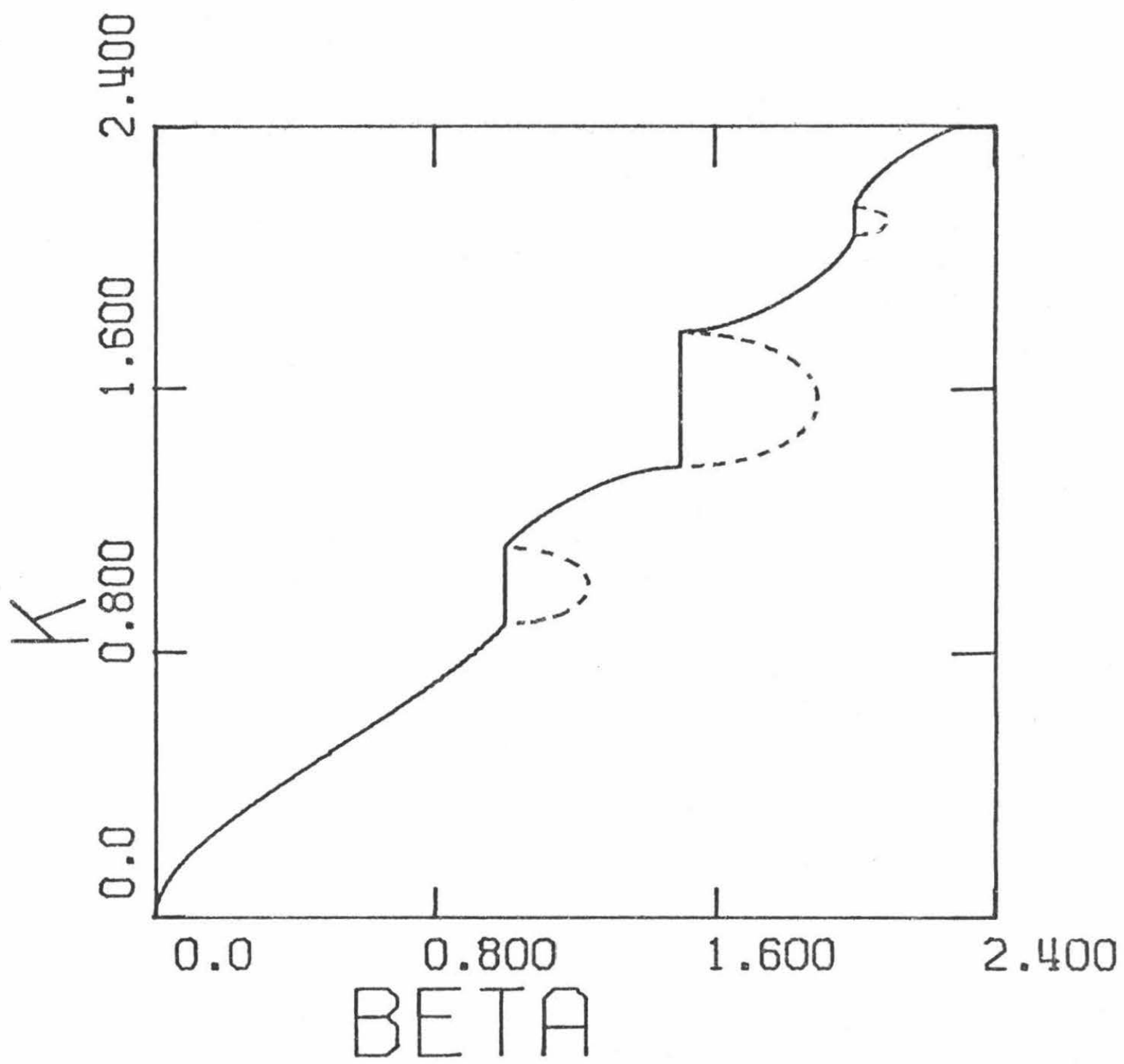

Figure 3.13. A plot of the Brillouin diagram computed from equation (8) for the periodic medium described by $\varepsilon(z)=\varepsilon_{r}[1+.5 \cos 2 z+$ $.5 \cos 3 z]$. The imaginary part of $\beta$ is the dotted 1 ine superimposed over the stopband regions. The scale of the imaginary part can be determined from

$$
\left.I_{m}(\beta)\right|_{M n V} \cong .2 \approx \frac{n N}{Q} \text { (the perturbation theory prediction) }
$$


73.

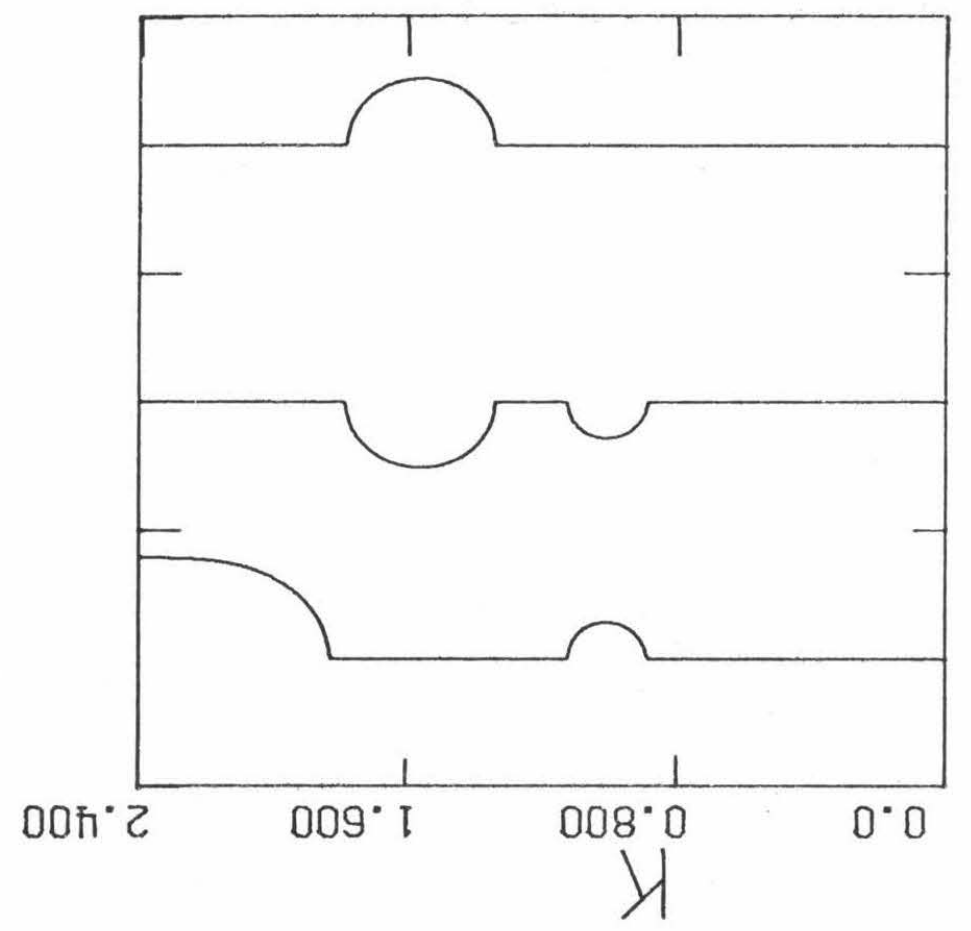

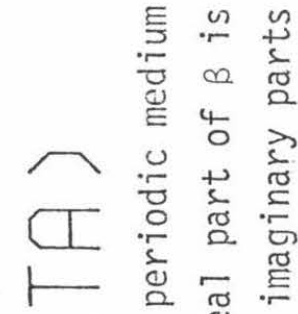

山 is

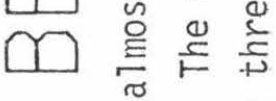

$\sum \stackrel{0}{\Sigma} \frac{0}{ \pm}$

๖े

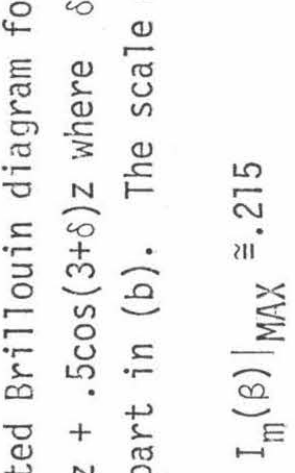

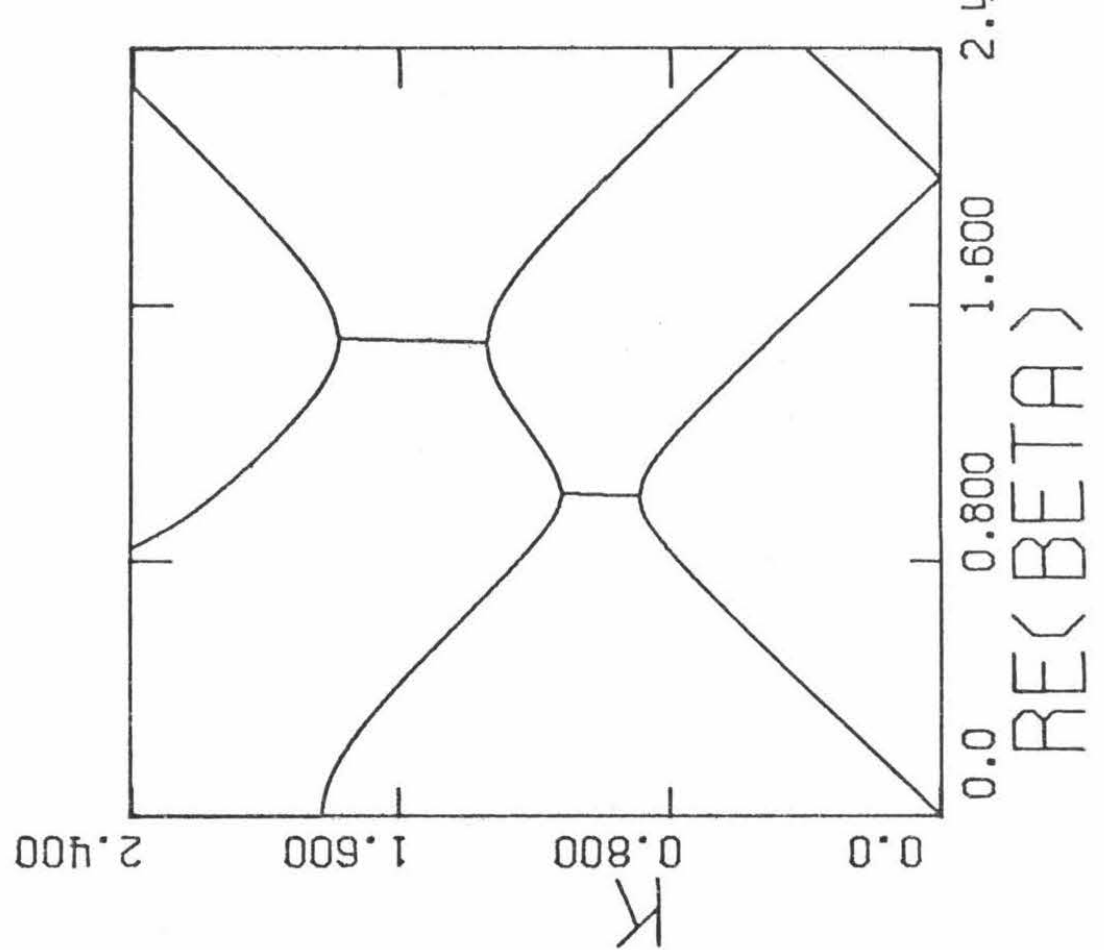

$\stackrel{ \pm}{N}$

号

U L

导 + 可

4 匚

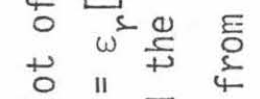

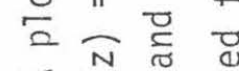

$<\frac{N}{\omega} \stackrel{d}{\circ}$

$\therefore$ ลे

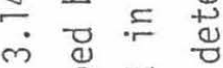

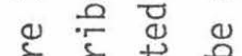

ड

䜤过 
74.

$$
k_{1}=2 \quad k_{2}=3 \quad n_{1}=.5 \quad n_{2}=.5
$$

It might be remarked that this dielectric constant (36) is periodic and does not make sense for use in an almost periodic theory. The point, however, is moot as, to computer accuracy, there is no distinction between a rational and irrational number. The two theories, however, do make the distinction automatically as the rationality or irrationality of the ratio of the perturbation frequencies is inherent in the dispersion relation derivation. Clearly, the different forms of the two theories indicate that this is certainly the case. We therefore are allowed to make a direct comparison between two media whose difference the computer cannot distinguish.

One interesting point noticeable on both plots is the greater size of the upper, with respect to the lower, bandgap. The ratio between the two sizes is seen to be close to three halves. There is a simple reason for this. As can be seen from equation (25) the important coupling parameter is always expressed per unit length. Because the wave sees more "bumps" per unit length at the higher frequency, it couples more strongly to the higher frequency perturbation linearly in a simple ratio of the frequencies. This result can be seen to be generally true, at least if the perturbations are small, from extended coupled-mode arguments presented elsewhere $32-34$.

A few words are necessary on how to read the diagrams. Figure 13 is relatively straightforward as it looks much like the simple dispersion diagram of earlier sections. Figure 14, though, is of a slightly different form. It is hard to tell a computer which branch of 
a dispersion relation to take, and therefore much easier to have it plot them al1, which is what was done. We see if we pick the most central root in figure 14 , we come very close to matching the plot in figure 13, and therefore this is the branch we want. We can match this real part with any combination of the complex conjugate imaginary parts which are plotted separately and with a different scale. All three imaginary parts are plotted and on separate lines (i.e., have different zero points). There is one minor embarrassment though, that of the large imaginary part on root 1 extending right off the diagram. This is easily explained away, however, as the theory is no longer valid in this region on this root. The polynomial only contains enough information for the first two bandgaps and the problem bandgap region Ties in the regime of the second or higher order theory.

As far as direct comparison is concerned, apart from the different plotting format, the results of the two theories appear to be almost identical for the given set of parameters. Yet we know they can't be identical everywhere, as the dispersion relations do differ. This compels us to take a closer look at the respective dispersion relations.

Let us consider the effect of phasing the two perturbations differently, that is to say, let

$$
\begin{aligned}
& f_{1} \rightarrow f_{1} e^{i \phi_{1}} \\
& f_{2} \rightarrow f_{2} e^{i \phi_{2}} \quad \text { periodic } \\
& f_{1} \rightarrow f_{1} e^{i \phi_{1}} \\
& g_{1} \rightarrow g_{1} e^{i \phi_{2}} \quad \text { almost peri }
\end{aligned}
$$


We must perform this interchange, however, in a manner which leaves the medium lossless. We find the resulting determinants can be written as

$$
\operatorname{det} D=\operatorname{det}\left[\begin{array}{ccccc}
D_{-2} & f_{1} & f_{2} & & \\
f_{1}^{*} & D_{-1} & f_{1} & f_{2} & \\
f_{2}^{*} & f_{1}^{*} & D_{0} & f_{1} & f_{2} \\
& f_{2}^{*} & f_{1}^{*} & D_{1} & f_{1} \\
& & f_{2}^{*} & f_{1}^{*} & D_{2}
\end{array}\right] \begin{gathered}
\text { for the } \\
\text { periodic }
\end{gathered}
$$

and as

$$
\operatorname{det} D=\operatorname{det}\left[\begin{array}{ccccc}
D_{0-1} & 0 & g_{1} & 0 & 0 \\
0 & D_{-10} & f_{1} & 0 & 0 \\
g_{1}^{*} & f_{1}^{*} & D_{10} & f_{1} & g_{1} \\
0 & 0 & f_{1}^{*} & D_{10} & 0 \\
0 & 0 & g_{1}^{*} & 0 & D_{01}
\end{array}\right] \quad \begin{gathered}
\text { for the almost } \\
\text { periodic } \\
\text { case }
\end{gathered}
$$

Direct evaluation of (39a) leads to a formidable algebraic equation involving all combinations and permutations of the perturbations and their conjugates. It would be too much to believe that the expression could be phase-independent and indeed it has been shown elsewhere ${ }^{32-34}$ by other techniques that it is not. The evaluation of (39b), though, is much simpler and more instructive, as can be seen from its form

$$
\begin{aligned}
D_{0-1} D_{-10} D_{00} D_{10} D_{01} & -\left|f_{1}\right|^{2} D_{01} D_{0-1}\left(D_{10}+D_{-10}\right) \\
& -\left|g_{1}\right|^{2} D_{10} D_{-10}\left(D_{01}+D_{0-1}\right)=0
\end{aligned}
$$


Equation (40) is more important for what it lacks than for what it contains, namely the lack of an explicit dependence on the phasing of the perturbations. In some sense the perturbations are independent of one another. The only way that they could interact is through matrix elements involving $D_{i j}$. However, in the scheme we have been using to analyze the interaction where attention is placed on a bandgap center, the $D_{i j}^{\prime} s$ corresponding to different perturbations show up expanded to different orders. To illustrate, consider the expansion around the lowest order where we take

$$
\begin{gathered}
k \sqrt{\varepsilon_{r}}=k_{0} \sqrt{\varepsilon_{r}}+\Delta k \simeq \frac{k_{1}}{2}+\Delta k \\
\beta=\beta_{0}+\Delta \beta \simeq \frac{{ }^{k} 1}{2}+\Delta \beta
\end{gathered}
$$

Expanding the three important D's we find

$$
\begin{aligned}
& D_{0} \sim \frac{4}{k_{1}}(\Delta k-\Delta \beta) \\
& D_{-1} \sim \frac{4}{k_{1}}(\Delta k+\Delta \beta) \\
& D_{-2} \sim \frac{4}{k_{1}}(\Delta k+\Delta \beta)-\frac{4 \Delta K}{k_{1}}
\end{aligned}
$$

where

$$
\Delta k \equiv k_{2}-k_{1}
$$

For validity of the expansion we must have

$$
\begin{aligned}
& \Delta k \ll \beta_{0}=\frac{\kappa}{2} \\
& \Delta \beta \ll \beta_{0}=\frac{k}{2}
\end{aligned}
$$


However, we have in no way specified $\Delta k$, although we can easily see that something fundamental changes when $\Delta k$ is allowed to become the same order as other parameters in the expansion. He would perhaps expect that this limit will yield part of the information we seek concerning the relation of periodic and almost periodic wave propagation, and we will therefore take up this limit in detail in the next section, where we wish to continue to consider the distinctions between the cases when the harmonic perturbations are in phase and the bandgap spacing goes to zero.

D) The $\Delta k \rightarrow 0$ Limit

1) Need for a New Perturbation Theory

The first necessity in investigating the small $\Delta k$ limit is to find exactly how small $\Delta k$ must be to enter this limit. We know that this "band spacing" variable must be on the order of the other small parameters in the calculation, namely $\Delta \mathrm{k}$ and $\Delta \beta$. However, these parameters are variable and therefore we need to know their maximum region of variation in terms of some other constant. The needed relation is easily found from equation (25). It is easily shown 32-34 that the approximate bandgap width (figure 15) is given by

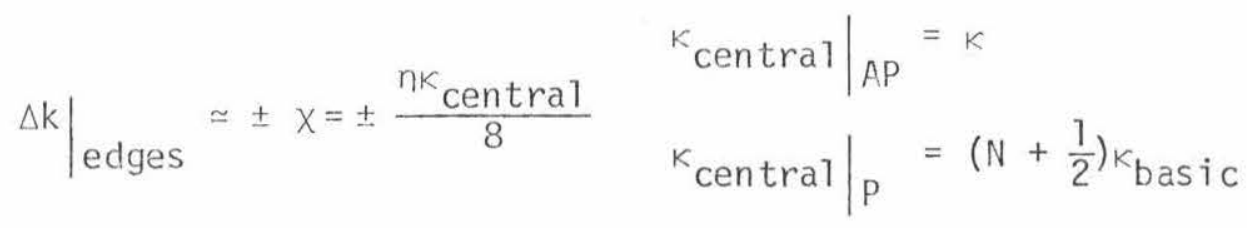

where $\kappa_{\text {central }}$ is the average of $\kappa_{1}$ and $\kappa_{2}$. It therefore follows that

$$
\Delta \kappa \simeq \frac{n \kappa \text { central }}{8}
$$




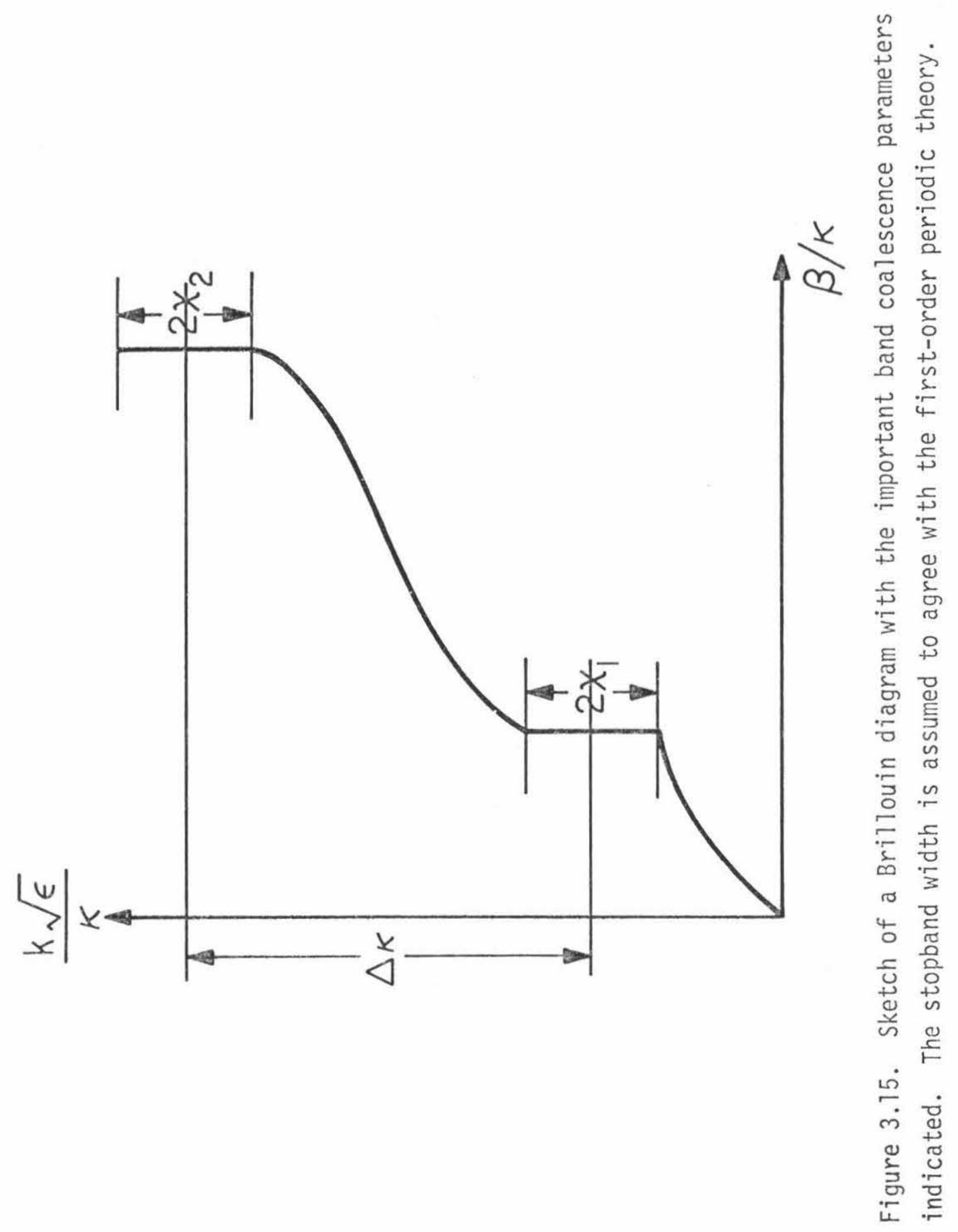


80.

As can be seen from figure 15, the limit of (45) must correspond to a "coalescing" of the two bands into one, or at least some kind of overTap.

Figures 16 and 17 are attempts to delve into the band coalescence regime employing our almost period dispersion relation program. Figure 16 just starts to enter the interesting regime as

$$
\begin{aligned}
\Delta \kappa & =.1 \\
x & =\frac{n k}{8} \sim .07
\end{aligned}
$$

Figure 17, however, al though into the band coalescence region, is somewhat disappointing, as the variation of the band structure is too rapid for our sorting routine to pick up and much interesting and valuable information is lost. In the next section we will amend this problem.

Attempts to investigate the periodic band coalescence with our present program are equally disappointing. In the periodic case we are forced to fix $\Delta k$ at unity and reach our desired limit by applying our perturbations at larger and larger multiples of the basic harmonic. But as the perturbations are placed at the $\mathrm{Nth}$ and $(\mathrm{N}+1)^{\text {th }}$ harmonic we find the size of the matrix whose determinant we must evaluate must be of order

$$
0 \underset{\approx}{\stackrel{\Delta}{\approx}} \simeq 2 N+1
$$

for any accuracy to be achieved. But (47) places a restriction on how large we can reasonably let $\mathrm{N}$ become due to the expense of obtaining the determinant of a large matrix numerically. As we have taken our 
31.

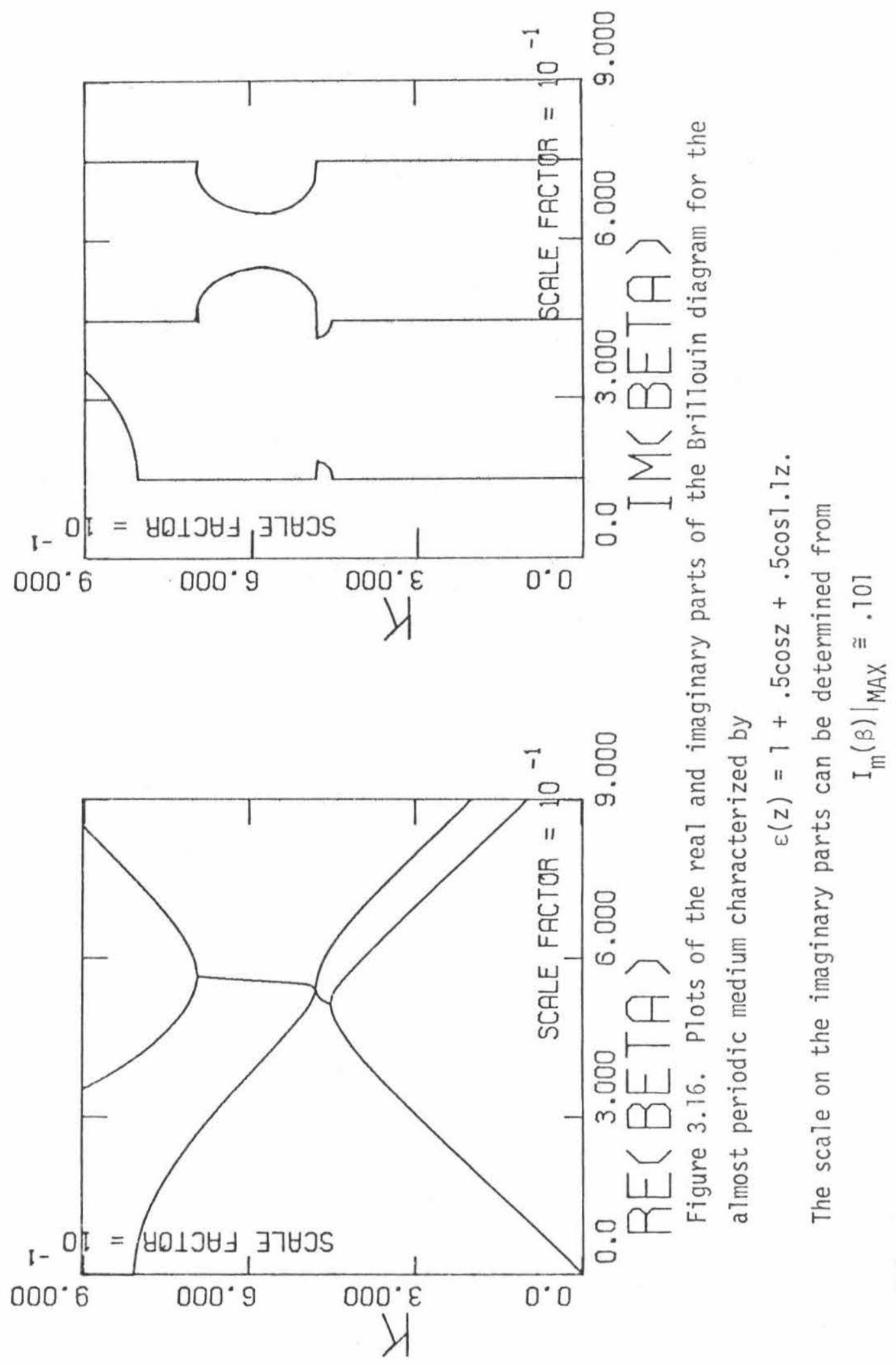




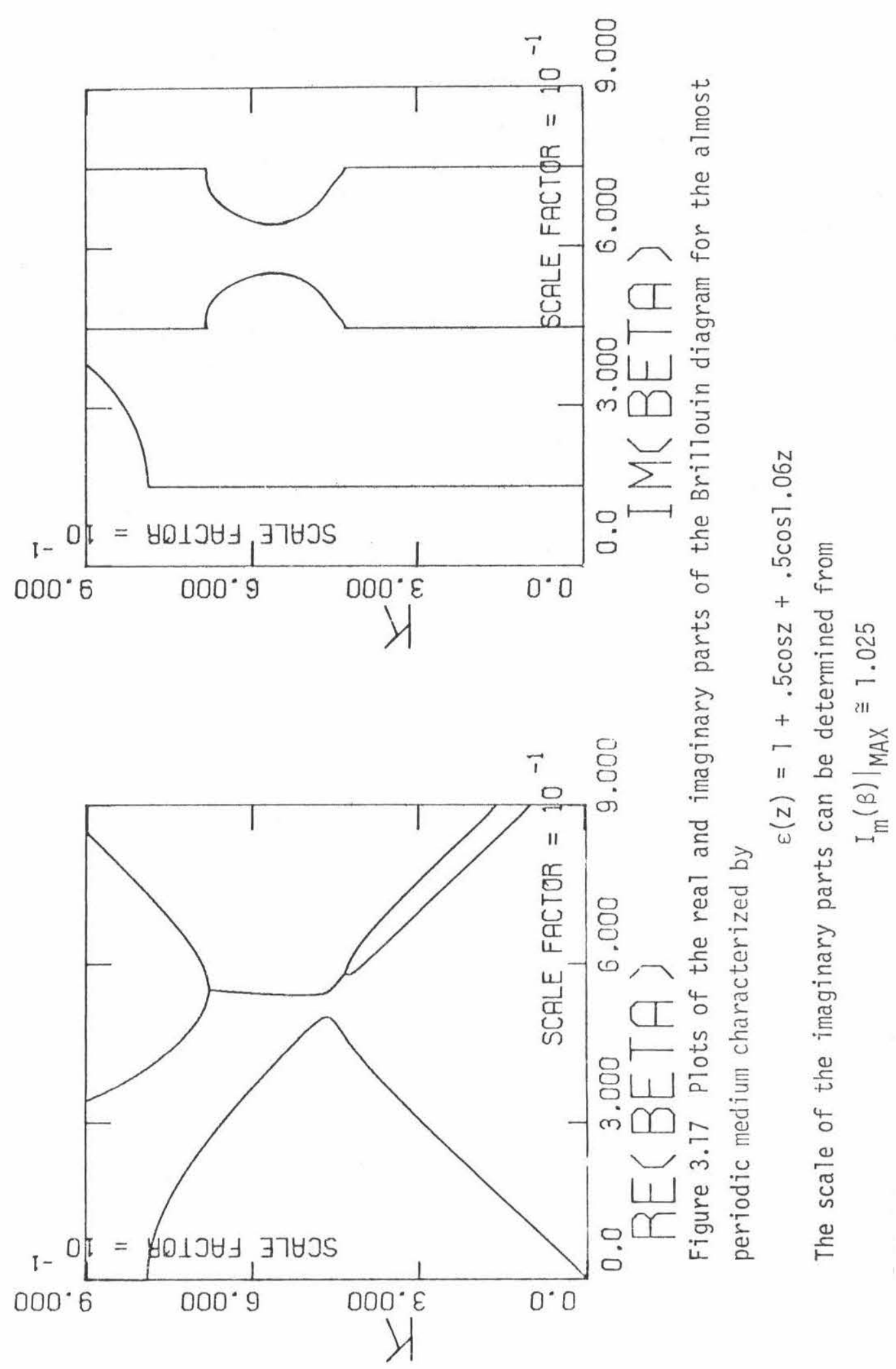


basic period to be unity we see that

$$
x=\frac{n N \kappa_{\text {basic }}}{8}=\frac{n N}{8}
$$

Taking the reasonable limit of $N=20$, we find that

$$
\eta \sim .4
$$

to achieve the band coalescence limit. Figures 18 and 19 illustrate the result for the values

$$
\begin{array}{lll}
n_{1}=.3 & n_{2}=.3 & \text { Figure } 18 \\
n_{1}=.5 & n_{2}=.5 & \text { Figure } 19
\end{array}
$$

The result is rather surprising. Instead of two bands coming together, we see many bands popping up at both super- and sub-harmonics of the expected ones. But due to the large size of the perturbations we must wonder if this effect is not really due to a breakdown of our perturbation theory instead of some more physical reason. It is now evident from figures 16-19 that an improved theory would be desirable for both the periodic and almost periodic cases, especially if one could be devised that could reach the band coalescence region without requiring a large perturbation. Such a theory is the subject of the following two sections.

2) Almost Periodic Perturbation Theory Here we wish first to perform the expansions of equation (42) in a more symmetric manner. We take the dielectric constant to be in the form

$$
\varepsilon(z)=\varepsilon_{r}\left[1+\eta_{1} \cos (\kappa-\delta) z+\eta_{2} \cos (\kappa+\delta) z\right]
$$




\section{4.}

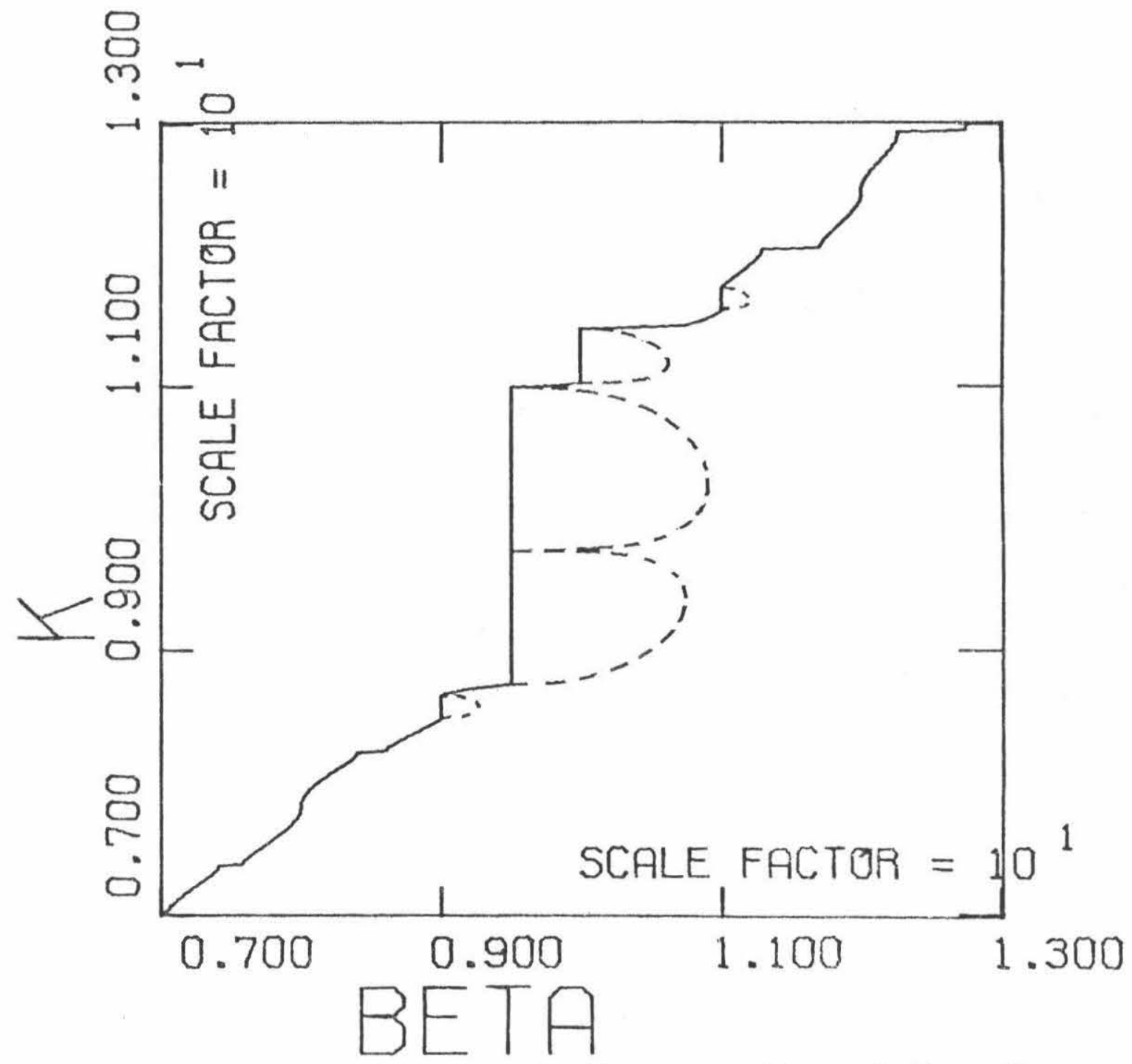

Figure 3.18. Plot of the Brillouin diagram for the periodic media characterized by

$$
\varepsilon(z)=1+.3 \cos 19 z+.3 \cos 20 z
$$

The imaginary part of $\beta$ is superimposed on top of the stopband regions. The scale of the imaginary part can be determined by noting that the maximum imaginary part is

$$
\left.I_{m}(\beta)\right|_{\text {MAX }} \cong 7 / 8
$$


35.

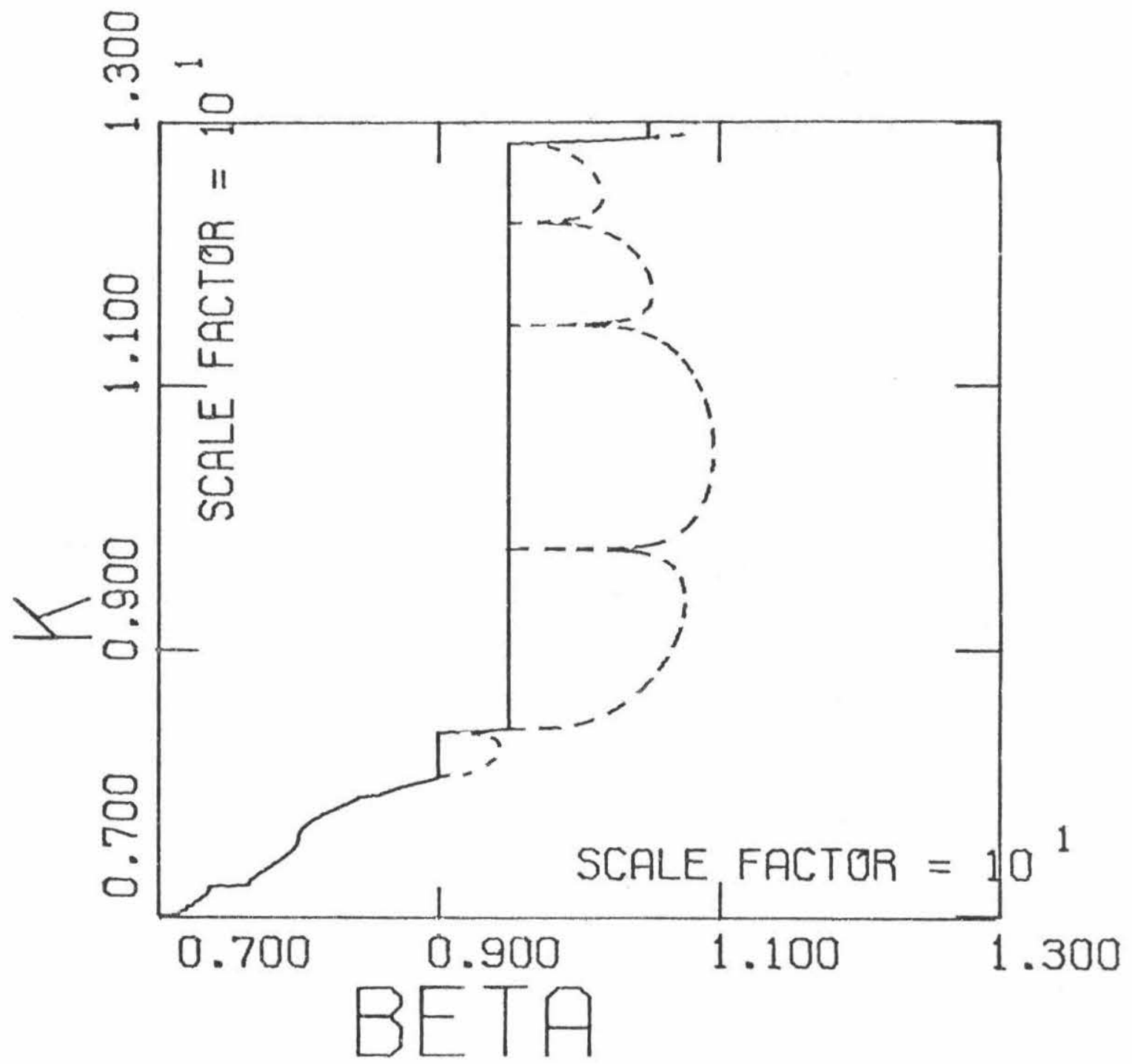

Figure 3.19. Plot of the Brillouin diagram for the periodic media characterized by

$$
\varepsilon(z)=1+.5 \cos 19 z+.5 \cos 20 z
$$

The scale of the superimposed imaginary part is determined by

$$
\left.I_{m}(\beta)\right|_{\text {MAX }} \cong 1.4
$$


where the $\delta$ and the $n^{\prime} s$ are first order quantities. We now expand the quantities

$$
\begin{gathered}
k \sqrt{\varepsilon_{r}}=k_{0} \sqrt{\varepsilon_{r}}+\Delta k \simeq \frac{k}{2}+\Delta k \\
\beta=\beta_{0}+\Delta \beta \simeq \frac{k}{2}+\Delta \beta
\end{gathered}
$$

Analogously to (42), we find

$$
\begin{aligned}
& D_{0} \sim \frac{4}{K}(\Delta k-\Delta \beta) \\
& D_{-1} \sim \frac{4}{K}(\Delta k+\Delta \beta+\delta) \\
& D_{-2} \frac{4}{K}(\Delta k+\Delta \beta-\delta)
\end{aligned}
$$

and the other D's are found to be of zeroth order. We now see that if equation (40) is expanded to the lowest order in the perturbation, it is reduced to the form

$$
\begin{gathered}
D_{0} D_{-1} D_{-2}-\left|f_{1}\right|^{2} D_{-1}-\left|g_{1}\right|^{2} D_{-2}=0 \\
n=\left|f_{1}\right| \quad n_{2}=\left|g_{1}\right|
\end{gathered}
$$

We can further expand (54) with the use of (53) to find the polynomial expression of (54), which can be written

$$
\begin{gathered}
(\tilde{\Delta} \beta)^{3}+\tilde{\Delta k}(\tilde{\Delta} \beta)^{2}-\tilde{\Delta} \beta\left[(\tilde{\Delta} k)^{2}+\tilde{\delta}^{2}-\frac{n_{+}^{2}}{64}\right. \\
-\tilde{\Delta} k\left[(\tilde{\Delta} k)^{2}-\tilde{\delta}^{2}-\frac{n_{+}^{2}}{64}\right]-\frac{\delta n_{-}^{2}}{64}=0 \\
\tilde{\Delta} \beta=\frac{\Delta \beta}{k} \quad \tilde{\Delta} k=\frac{\Delta k}{k} \quad \tilde{\delta}=\frac{\delta}{k}
\end{gathered}
$$

where 


$$
\begin{aligned}
& n_{+}^{2}=n_{1}^{2}+n_{2}^{2} \\
& n_{-}^{2}=n_{2}^{2}-n_{1}^{2}
\end{aligned}
$$

The real advantage of (55) over our earlier fifth degree polynimial is that (55) is of only third order in $\tilde{\Delta} \beta$. Being of third order, there exists an explicit formula for the polynomial's roots ${ }^{34}$, and therefore it is possible to follow each root separately through the coalesced region. The numerical technique is described in Appendix A.

Several plots are exhibited in figures 20-23. We see that results are essentially what we would have expected from physical considerations. As $\delta$ is gradually decreased from the initial value (the perturbation being kept constant), the bands come smoothly together, and in the limit of $\delta$ equal zero, it can be seen that the total bandgap width becomes

$$
\begin{aligned}
&\left.\Delta \mathrm{k}\right|_{\text {total }}=2 x_{\text {RMS }} \\
& x_{\text {RMS }} \frac{\sqrt{n_{1}^{2}+n_{2}^{2}}}{8}=\delta_{\text {critical }} k=\delta_{c}{ }^{k}
\end{aligned}
$$

indicating that the perturbations sum in a root mean square manner, an outcome to be expected from a system in which absolute phase is unimportant.

3) Periodic Perturbation Theory

To develop the periodic perturbation theory, we fix the basic period $k$ at unity, equate the basic period to $\Delta k$, the spacing between our perturbations, and to force the bands to come together we let $N_{k}$, the frequency of the lower perturbation, become 1arge. We can indicate 
83.

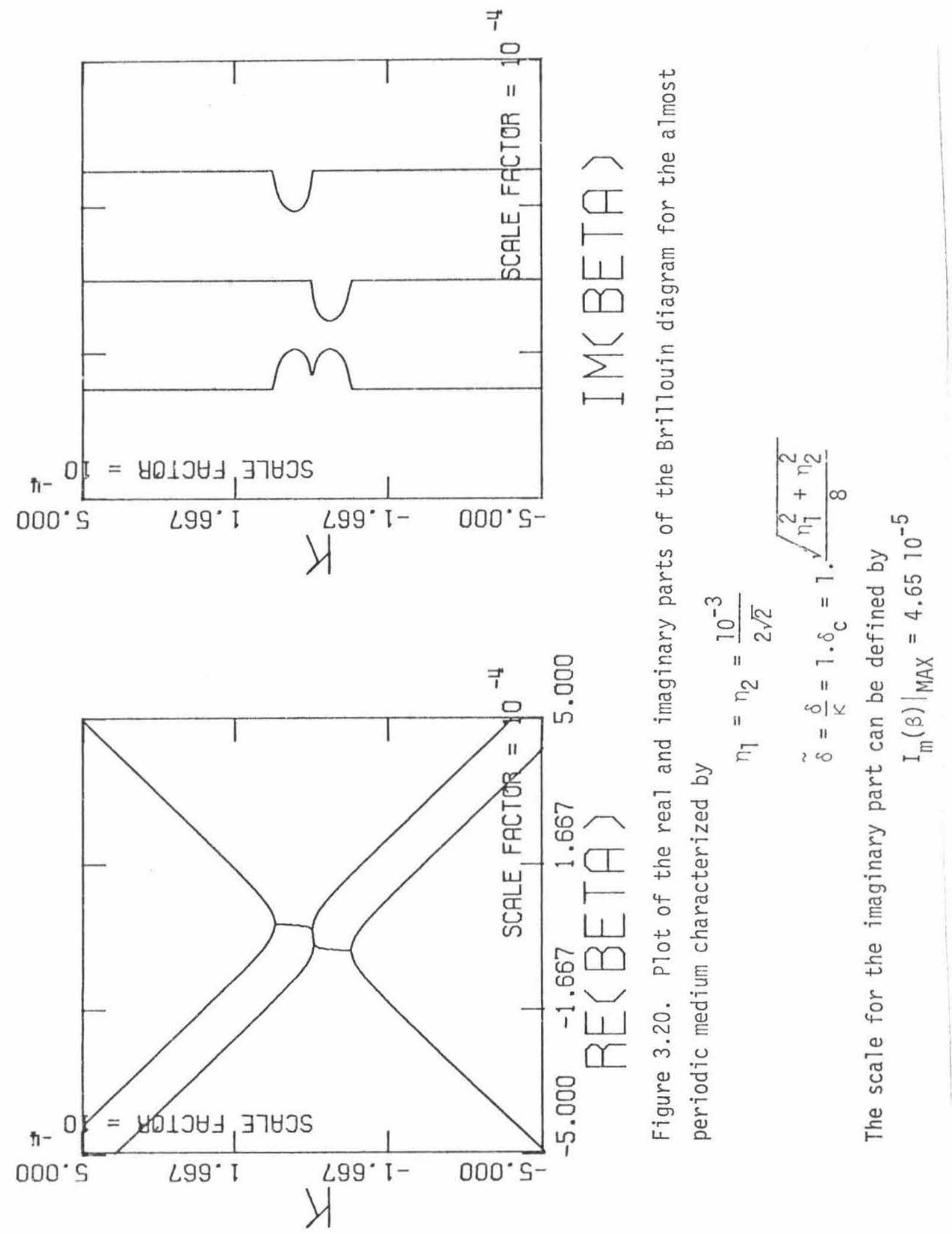


39.

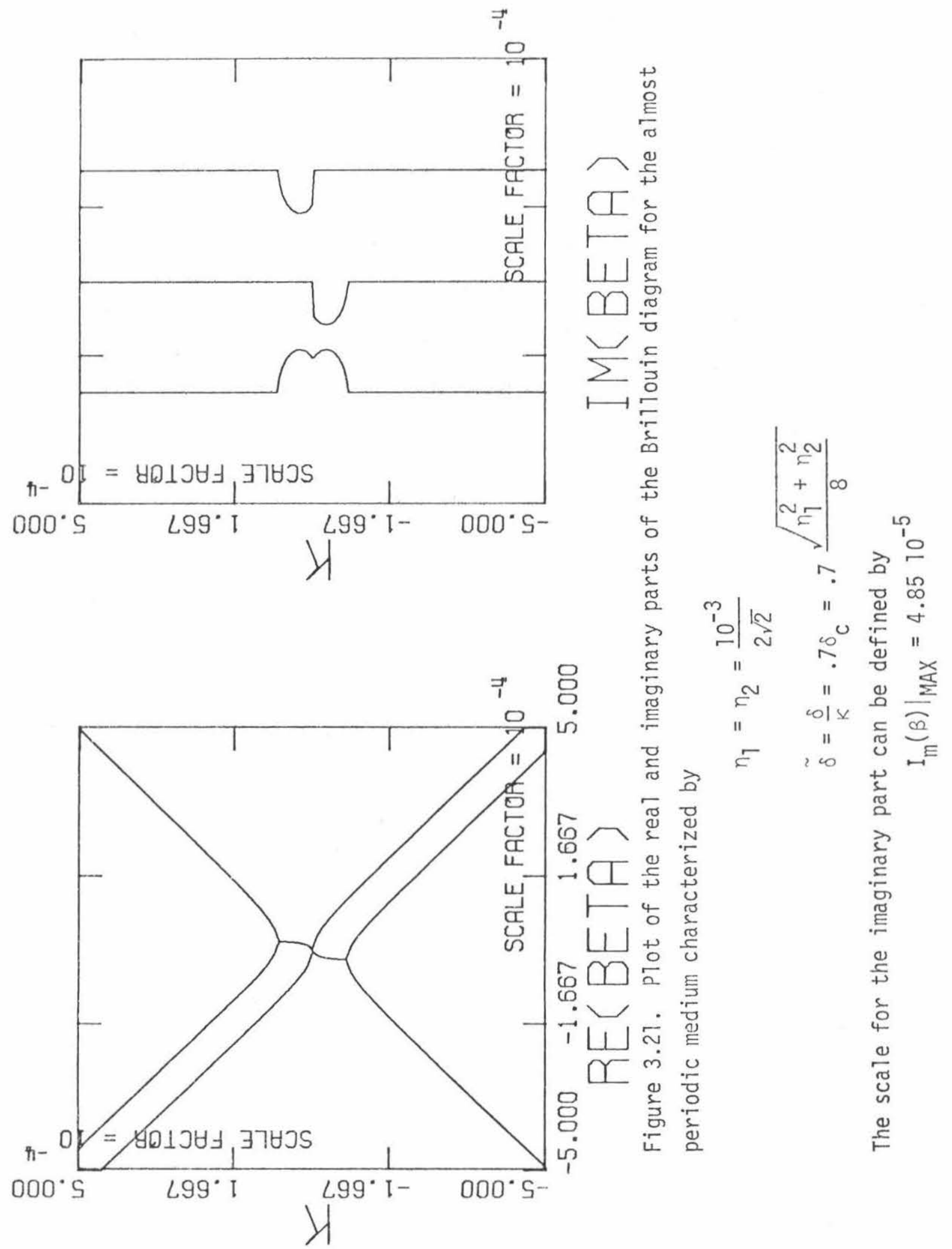


90.

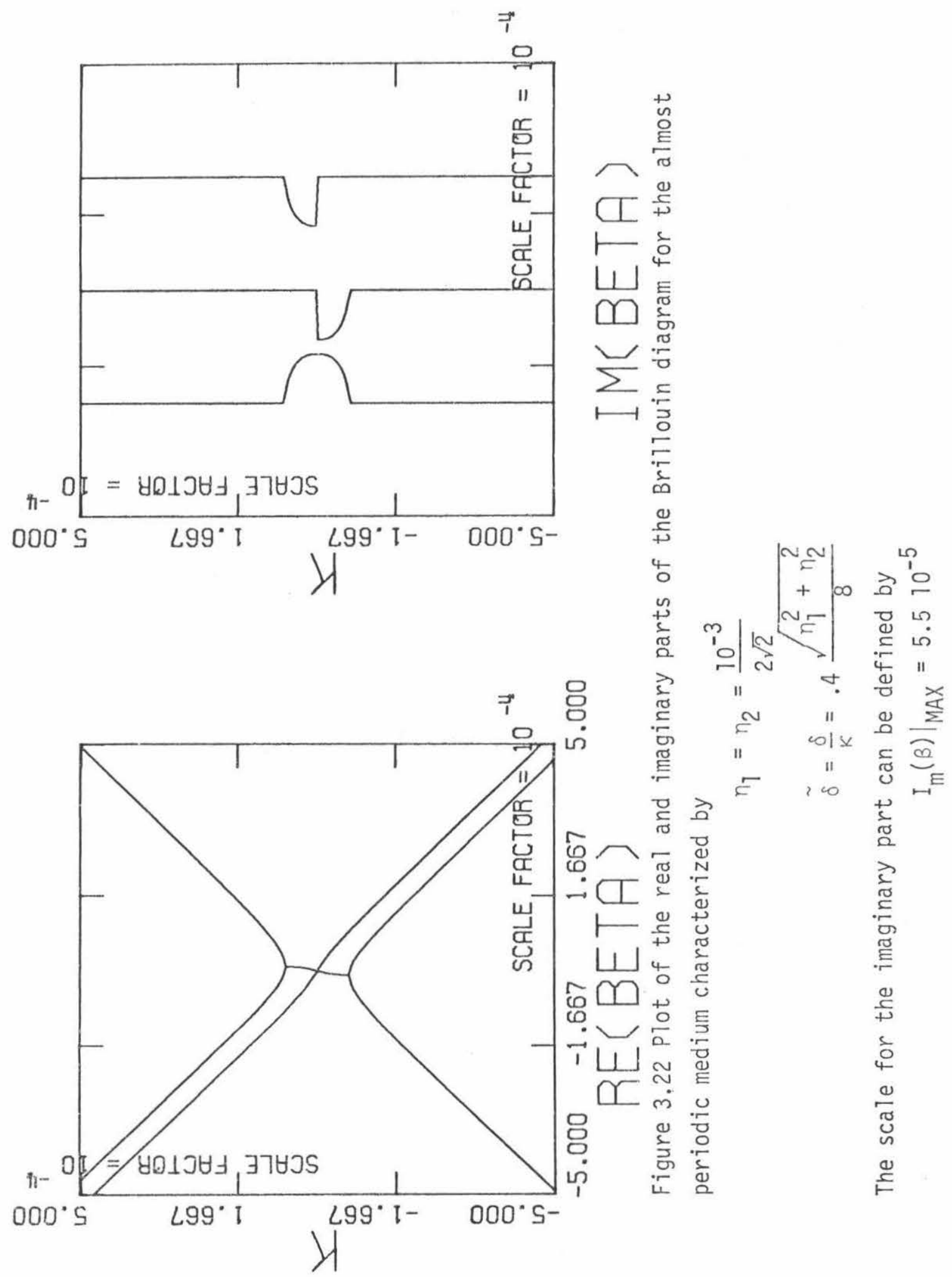


91.

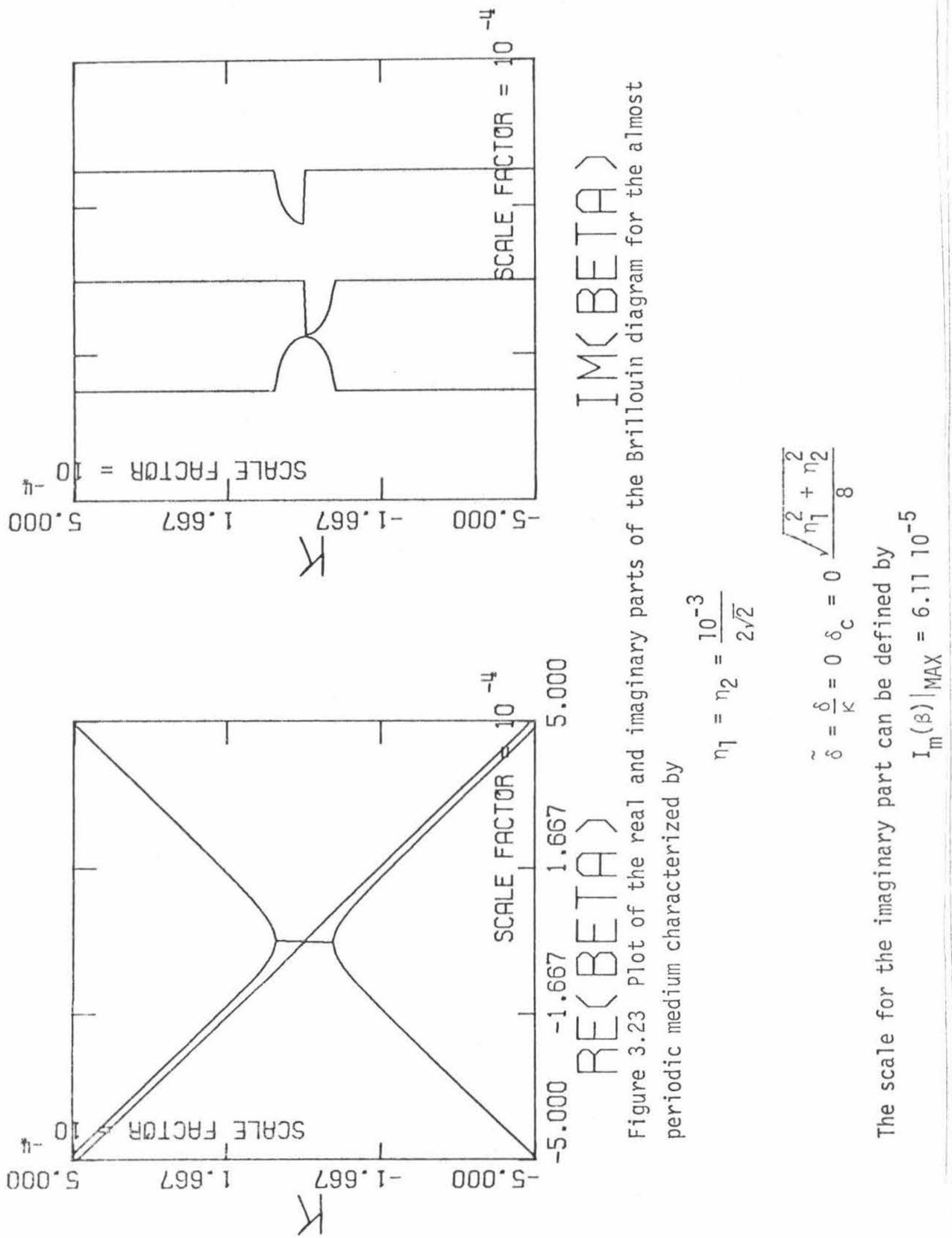


this coupling scheme on a schematic Brillouin diagram analogous to that of (32), as follows:

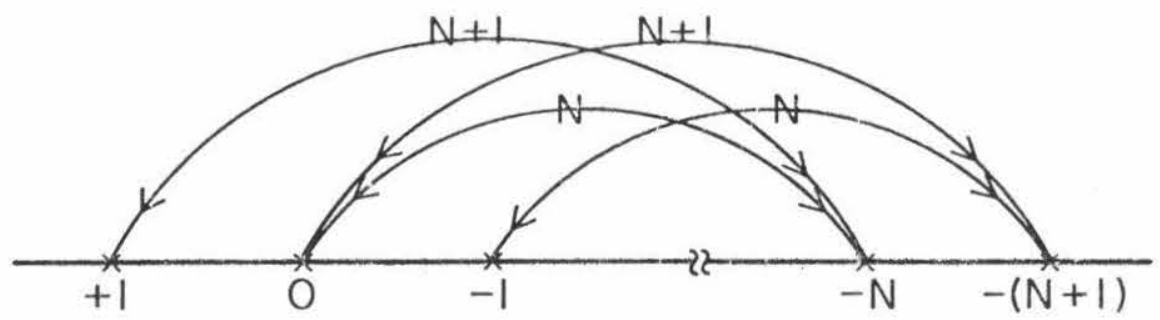

which represents the system of equations

$$
\begin{aligned}
& D_{1} a_{1}+\frac{n_{2}}{2} a_{-N}=0 \\
& D_{0} a_{0}+\frac{n_{1}}{2} a_{-N}+\frac{n_{2}}{2} a_{-(N+1)}=0 \\
& D_{-1} a_{-1}+\frac{n_{1}}{2} a_{-(N+1)}=0 \\
& D_{-N} a_{-N}+\frac{n_{1}}{2} a_{0}+\frac{n_{2}}{2} a_{1}=0 \\
& D_{-(N+1)^{a}-(N+1)}+\frac{n_{1}}{2} a_{-1}+\frac{n_{2}}{2} a_{0}=0
\end{aligned}
$$

Expanding the D's of equation (59), we find

$$
\begin{aligned}
D_{-(N+1)} & \simeq \frac{4}{\left(N+\frac{1}{2}\right) K}\left(\Delta K+\Delta \beta-\frac{K}{2}\right) \\
D_{-N} & \simeq \frac{4}{\left(N+\frac{1}{2}\right) K}\left(\Delta K+\Delta \beta+\frac{K}{2}\right)
\end{aligned}
$$




$$
\begin{aligned}
& D_{-1} \simeq \frac{4}{\left(N+\frac{1}{2}\right)_{K}}(\Delta k-\Delta \beta-K) \\
& D_{0} \simeq \frac{4}{\left(N+\frac{1}{2}\right)_{K}}(\Delta k-\Delta \beta) \\
& D_{1} \simeq \frac{4}{\left(N+\frac{1}{2}\right)_{K}}(\Delta k-\Delta \beta+K)
\end{aligned}
$$

The rather surprising thing here is that all five D's are of the same order. In fact, in general we can write

$$
D_{(0 \pm \ell)} \simeq \frac{4}{\left(N+\frac{1}{2}\right)_{K}}(\Delta K-\Delta \beta \pm \ell K)
$$

and

$$
\begin{aligned}
& D_{-(N \pm l)} \simeq \frac{4}{\left(N+\frac{1}{2}\right) k}\left(\Delta k+\Delta \beta \pm \frac{(2 \ell+7)}{2} K\right) \\
& \text { l integer }
\end{aligned}
$$

The result that the D's fall off only algebraically is indeed disturbing as it is not clear how to truncate the general system

$$
\underset{\sim}{\mathrm{a}} \underset{\sim}{a}=0
$$

However, if we arbitrarily cut off the system (63) with five harmonics as in (60), we find the system (assuming $N$ to be very large)

$$
\begin{aligned}
\Delta \beta a_{1}-(\Delta k+k) a_{1} & =\frac{n_{2} N_{k}}{8} a_{-N} \\
\Delta \beta a_{0}-\Delta k a_{0} & =\frac{n_{1} N_{k}}{8} a_{N}+\frac{n_{2} N_{k}}{8} a_{-(N+1)} \\
\Delta \beta a_{-1}-(\Delta k-k) a_{-1} & =\frac{n_{1} N_{k}}{8} a_{-(N+1)}
\end{aligned}
$$


94.

$$
\begin{gathered}
\Delta B a_{-N}+\left(\Delta k+\frac{k}{2}\right) a_{-N}=-\frac{n_{1} N k}{8} a_{0}-\frac{n_{2} N_{K}}{8} a_{1} \\
\Delta B a_{-(N+1)}+\left(\Delta k-\frac{k}{2}\right)_{-(N+1)}=-\frac{n_{1} N_{k}}{8} a_{1}-\frac{n_{2} N_{k}}{8} a_{0}
\end{gathered}
$$

which can be rewritten in the matrix form

$$
\underset{\approx}{(D} \omega \omega \underset{\approx}{I}) \underset{\sim}{a}=0
$$

where $\underset{\approx}{\mathrm{I}}$ is the identity matrix. As (65) is a matrix eigenvalue equation, we can obtain its solution numerically for any number of harmonics by simply augmenting the system (64). This we have done and we have plotted the results in figures 24-28, which should be compared with the results plotted in figures 18 and 19.

In figures 24 and 25 we use the values of the plots of figures 18 and 19 to obtain a direct comparison. Figures 18 and 24 are rather hard to compare, as the precise meaning of 24 is not completely clear. The figure seems to say there are three bandgaps, but they have not yet coalesced. One problem with figure 24 may be that the derivation of (64) requires restrictive assumptions about $\Delta \kappa$, perhaps limiting the system's usefulness to cases in which coalescence has already occurred. In comparing figures 19 and 25 we see that this may be the case, as 25 indeed resembles 19 closely in length, however, not so closely in the center frequency. This disagreement in center frequency is perhaps not so severe, as figure 19 includes the second order effects. For the parameters given, the coupling constant for second order couplings

$$
x_{2}=\frac{n^{2} N}{8} \sim .4
$$

which is indeed quite large and could lead to band-center offset. If $\mathrm{N}$ 
95.

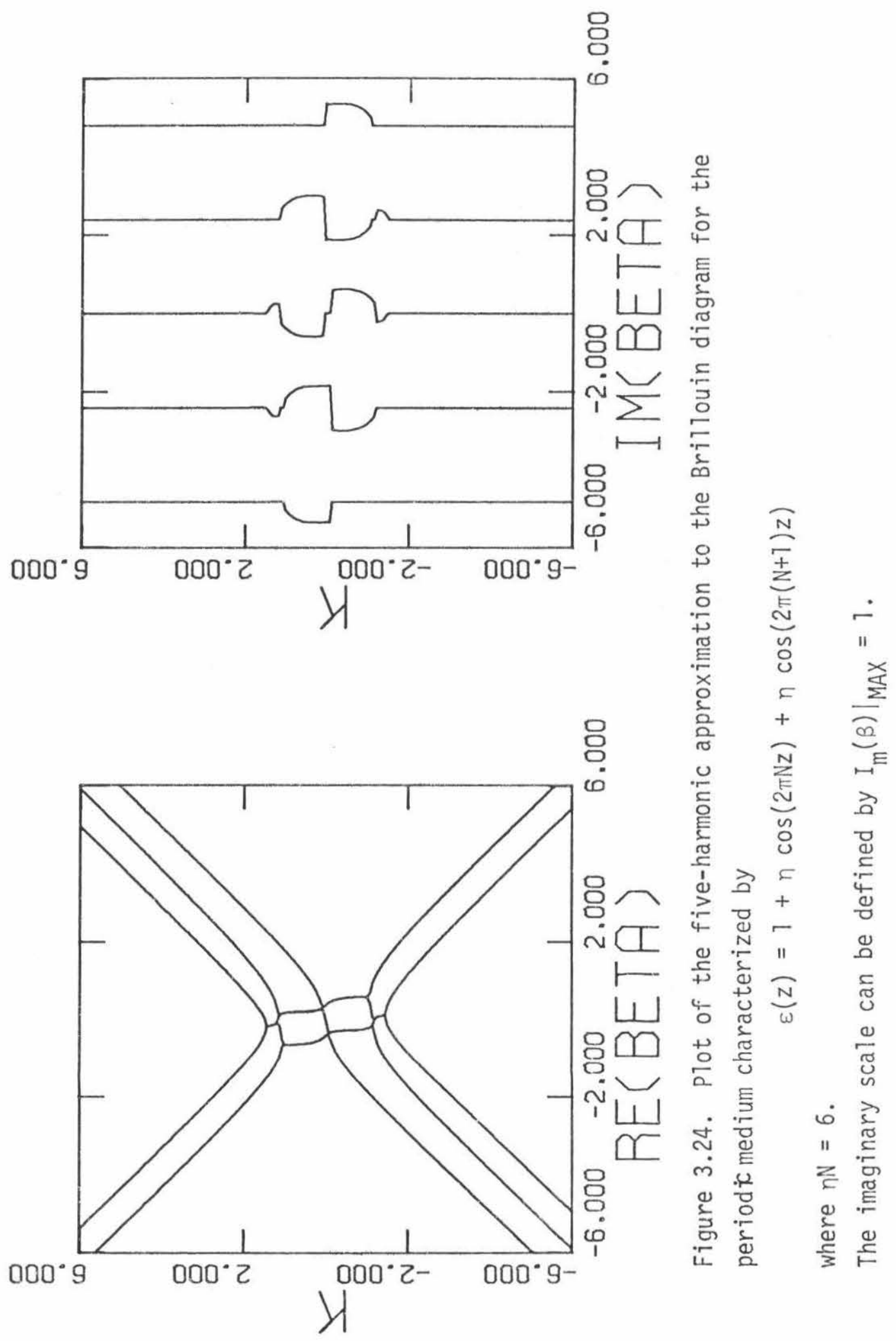


96.

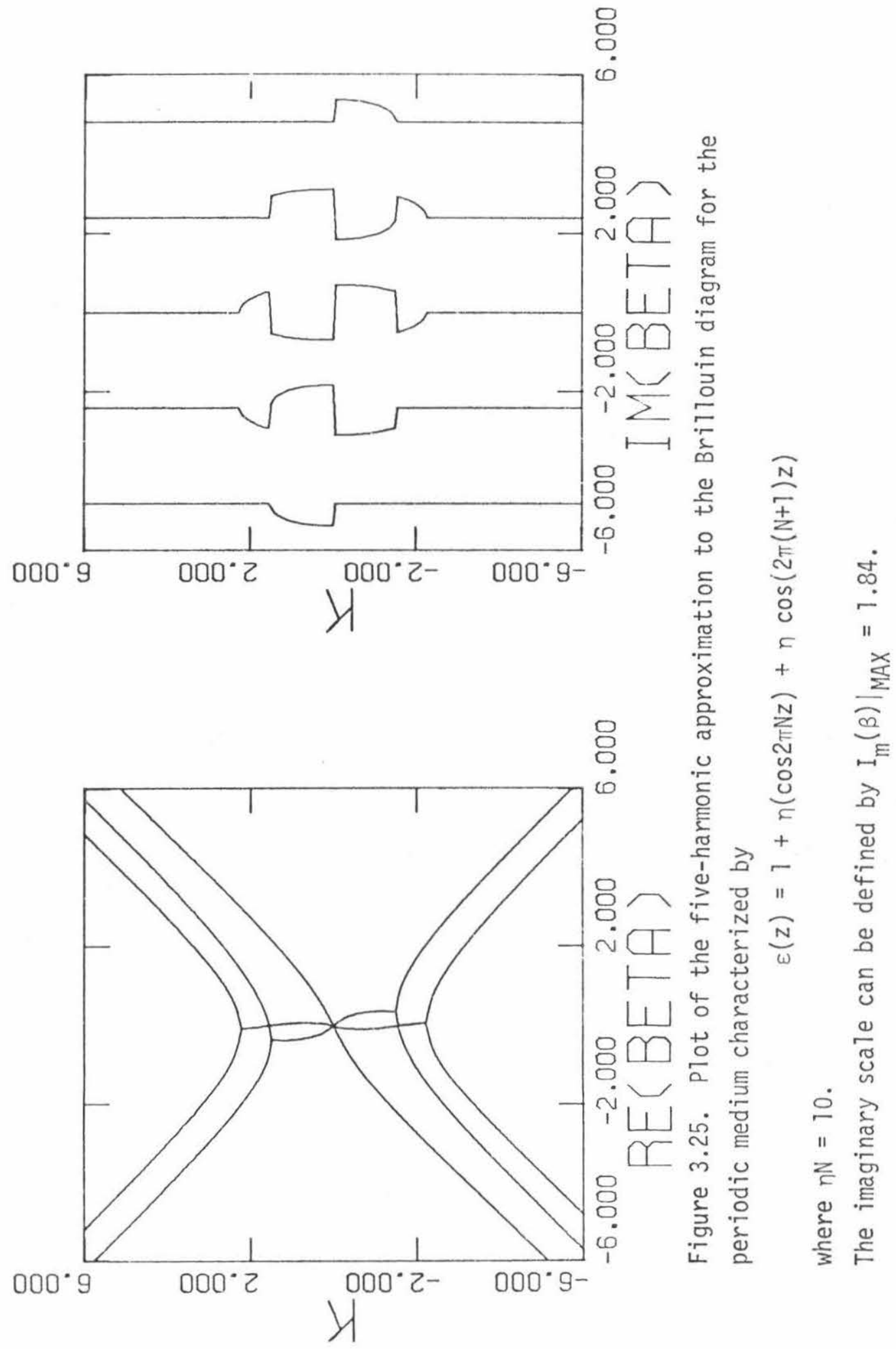




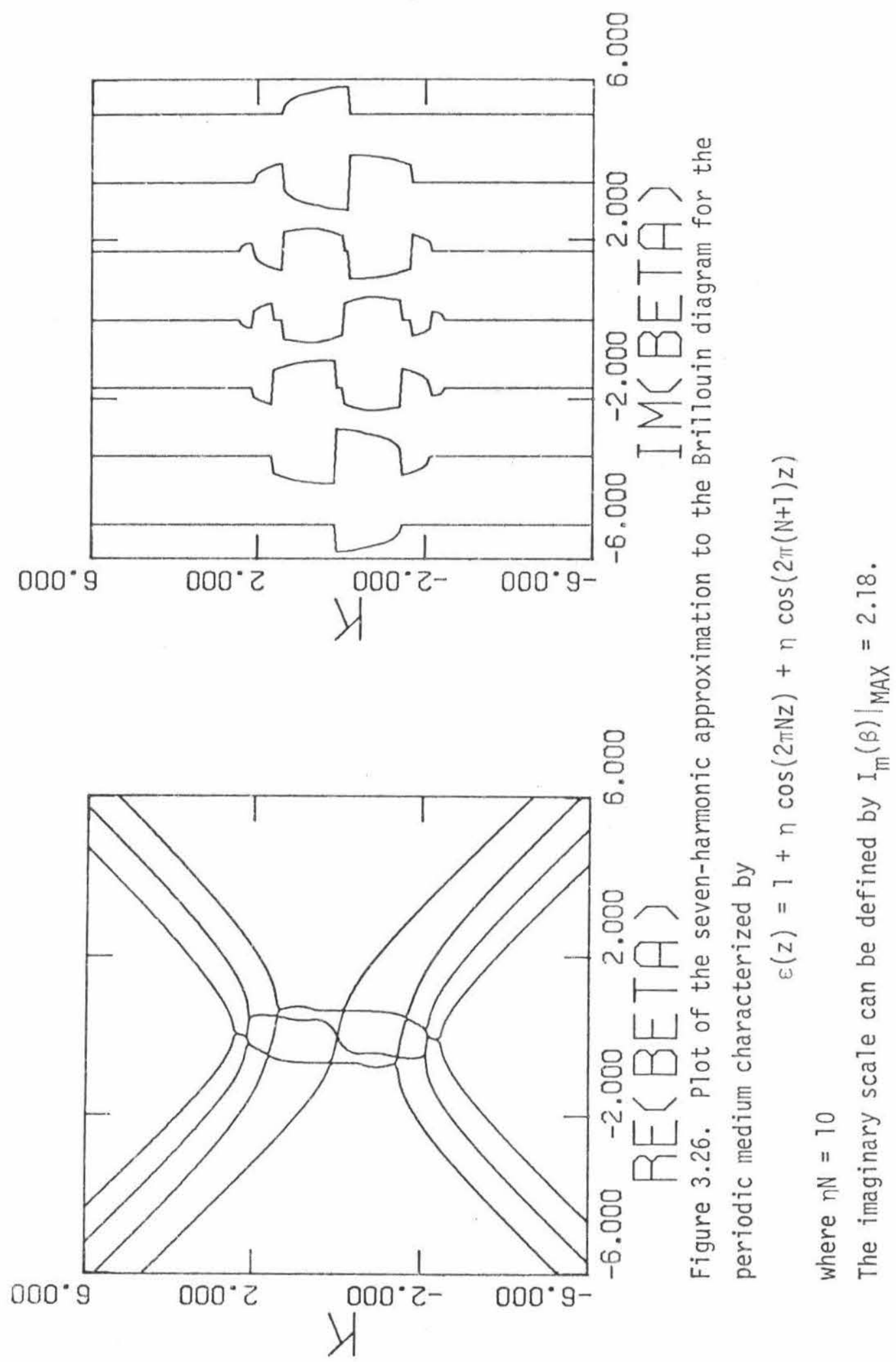


93.

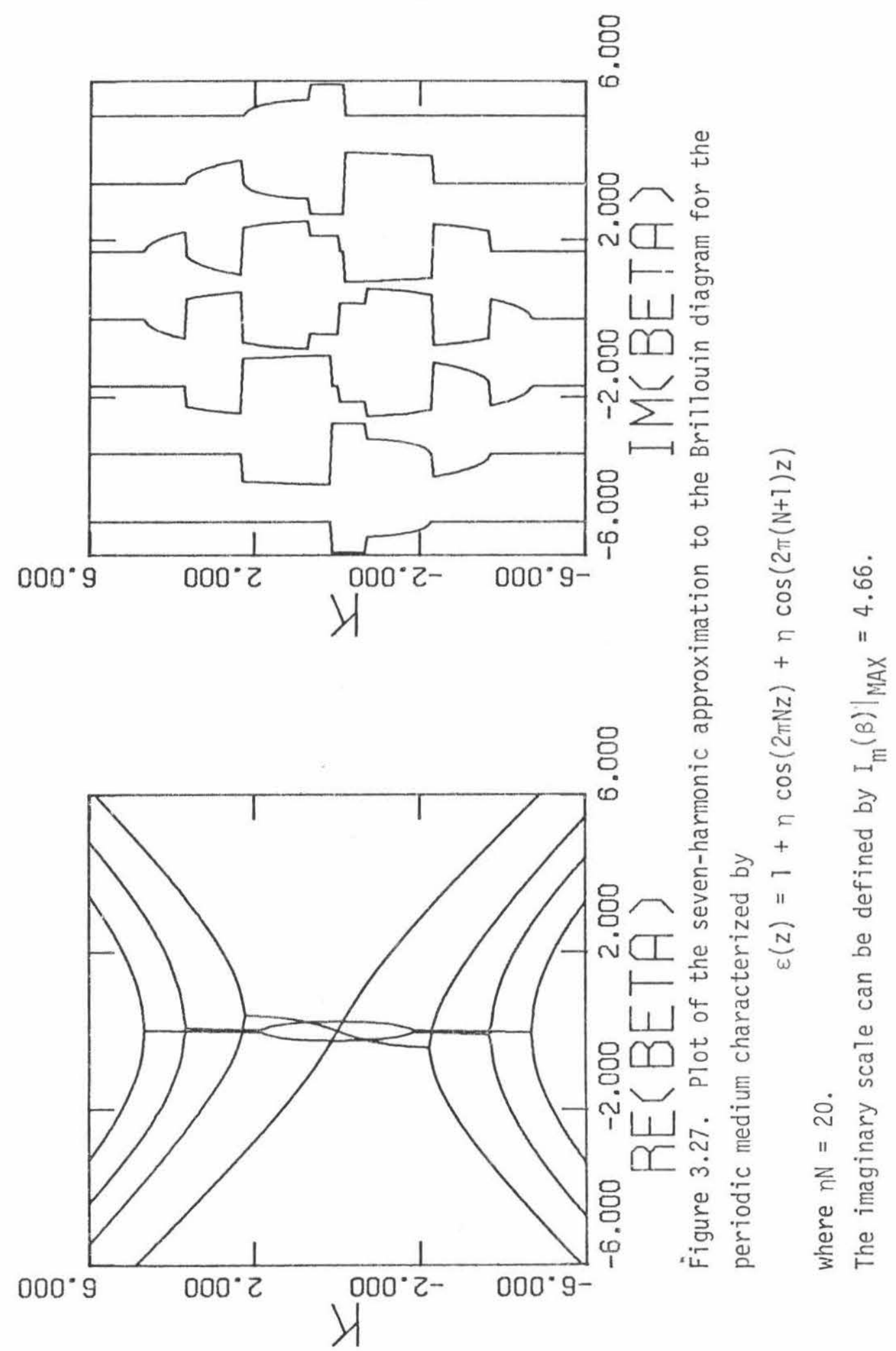


99.

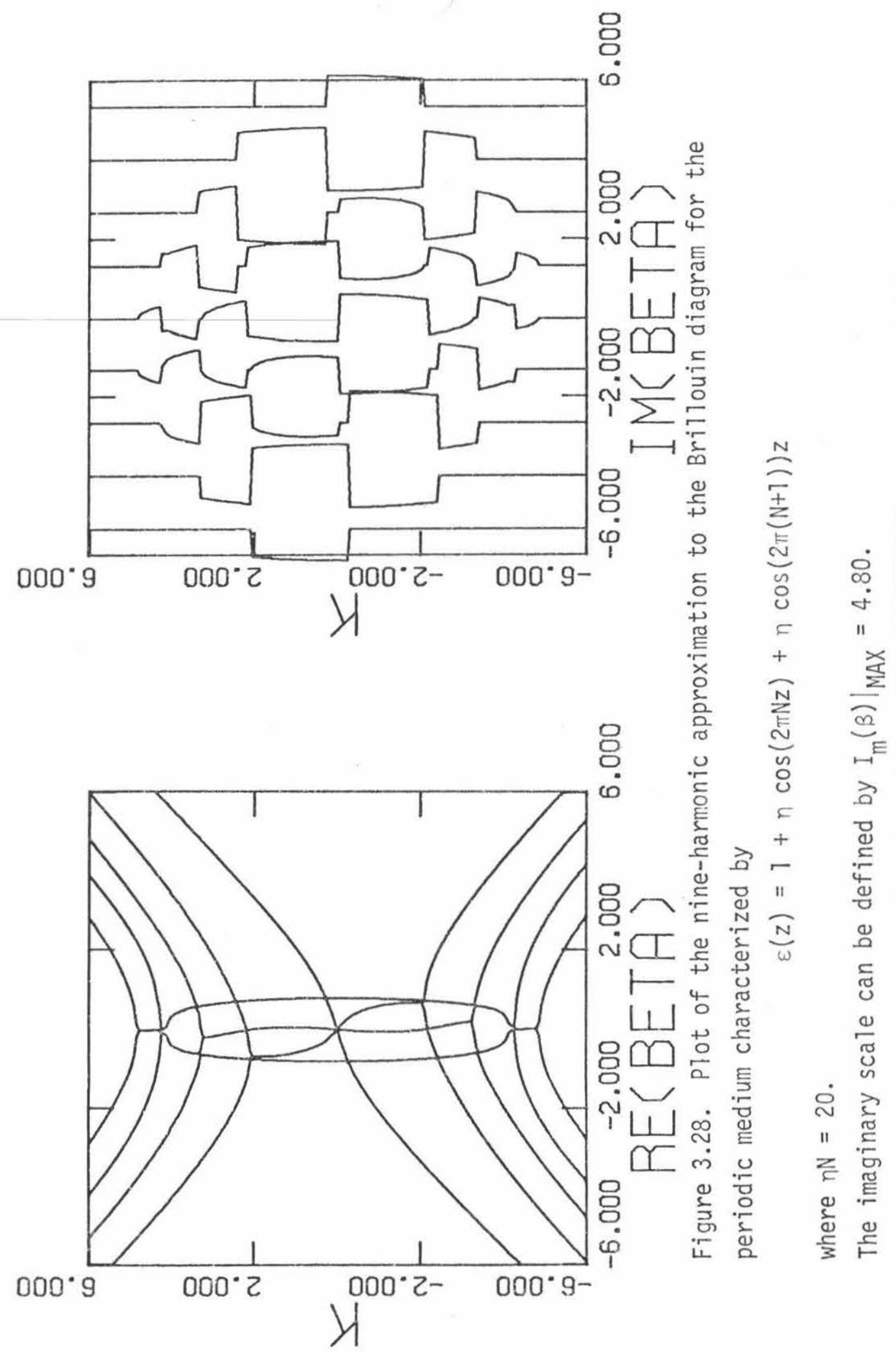


could be taken larger and $n$ smaller, the Floquet theory might well give results more comparable to figure 25 .

Figure 26 arises from the coupling scheme

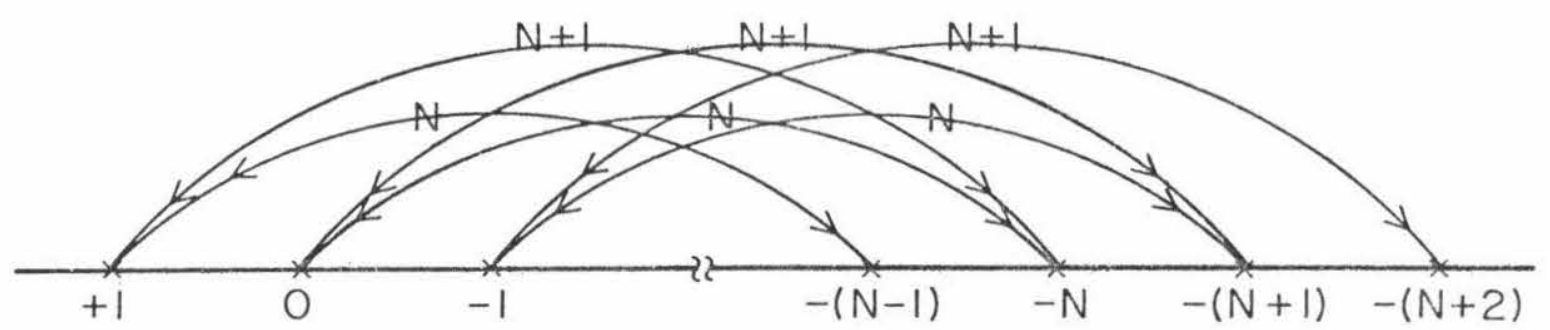

Figure 26 is plagued with the same disease as figure 24; that is to say, it is hard to interpret. It seems to be telling us that more harmonics are taking part in the interaction but that not all the bandgaps have yet coalesced. If we combine the results of figures 25 and 26 using this kind of interpretation, we decide that three bandgaps have coalesced,yet at least two more bandgaps exist. This interpretation seems to agree fairly well with the result of figure 19.

To check if our interpretation was correct, we raised the perturbation value to a higher level given by

$$
x=\frac{\mathrm{nN}}{8}=2.5
$$

and plotted the seven and nine-harmonic results in figures 27 and 28 . The nine-harmonic coupling scheme is given by 


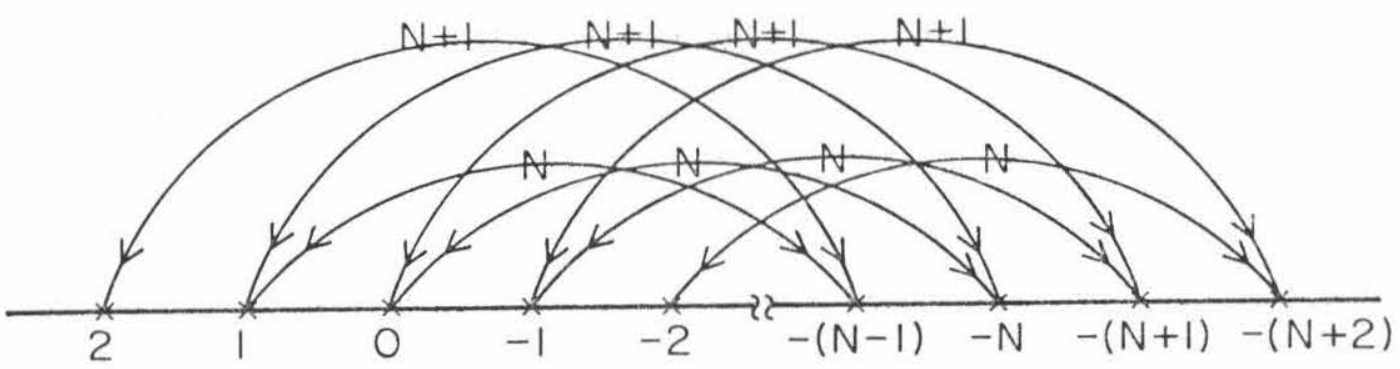

which is simply the symmetrical extension of (67) and (58). Figure 27 then exhibits the same behavior as figure 25, and figure 28 somewhat mimics figure 26. It seems that if our interpretation is correct, this perturbation would yield a five-bandgap coalescence with at least two other non-coalesced bandgaps appearing.

It appears we have unearthed a definite distinction between the periodic and almost periodic cases. In the last section of the next chapter we shall again address this problem of bandgap coalescence, although this time we will have both reflection and Brillouin diagram techniques at our disposal. As the reflection coefficient is the physically observable parameter, we feel it is best to defer the physical explanation of the above detected phenomena until after we have developed sufficient techniques with which to treat the reflection problem. 
1) L. Brillouin, Wave Propagation and Group Velocity (Academic Press, New York, N.Y., 1960).

2) J. C. S1ater, Rev. Mod. Phys. 20, 473-518 (1948).

3) L. Bril1ouin, J.A.P. 19, 1023-1041 (1948).

4) J. R. Pierce, Traveling Wave Tubes (D. Van Nostrand Co., Princeton, N.J., 1950).

5) V. L. Ginzburg, The Propagation of Electromagnetic Waves in Plasmas, (Pergamon Press, New York, N.Y., 1970).

6) Perhaps the best references for this are in the works of A. Yariv. See, for instance A. Yariv, Introduction to Optical Electronics (Rinehart and Winston, New York, N.Y., 1971), 2nd edition, Chapter 13; A. Yariv, Quantum Electronics (John Wiley and Sons, New York, N.Y., 1975), 2nd edition, Chapter 19.

7) C. Kittel, Introduction to Solid State Physics (John Wiley and Sons, New York, N.Y., 1976) 5th edition.

8) Kronig and Penney, Proc. Roy. Soc. Lond. A130, 499-513 (1930).

9) F. Bloch, Z. f. Phys. 52, 555-600 (1928).

10) L. Brillouin, J. de Physique I, 377-400 (1930).

11) L. Brillouin, J. de Physique $\underline{3}, 565-581$ (1932).

12) L. Brillouin, J. de Physique 4, 333-361 (1933).

13) J. C. S1ater, Phys. Rev. 45, 794-801 (1934).

14) L. Brillouin, Wave Propagation in Periodic Structures (Dover Pub., New York, N.Y., 1953). [Original edition, McGraw-Hi11 Book Co., 1946].

15) L. Brillouin, J. de Physique ㄱ, 401-410 (1936). 
16) See section 1 of reference (2).

17) Oliner and Hessel, I.R.E. Trans. on A.P., Special Supplement, Dec. 1959, pp. s201-208.

18) Cassedy and 01iner, Proc. IEEE 51, 1342-1359 (1963).

19) Many sources give good discussions of Bragg's 1aw. The one perhaps most appropriate to the discussion here is: D. L. Jaggard, Antenna Lab. Report No. 75, Caltech (1976).

20) Abramowitz and Stegun, Handbook of Mathematical Functions (Dover Pub., New York, N.Y., 1970), seventh printing. See equation 4.4.37.

21) J. R. Pierce, J.A.P. 25, 179-183 (1954).

22) J. R. Pierce, Proc. IRE 42, 1389-1396 (1954).

23) H. Kogelnik, Proc. Symp. Mod. Optics, Polytech. Inst., Brooklyn, N.Y. (March 1967).

24) H. Kogelnik, BSTJ 48, 2909-2947 (1969).

25) H. Kogelnik and C. V. Shank, J.A.P. 43, 2327-2335 (1972).

26) Sakuda and Yariv, Optics Comm. 8, 1-4 (1973).

27) H. Stoll and A. Yariv, Optics Comm. $\underline{8}$, 5-8 (1973).

28) A. Yariv, IEEE J. Quantum Electr. QE-9, 919-933 (1973).

29) A. Yariv and H-lW Yen, Optics Comm. 10, 120-122 (1974).

30) D. L. Jaggard and G. A. Evans, Antenna Lab. Tech. Report No. 73, Caltech (1975).

31) A. Yariv and A. Gover, App1. Phys. Lett. 26, 537-539 (1975).

32) D. L. Jaggard and C. Elachi, JOSA 66 , 674-682 (1976).

33) D. L. Jaggard and C. Elachi, JAP 48, 1461-1466 (1977).

34) D. L. Jaggard, App1. Phys. (Springer-Verlag) 13, 185-195 (1977).

35) See reference 20, equation 3.8.2, 


\section{CHAPTER IV}

The Reflection Coefficient

Although the discussion of the last chapter yielded information concerning the differences between periodic and almost periodic wave propagation, the information was always contained in a Brillouin diagram. The problem is now that we have no experimental technique to measure a Brillouin diagram. Although we feel that quantities on the Brillouin diagram relate to physically measurable parameters, such as the imaginary part of the bandgap telling us about the relative magnitude of the reflection coefficient, we have no firm tie-in. This chapter will attempt to mitigate this uncertainty by first considering the reflection coefficient in some depth and then developing a technique to find the reflection coefficient from the same equations with which we found the Brillouin diagram in the last chapter.

We begin the discussion by reminding the reader of some elementary considerations concerning reflection coefficients and perturbation expansions, essentially at the level of Morse and Feshbach. ${ }^{1}$ This discussion is designed to point out the problem of secularity in perturbation expansions, a problem which must later be overcome to obtain physically acceptable results for the reflection coefficient.

A) Standard Perturbation Theory for the Reflection Coefficient

1) Setting up the Exact Boundary Value Problem

Figure 1 defines the parameters and regions of the problem. We begin by writing the fields separately in each region: 


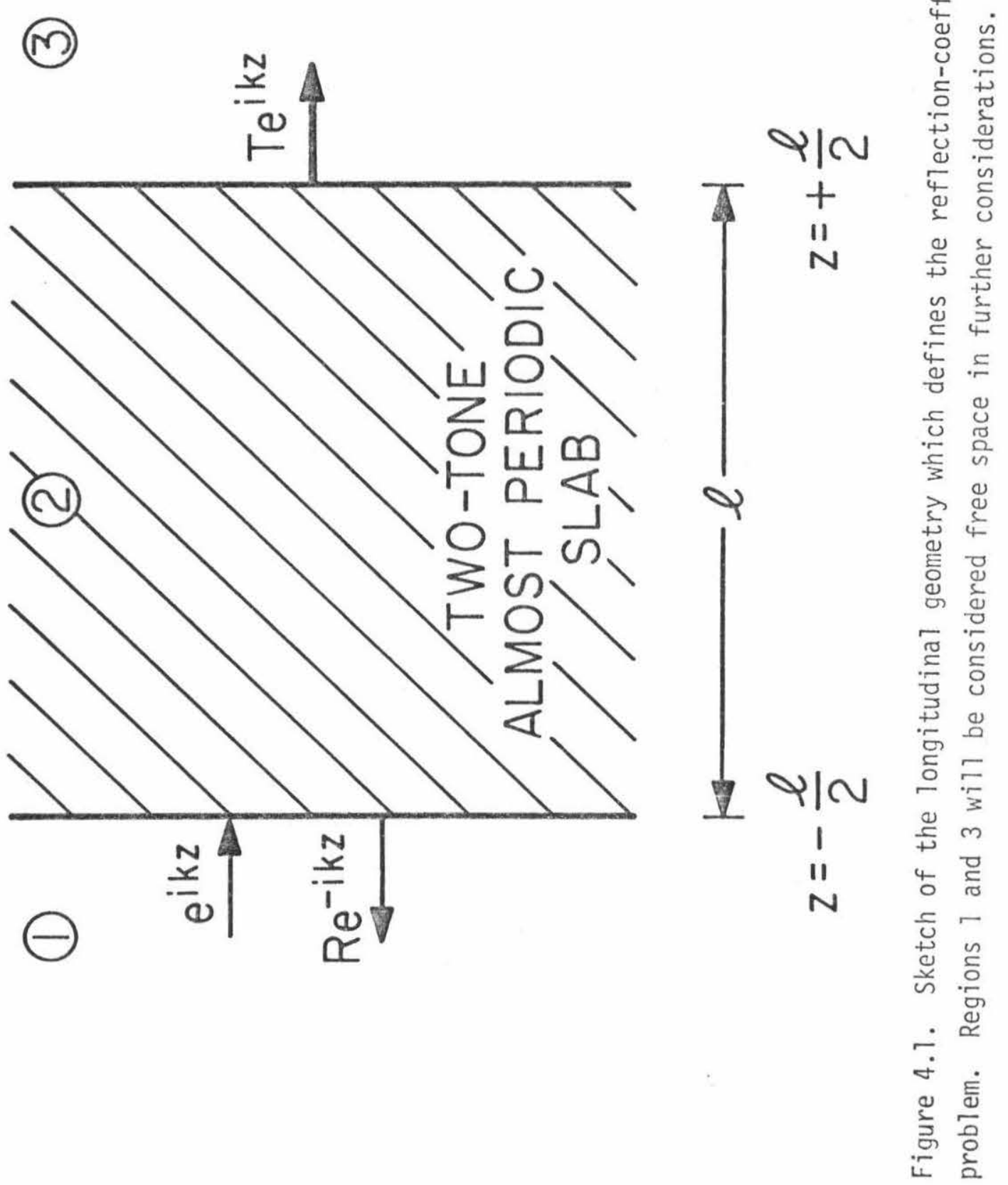


Region 1

$\psi=e^{i k z}+R e^{-i k z}$

Region 2

$\psi=\sum_{N, M} a_{N M}^{+} e^{i N \kappa_{1} z+i M K_{2} z} e^{i \beta z}+\sum_{N, M} a_{N M}^{-} e^{i N K_{1} z+i M K_{2} z} e^{-i \beta z}$

Region 3

$\psi=T e^{i k z}$

Note that we have assumed the almost periodic solution of Chapter II to be valid in region 2 .

Before equating the values and derivatives of $\psi$ across the boundaries, we must reduce the number of constants in region 2 through use of the dispersion relation. As $\psi$ satisfies a second-order differential equation, only two of the doubly infinite number of constants must actually be arbitrary. We proceed by solving the determinantal equation of Chapter III,

$$
\operatorname{det} \underset{\approx}{\mathrm{D}}=0
$$

for the doubly infinite set $\beta_{i j}^{2}$. As discussed in Chapter II, for each value of $\beta$, we can back-substitute into the equation

$$
\underset{\sim}{\mathrm{a}} \underset{\sim}{a}=0
$$

and obtain the $\underset{\sim}{a}$ 's as a one-parameter string, say in terms of $a_{00}$ as as

$$
\left.a_{N M}\right|_{\beta_{i j}}=\left.g_{N M}\left(\kappa_{7}, \kappa_{2}, \omega\right)\right|_{\beta_{i j}} a_{00}
$$


Say that for each of the positive (negative) roots of (4) we carry out the procedure indicated by (5) and (6), denoting the free parameter by $a_{00}^{+}\left(a_{00}^{-}\right)$. We then find the field in region 2 to be expressible as

$$
\begin{aligned}
& \psi=a_{00}^{+} \sum_{i, j} \sum_{N, M} g_{N M}\left(\kappa_{1}, \kappa_{2}, \omega\right) \mid+\beta_{i j} e^{i\left(N_{\kappa_{1}}+M k_{2}\right) z} e^{i \beta_{i j} z} \\
& +a_{00}^{-} \sum_{i, j N, M} \sum_{N M} g_{N M}\left(\kappa_{1}, k_{2}, \omega\right) \mid{ }_{-\beta_{i j}} e^{i\left(N \kappa_{1}+M \kappa_{2}\right) z} e^{-i \beta_{i j} z}
\end{aligned}
$$

We have reduced the boundary value problem to four equations (continuity of $\psi$ and its first derivative across two boundaries) in four unknowns $\left(a_{00}^{+}, a_{00}^{-}, R\right.$ and $\left.T\right)$. However, the sheer complexity of the system renders exact solution impossible. The need for some perturbation theory is evident.

2) Born-Neumann Series

Perhaps the most straightforward perturbation scheme to apply is that of Born ${ }^{2}$ and Neumann ${ }^{3}$. If we expand $\psi$ in our wave equation

$$
\frac{d^{2}}{d z^{2}} \psi+k^{2} \varepsilon_{r} \psi=-\frac{k^{2} \varepsilon_{r}}{2} \operatorname{AP}(z) \psi \quad-\frac{\ell}{2}<z<\frac{\ell}{2}
$$

where $\operatorname{AP}(z)$ is an almost periodic function in terms of the parameter $n$, and pick off the first order reflection coefficient $R$, we obtain the familiar form

$$
R\left(k \sqrt{\varepsilon_{r}}\right)=-\frac{i n k \sqrt{\varepsilon_{r}}}{4} f_{\beta^{\prime}}\left[\operatorname{AP}(z) \operatorname{rect}\left(\frac{z}{l}-\frac{1}{2}\right)\right]_{\beta^{\prime}}=-2 k \sqrt{\varepsilon_{r}}
$$

where 


$$
f_{\beta^{\prime}}\left[(f(z)]=\int_{-\infty}^{\infty} e^{-i \beta^{\prime} z} f(z) d z\right.
$$

We have found this Fourier transform property to be a more general property of scattering than before stated in the literature, and in Appendix B we show it to be true for three dimensional scattering in very general constitutive media. However, in spite of the generality, simplicity and invertibility of this result, we will presently show it is not of much practical interest in our case.

3) Secularity of Born-Neumann Series at Bragg Resonance

Consider the simplest of almost periodic functions (periodic functions being a subclass)

$$
A P(z)=\cos k z
$$

A simple computation with (11) yields the result, noted elsewhere ${ }^{j}$, that

$$
R\left(k \sqrt{\varepsilon_{r}}\right)=\frac{i k \sqrt{\varepsilon_{r}}}{4}\left[\frac{\sin \left(k \sqrt{\varepsilon_{r}}+\frac{k}{2}\right) l}{\left.k \sqrt{\varepsilon_{r}}+\frac{k}{2}\right) l}+\frac{\sin \left(k \sqrt{\varepsilon_{r}}-\frac{5}{2}\right) l}{\left(k \sqrt{\varepsilon_{r}}-\frac{k}{2}\right) l}\right]
$$

If we investigate the maximum of this equation, which occurs for

$$
k \sqrt{\varepsilon_{r}}=\frac{\kappa}{2}
$$

the longitudinal Bragg condition, we find

$$
R\left(\frac{k}{2}\right)=\frac{i n k \sqrt{\varepsilon_{r}}}{4}=\frac{i n k \ell}{8}
$$

a most disconcerting result. The maximum value increases linearly in both the frequency and the length of the medium, not just in the perturbation. It is as if we expanded in the quantity nke instead of $n$ an effect 
commonly termed secularity ${ }^{6}$. It appears we are stuck with a theory that breaks down as soon as we reach the regime of interest where there is strong wave-medium coupling.

It is easy to check that the problem does not improve in the almost periodic case. Nor does it really improve by use of the various renormalization techniques ${ }^{7}$ designed to remove secular terms, as the increase in complexity leads to no certain increase in accuracy ${ }^{8}$. Further, the method is not designed to show any difference between periodic and almost periodic media. Clearly a new theory is required.

\section{B) The Electromagnetic Riccati Equation}

The first and perhaps most basic result of the technique of invariant imbedding is that of the Riccati equation for the electromagnetic reflection coefficient. This section is intended not only to introduce the reader to the basic precepts of this technique, but also to develop and discuss this exact reflection coefficient equation whose numerical solution we shall later employ. We begin with a brief historical perspective on the invariant imbedding technique.

1) Invariant Imbedding--Historical Perspective

The invariant imbedding technique was first applied by Sir George Gabriel Stokes in an 1862 work $^{9}$ considering the propagation of light through a striated medium. Some fifty years later, Lord Rayleigh 10,11 somewhat extended the technique in investigations of a similar problem. H. W. Schmidt ${ }^{12}$ was the first to continuously vary the length of a medium rather than simply add a discrete slab as had Stokes and Rayleigh. In spite of Schmidt's 1907 study of $\beta$-decay, however, the first 
generaliy recognized work of invariant imbedding is that of $V$. A. Ambartsumian in $1945^{13}$. In this work as in his 1958 treatise $^{14}$, Ambartsumian employed the invariant imbedding technique to develop a theory of radiative transfer. Although Ambartsumian's work stimulated certain workers in the West ${ }^{15}$, it was Chandresekhar's unifying treatment in his 1960 book $^{16}$ on radiative transfer that brought the concept to a research forefront. Coupling Chandresekhar's book with the earlier developed functional equation techniques of Redheffer ${ }^{17}$, various workers in mathematics and physics then solved a plethora of problems in numerous areas as evidenced by the reference 1 ists in recent books ${ }^{18}$ on the topic. Of the numerous references on the topic we have found that of Bellman and Wing ${ }^{19}$ to be the most useful and in most of what follows we adhere somewhat to their notation and conventions.

2) Stokes Recursion Relation and the Conservation of Energy It was prior to the publication of Maxwell's electromagnetic theory ${ }^{20}$ that Stokes solved the problem of wave propagation in a layered medium. He considered a geometry similar to that of figure and was armed essentially only with the Fresnel relations (refer to figure (2b) for directional definition) 


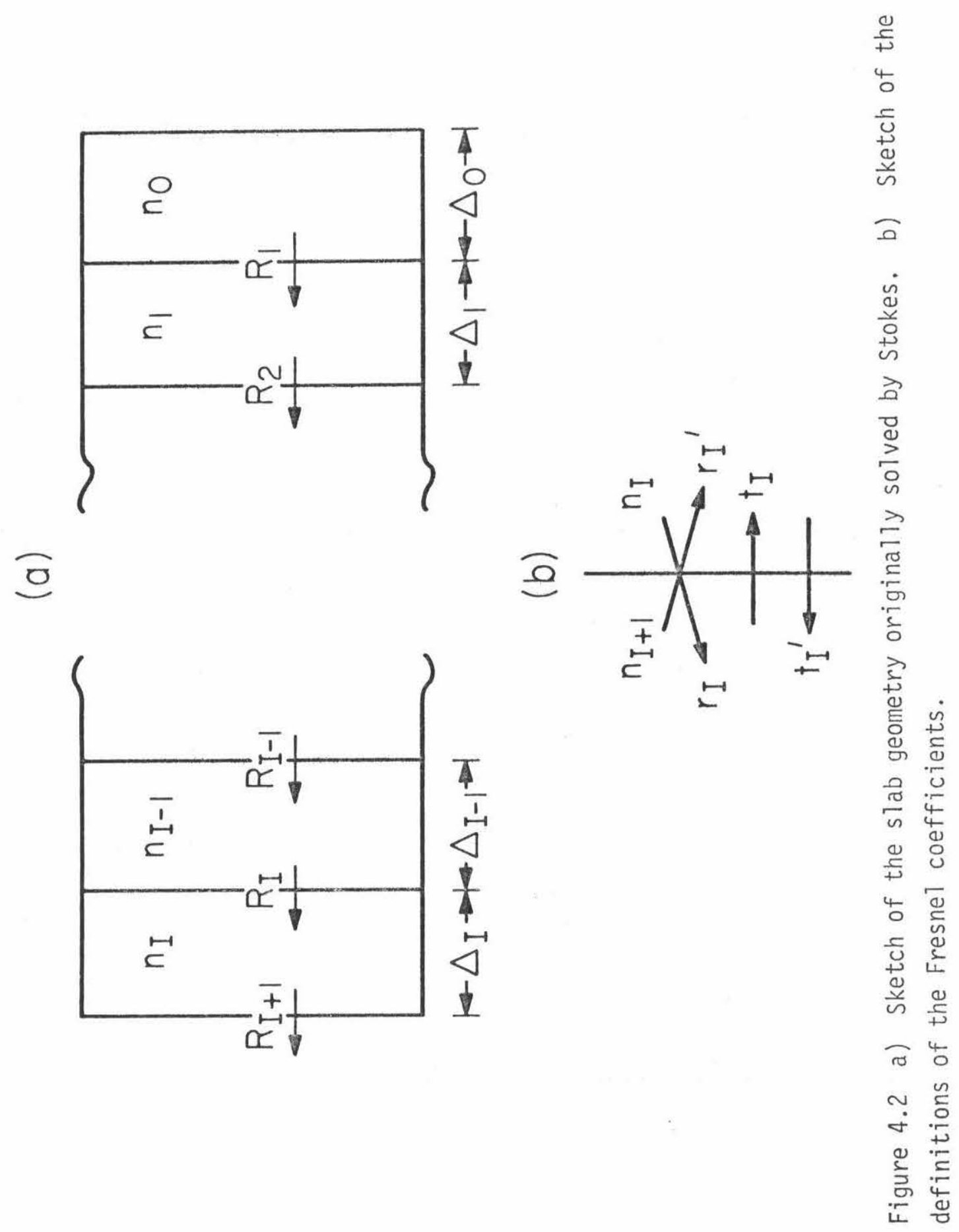




$$
\begin{aligned}
& r_{I}=\frac{n_{I+1}-n_{I}}{n_{I+1}+n_{I}} \\
& t_{I}=\frac{2 n_{I+1}}{n_{I+1}+n_{I}}=1+r_{I} \\
& r_{I}^{\prime}=\frac{n_{I}-n_{I+1}}{n_{I}+n_{L+1}}=-r_{I} \\
& t_{I}^{\prime}=\frac{2 n_{I}}{n_{I}+n_{I+1}}
\end{aligned}
$$

The technique employed was similar, but not equivalent, to the multiple-bounce argument ${ }^{21}$ used for the solution of the single slab problem. The essential difference lay in the use of the invariant imbedding approach. Stokes assumed he knew the solution to the $i$ layer problem, added an $(i+1)^{\text {th }}$ layer, summed the added contributions to the reflection arising from the $(i+1)^{\text {th }}$ layer as in the multiplebounce argument, by assuming that each time a wave traversed the $(i+1)^{\text {th }}$ layer and impinged on the $i^{\text {th }}$ layer, it acted as a source for the reflection coefficient to the $i$ layer problem. The technique is basic to invariant imbedding and is indicated schematically in figure (3). As the details are carried out elsewhere ${ }^{19}$, without further ado we write the result that

$$
R_{I+1}=\frac{r_{I}+e^{2 i k}{ }^{\Delta} I_{R}}{1+r_{I} e^{2 i k_{I} \Delta_{I}} R_{I}}
$$

where 
113.

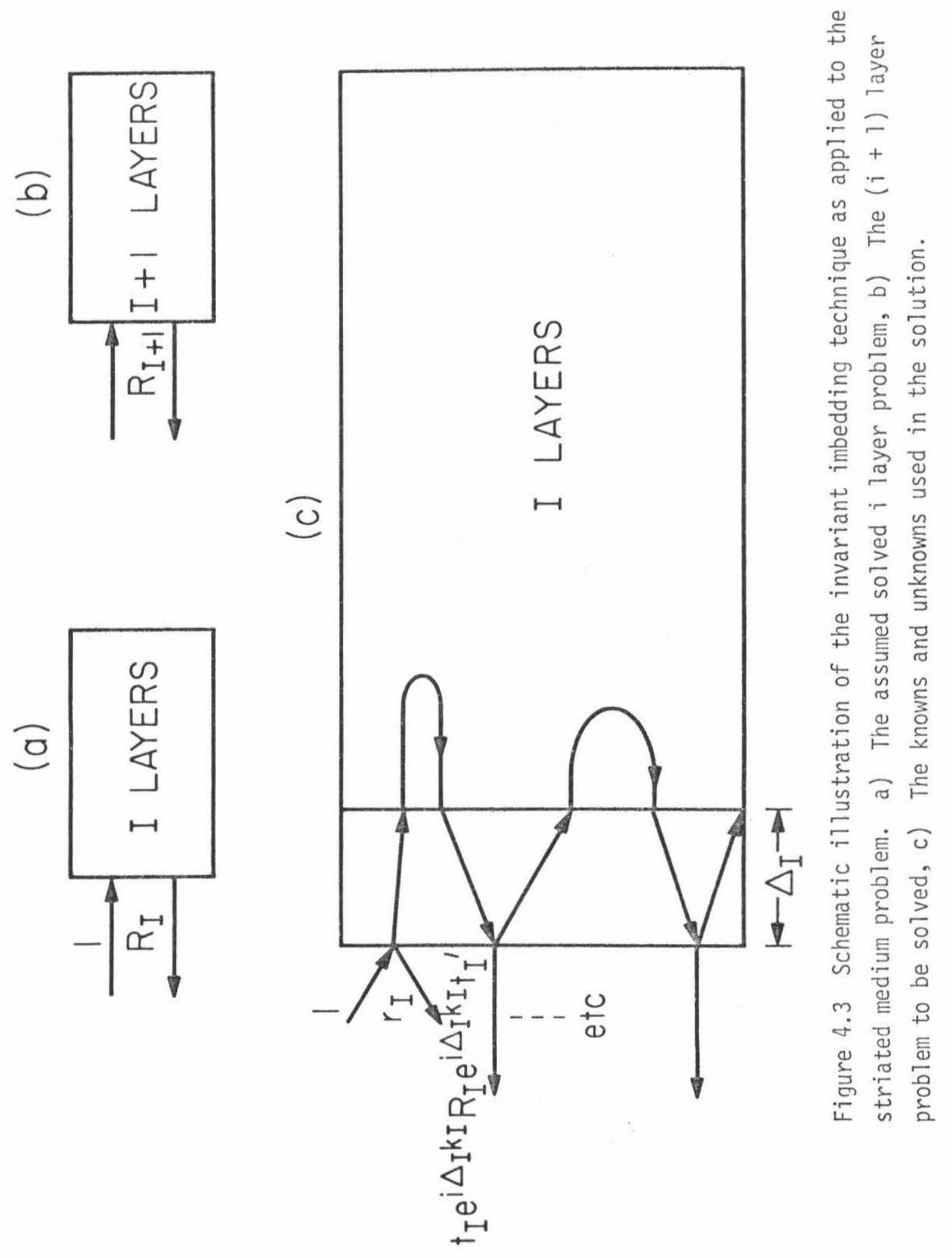




$$
k_{I}=\frac{2 \pi n_{I}}{\lambda_{0}}, \lambda_{0} \text { is the free space wavelength. }
$$

The recursion relation (16) is sufficient to solye the problem given some initial condition such as

$$
R_{0}=0
$$

Further, as Fresnel's formulas are rigorously derivable from Maxwell's equations, equation (16) represents an exact solution to the propagation problem.

of primary interest in this is the property of non-secularity and/or energy conservation. As (16) is an exact solution to a propagation problem, somehow it should include energy conservation. To indicate that this may be the case, we consider the following argument. If at some interface I the reflection coefficient has a magnitude of unity and some phase angle $\phi$, we find that at the next interface

$$
R_{I+1}=e^{2 i k_{I} \Delta_{I}} \frac{e^{i \phi}+r_{I} e^{-2 i k_{I} \Delta_{I}}}{1+r_{I} e^{2 i k_{I} \Delta_{I}} e^{i \phi}}=e^{i \phi^{\prime}}
$$

where $\phi^{\prime}$ is some new phase angle. The argument indicates that the reflection coefficient can never exceed the value one, and indicates that (16) certainly has the attributes of energy conservation, as it shoutd. 


\section{3) The Electromagnetic Riccati Equation}

The most straightforward generalization of Stokes' recursion relation is to allow $\Delta$ to go zero and thereby obtain a differential equation valid for media in which the refractive index varies continuously. Such a generalization is easily affected ${ }^{19}$ and the result found to be

$$
\frac{d R}{d z}=\frac{1}{2}\left(1-R^{2}\right) \frac{d k(z)}{d z} \frac{1}{k(z)}-2 i k(z) R
$$

where $z$ is the longitudinal coordinate and

$$
k(z)=\frac{2 \pi n(z)}{\lambda_{0}}
$$

Equation (19) has the well-known form of a generalized Ricatti equation $^{22}$.

The energy-conservation argument culminating in (18) can also be applied here and will result in a real equation for the variation of the phase angle with longitudinal coordinate, again indicating that (19), when subject to a reasonable initial condition, will not allow the reflection coefficient to exceed one. The solution to the inhomogeneous slab problem is completed.

The problem is, however, that (19) is a non-constant coefficient, non-linear differential equation which in general is not solvable. In Appendix $C$ we derive some interesting properties of the equation in genera1. In this appendix we also show that attempted perturbation expansions of (19) for the periodic and almost periodic 
cases contain first-order secularities and are therefore of little use. Essentially, the only way to solve (19) or (16) is numerically. This we have also done and found the solution to (16) to be considerably faster for comparable accuracy.

Armed with a numerical solution, one might think the problem is completed: only specific cases need be run off to compare the two solutions whose difference is our primary concern. This is not true for two reasons. First, the computer program becomes excessively expensive to run when more than a few periods of the dielectric constant are included in the medium, yet to find any difference between periodic and almost periodic media at all it is necessary to include many periods, lest the phase between the "bumps" be relatively unimportant. Second, small numerical round-off errors will cause an almost periodic equation to appear essentially periodic, at least for small numbers of periods, which is to say that there is no provision in the Riccati equation or computer to differentiate the cases. Although the Riccati equation is a powerful tool in checking perturbation solutions in its own regime of validity, it is not the definitive solution to the problem. However, as we shall see in the next section, in the derivation of the electromagnetic Riccati equation, i.e., the technique of invariant imbedding, lie the seeds of a method to both circumvent the problem of secularity and further illustrate the difference between periodic and almost periodic structures. 
C) A Generalized Look at Invariant Imbedding

In this section we will generalize an argument due to Bellman and Wing ${ }^{19}$ in two ways. First, we shall make the derivation applicable to arbitrary numbers of forward and backward waves, allowing the resulting system of equations to fit the form of the coupled equations we derived in the last section of chapter III. Secondly, we will apply generalized energy-conservation criteria to the characteristic parameters of the problem to deduce under what conditions we can be assured of a unitary (energy conserving) solution.

1) First-Order Wave Equations from Particle Counting Consider the diagram of figure (4). We wish to obtain the "particle flux" (field flow) through the medium in terms of forward and backward moving streams. We will assume that there are M distinguishable states (modes) moving to the right, generically denoted the forward wave, and $\mathrm{N}$ distinguishable states (modes) moving to the left, generically denoted the backward wave. The physical situation could be that of particles with spin moving down an accelerator, or that of energy being propagated through a ribbed multimode fiber-optic waveguide. The anisotropy between forward and backward waves could be due to a magnetic field in the accelerator, or an anisotropic ribbing structure in the lightguide. Whatever the situation, we wish to consider the change in the forward and backward waves as they traverse a tiny distance $\Delta$ in the media, a quantity which is later allowed to go to zero. First, let us consider the contributions to the forward 
113.

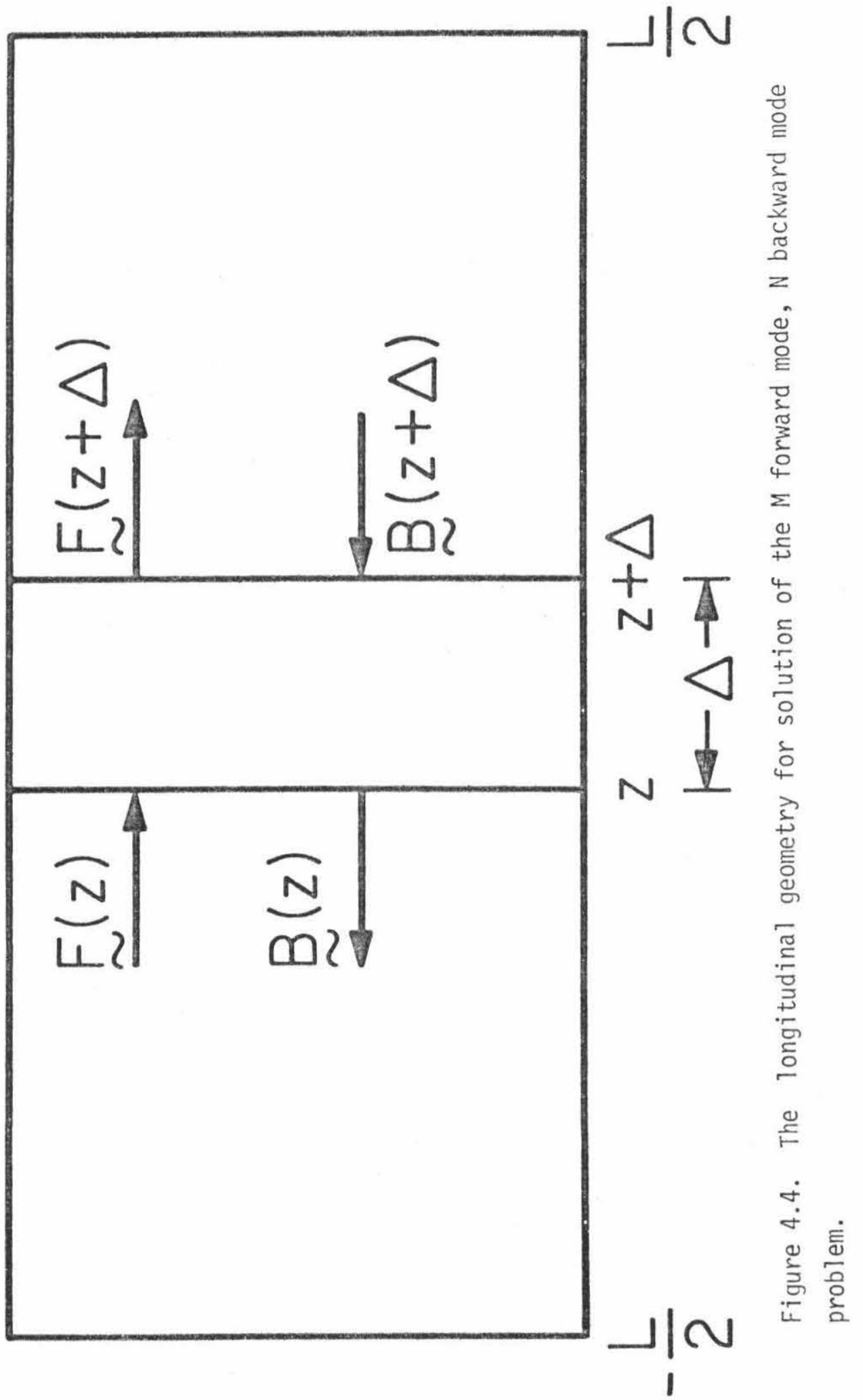


wave which leaves our test region at $z+\Delta$. Certainly, it receives a contribution from the impinging forward wave at $z$, which we shall write in the form

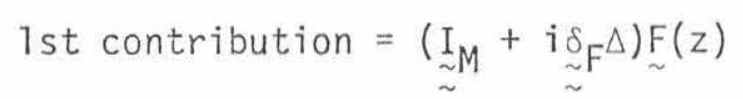

where ${\underset{\sim}{M}}_{M}$ is the MXM identity matrix and ${ }_{\sim}^{\delta} F$ part denotes phase shift per unit length $\tilde{\sim}$ and whose imaginary part denotes medium gain or loss per unit length. Note that in equation (21) we have only considered quantities of first order in $\Delta$ in anticipation of a later limit. We have still to include the contribution from the backward wave impinging on $z+\Delta$ from the left which gets backscattered in traversing the infinite-simal slab. This contribution is denoted by

$$
\text { 2nd contribution }=i \underset{\sim}{\mathrm{X}} \mathrm{FB}_{\mathrm{B}} \Delta \underset{\sim}{\mathrm{B}}(\mathrm{z}+\Delta)
$$

where $\underset{\sim}{\mathrm{FB}}$ is an MXN matrix whose elements denote the amount of each backward wave component to get scattered into each forward wave component. We also note at this point that

$$
\underset{\sim}{B}(z+\Delta)=\underset{\sim}{B}(z)+O(\Delta)
$$

where the 0 function indicates that any additional terms go to zero at least as fast as $\Delta$. Summing the contributions of (21) and (22) with the use of (23) we find

$$
\underset{\sim}{F}(z+\Delta)=\left(\underset{\sim}{I} \mathbb{M}_{\sim}+\underset{\sim}{\mathrm{i}} \delta \Delta\right) \underset{\sim}{F}(z)+\underset{\sim}{i} \underset{\sim}{F B} \underset{\sim}{\Delta B}(z)
$$


Similar reasoning leads to the relation

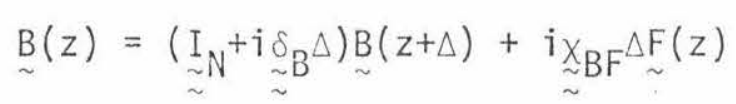

where the new quantities ${\underset{\sim}{B}}_{B}$ and $\underset{\sim}{X_{B F}}$ are defined in analogy with those of (26).

Equations (24) and (25) are now in the form of coupled transmission-1ine equations, a very convenient form in which to apply energy conservation considerations. We accordingly defer our discussion of generalized invariant imbedding for one more sub-section.

2) The Energy Conservation Relations

In his classic work of $1954^{23}$, J. R. Pierce derived, by elementary considerations, a very general set of energy conservation relations applicable to systems expressible as four-port equations. For the system Pierce considered

$$
\left[\begin{array}{l}
F(z+\Delta) \\
B(z)
\end{array}\right]=\left[\begin{array}{ll}
A & B \\
C & D
\end{array}\right]\left[\begin{array}{l}
F(z) \\
B(z+\Delta)
\end{array}\right]
$$

the relations are

$$
\begin{aligned}
& A A^{*}+C C^{*}-1=0 \\
& B B^{*}+D D^{*}-1=0 \\
& A B^{*}+C D^{*}=0
\end{aligned}
$$

where it has been assumed that energy is proportional to the squared modulus of the F and B quantities. We state, without proof, that 
the great generality of the derivation allows us to take the system of (26) and (27) directly over to a matrix case, or more specifically, for the generalized four-port system

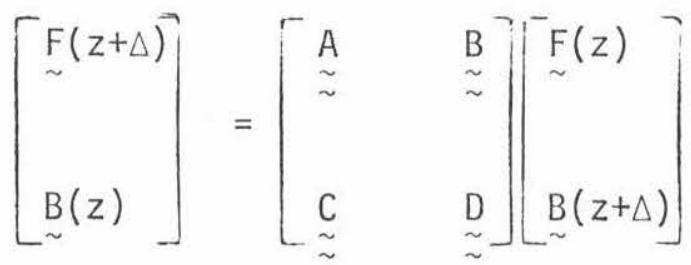

where $\underset{\sim}{F}$ is an $M$ vector and $\underset{\sim}{B}$ an $N$ vector, the energy conservation relations can be written

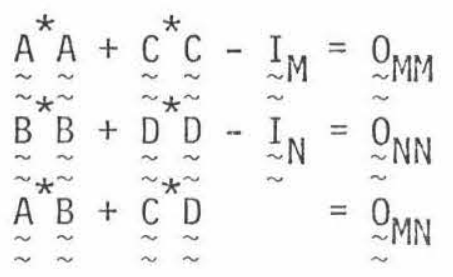

where the definitions are as in (24) and (25), i.e., GMN is the MXN zero matrix, with the exception of the asterisk which here denotes Hermitian transposition.

We note here that the system (28) is of equivalent form to our system of (24) and (25) and therefore that (29) applies as well to (24) and (25). We now want to find what constraints (29) imposes on our propagation matrices if the system of (24) and (25) is to conserve energy. We note, however, that the conditions of (29) need only be satisfied to first order in the parameter $\Delta$ as the system of (24) and (25) is only correct to this order in $\Delta$. Bearing this in 
mind we note that for the identities

$$
\begin{aligned}
& \underset{\sim}{A}=\underset{\sim}{I_{M}}+\underset{\sim}{i} \underset{\sim}{F} \Delta
\end{aligned}
$$

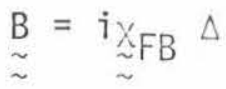

$$
\begin{aligned}
& \underset{\sim}{C}=\underset{\sim}{i \underset{\sim}{X}}{ }_{B F} \Delta \\
& \underset{\sim}{\mathrm{D}}=\underset{\sim}{\mathrm{I}} \mathrm{N}+\underset{\sim}{\mathrm{i}} \delta_{\mathrm{B}} \Delta
\end{aligned}
$$

we find

$$
\begin{aligned}
& \left.\underset{\sim}{A} \underset{\sim}{*} \underset{\sim}{A}=\underset{\sim}{I} M+i \underset{\sim}{\sim} \underset{\sim}{\delta_{F}^{*}} \underset{\sim}{+\delta_{F}}\right)+0\left(\Delta^{2}\right) \\
& \underset{\sim}{B} \underset{\sim}{*} \underset{\sim}{\sim}=0\left(\Delta^{2}\right) \\
& \underset{\sim}{\sim} \underset{\sim}{\sim} \underset{\sim}{\sim}=0\left(\Delta^{2}\right) \\
& \left.\underset{\sim}{D} \underset{\sim}{\sim} \underset{\sim}{\sim}{\underset{\sim}{N}}_{N}+i \Delta \underset{\sim}{\sim} \underset{\sim}{\delta_{B}^{*}} \underset{\sim}{+\delta_{B}}\right)+0\left(\Delta^{2}\right) \\
& \underset{\sim}{A} \underset{\sim}{*} \underset{\sim}{\sim} i \underset{\sim}{\sim} \underset{F B}{ }
\end{aligned}
$$

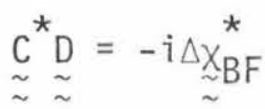

With the substitutions of (31) in (29), we find our energy conservation relations are

$$
\begin{aligned}
& \underset{\sim}{\delta}{ }_{\mathcal{F}}-\underset{\sim}{\delta}{ }^{*}=\underset{\sim}{0} \mathrm{MM} \\
& \underset{\sim}{\delta}-{\underset{\sim}{\delta}}^{\delta^{*}}={\underset{\sim}{\sim}}_{\sim} \mathrm{NN} \\
& \underset{\sim}{\chi_{B F}}-{\underset{\sim}{x}}_{F B}=0_{N M}
\end{aligned}
$$


We find that energy conservation dictates the reality and symmetry of the $\Delta^{\prime} s$ and a Hermitian transpose equality of the $x^{\prime} s$. We will later see that these relations do hold for our coupled-propagation theory derived from the dispersion matrix.

3) Invariant Imbedding of Coupled Equations

We now wish to transform our system of (24) and (25) to a set of differential equations. The change is easily effected as we need only rewrite (24) and (25) in the form

$$
\begin{aligned}
& \frac{\underset{\sim}{F}(z+\Delta)-\underset{\sim}{F}(z)}{\Delta}=i \underset{\sim}{i} \underset{\sim}{F} \underset{\sim}{F}(z)+\underset{\sim}{i \underset{\sim}{F B}} \underset{\sim}{B}(z) \\
& -\frac{\underset{\sim}{B}(z+\Delta)-\underset{\sim}{B}(z)}{\Delta}=i \underset{\sim}{i} \underset{\sim}{B}(z)+\underset{\sim}{i \underset{\sim}{X}} \underset{\sim}{F} \underset{\sim}{F}(z)
\end{aligned}
$$

and take the limit of $\Delta$ going to zero to find

$$
\begin{aligned}
& \frac{\mathrm{dF}_{\sim}(z)}{\mathrm{d} z}=\underset{\sim}{\mathrm{i} \delta_{F}} \underset{\sim}{F}(z)+\underset{\sim}{i \underset{\sim}{\mathrm{F} B}} \underset{\sim}{\mathrm{B}}(\mathrm{z}) \\
& -\frac{\mathrm{dB}(z)}{\mathrm{d} z}=\underset{\sim}{\mathrm{i} \delta_{\mathrm{B}}} \underset{\sim}{\mathrm{B}}(\mathrm{z})+\underset{\sim}{\mathrm{i} \underset{\mathrm{B}}{\mathrm{B}} \boldsymbol{F}} \underset{\sim}{\mathrm{F}}(\mathrm{z})
\end{aligned}
$$

In general our boundary conditions will take the form

$$
\underset{\sim}{F}\left(-\frac{l}{2}\right)=\underset{\sim}{e_{i}} \quad \underset{\sim}{B}\left(\frac{l}{2}\right)=\underset{\sim}{0}
$$

where

$$
e_{i}^{T}=[0,-\cdots, 0,1,0,-\cdots, 0]
$$

corresponding to the situation in figure (4) where only one 
distinguishable wave would be impinging on the slab from the left and no other source in the problem. Such two point boundary value problems can be solved by a straightforward, yet arduous and usually numerical, technique. This technique and its growth in complexity with the order of the system are described in Appendix D. What we wish to do here is to apply invariant imbedding and reduce the reflection coefficient problem to one of the initial value type.

Consider the diagram of figure (5). The diagram is very similar to that of figure (3), the geometry that Stokes $^{9}$ considered, with one basic difference: $\Delta$ is now a continuous and not a discrete, variable. The case here is equivalent to that considered by Schmidt ${ }^{12}$ in which $\Delta$ is at first considered small and later allowed to go to zero. Figure (6) schematically illustrates the procedure of the calculation. In (a) a source is incident on the added thickness of slab. This extra slab immediately backscatters an amount given by the term in $x$ written to the left of the slab, and passes an amount given by the term in $\underset{\sim}{\delta}$ F written to the right of the slab. The term on the left

$$
\text { 1st contribution }=\underset{\sim}{i} \underset{\sim}{x_{B}} \Delta
$$

is seen to directly contribute to the reflection coefficient as that wave is lost to the system. The amount passed, however, is not lost to the system but in fact acts as a source for the original slab problem whose solution we have assumed is known. For this reason, the $\underset{\sim F}{\delta_{F}}$ term returns to our considerations in (b) where the portion of this secondary source 
125.
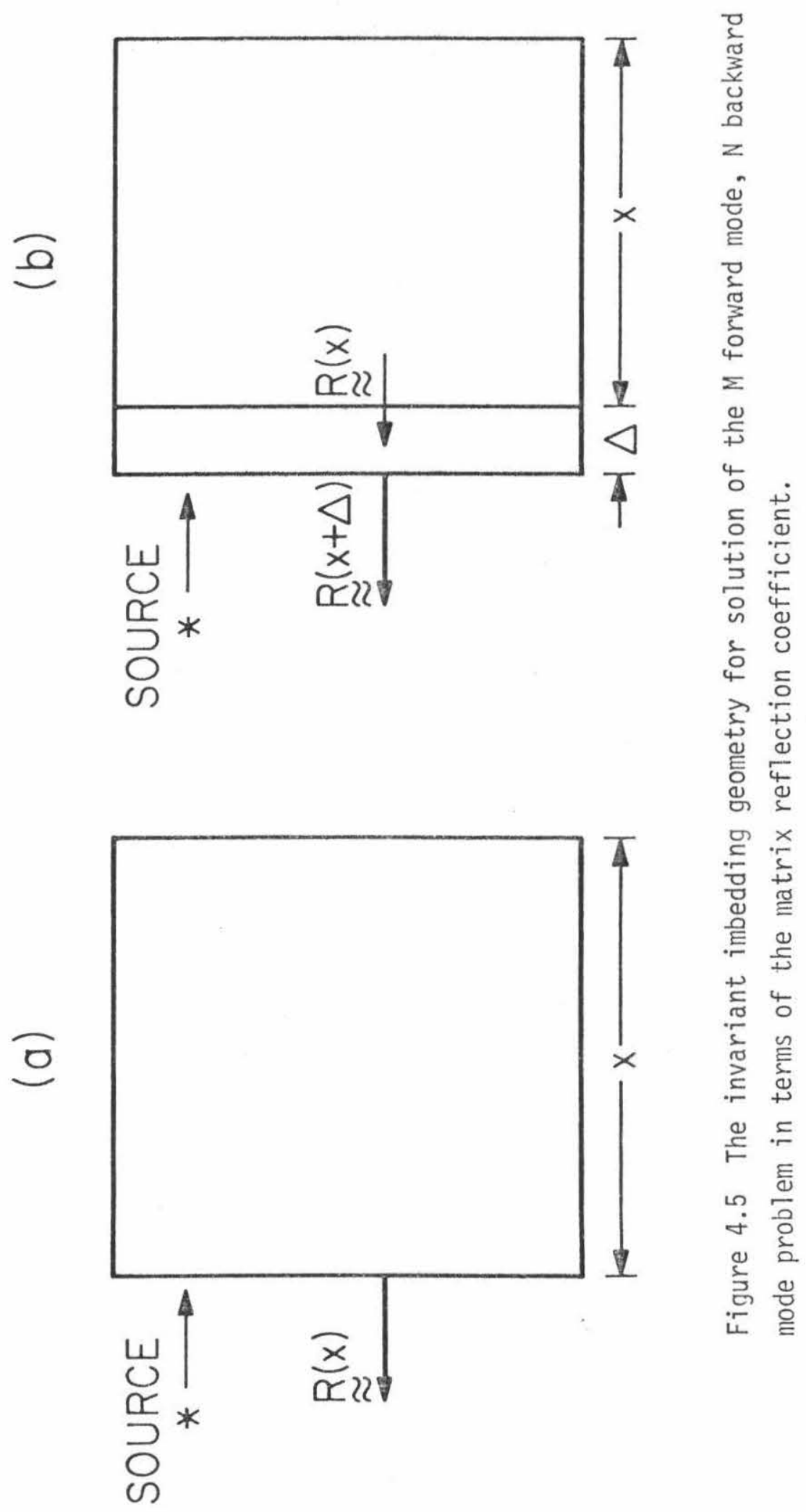


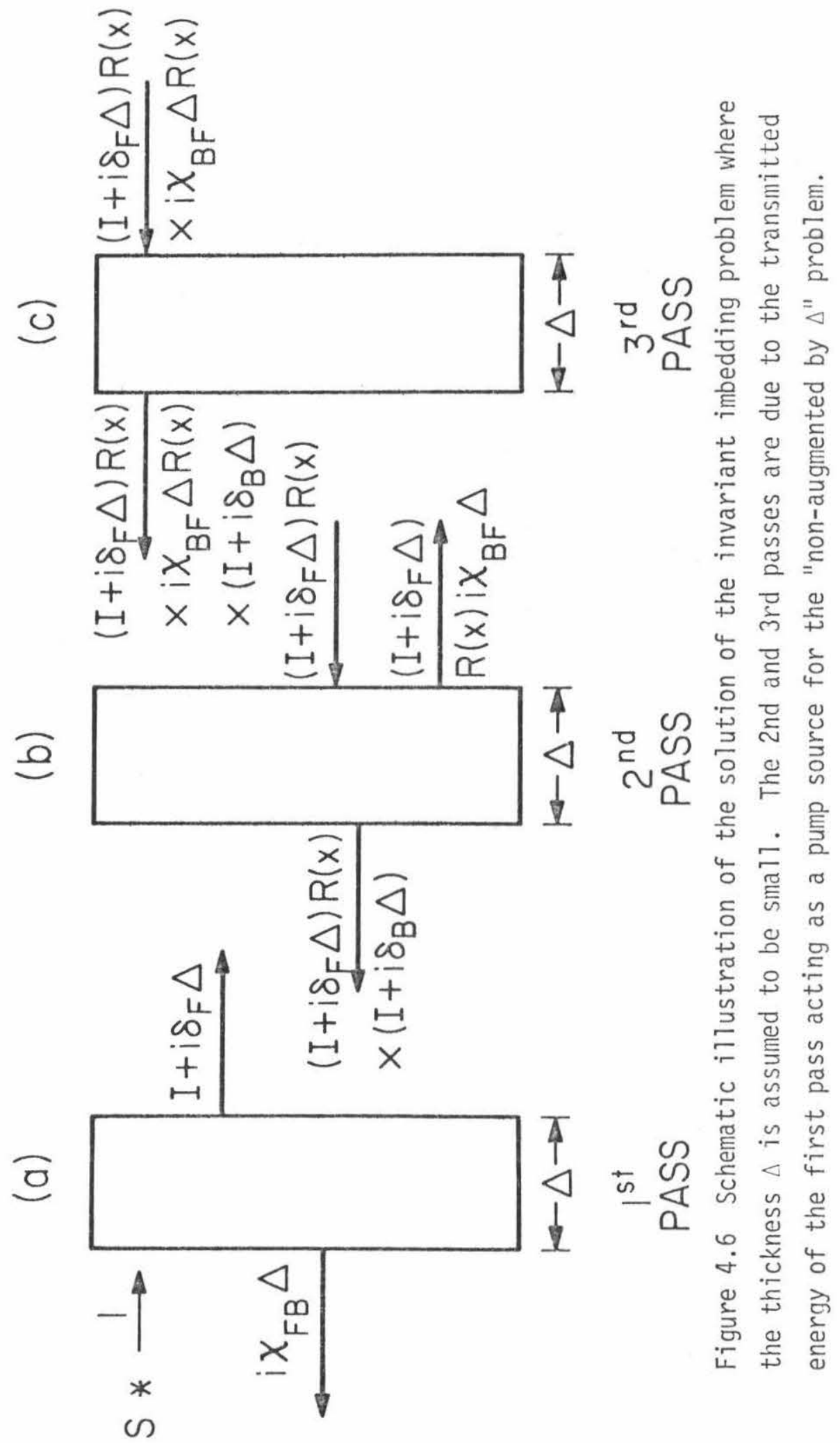




$$
\text { 2nd contribution }=\left(\underset{\sim}{\mathrm{I}} \mathrm{M}+\underset{\sim}{\mathrm{i}}{\underset{\sim}{F}}_{\mathrm{F}} \Delta \underset{\sim}{\mathrm{R}}(\mathrm{z})(\underset{\sim}{\mathrm{I}} \mathrm{N}+\underset{\sim}{\mathrm{i}}{\underset{\sim}{B}} \Delta)\right.
$$

which passes through the extra slab, is seen to directly contribute to our reflection coefficient while the back reflected portion acts as a tertiary source for the original slab. In (c) we note that this tertiary source contributes an amount

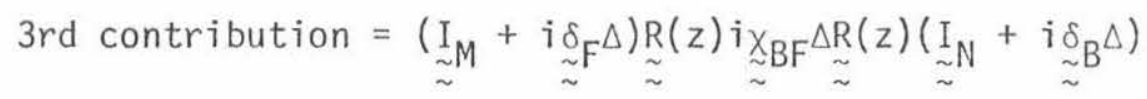

to the reflection. We could continue this summing process ad infinitum, but realize we already have more than we need as we are expanding only to first order in $\Delta$. Ignoring the higher order terms in $\Delta$, we find that our expression can be written in the form

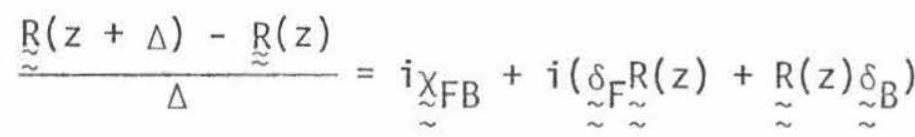

$$
\begin{aligned}
& +i \underset{\sim}{R}(z) \underset{\sim}{\underset{\sim}{X}} \mathrm{BF} \underset{\sim}{R}(z)
\end{aligned}
$$

which in the limit of vanishing $\Delta$ becomes

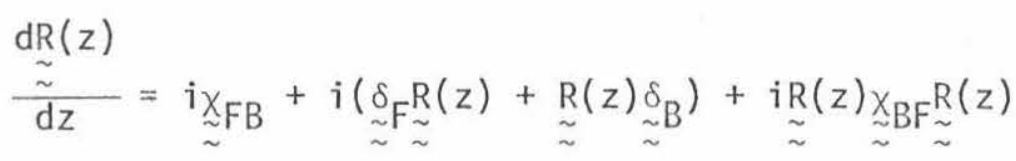

The associated initial condition would be

$$
\underset{\sim}{\mathrm{R}}(\mathrm{z}=0)=\underset{\sim}{\underset{\sim}{\sim} \mathrm{MN}}
$$

if the media to either side of the slab were equivalent.

A few words are necessary here about having a matrix reflection coefficient. In measuring reflection in general we measure at best 
a vector, corresponding to distinguishable backward modes. But here we have derived a matrix reflection. The explanation is simple though. If we consider the $i, j$ component of $R$ we realize it denotes the amount of wave exiting in the $j$ th backward mode, given the $i$ th forward mode was incident. The vector we would therefore measure is

$$
\stackrel{\sim}{r}^{*}=\underset{\sim}{\mathrm{F}_{\text {initial }}^{*}} \underset{\sim}{\mathrm{R}}
$$

where $\underset{\sim}{F_{\text {initial }}}$ should be normalized such that

$$
\underset{\sim \text { initial }}{\mathrm{F}_{\sim}^{*}} \text { initial }=1
$$

Therefore, if we measure only the total reflected energy, the quantity we measure would be

$$
\underset{\sim}{r^{*}} \underset{\sim}{r}=\text { Finitial }_{\sim}^{*} \underset{\sim}{R} \underset{\sim}{R^{*}} \underset{\sim}{\text { Finitial }}
$$

A few words are necessary here about what we have actually achieved. In (41) we have a non-linear differential equation for a matrix, a very complicated system to solve analytically. The achievement however lies in the fact that (41) has constant coefficients in the cases which we will presently consider. This is an extremely desirable improvement over the electromagnetic Riccati equation as we are no longer limited to some number of periods inside the medium in a numerical solution, as the addition of more periods simply causes the redefinition of one of the constant coefficients of a numerically stable system. We have succeeded in applying a perturbation theory to our original problem, which both simplifies the 


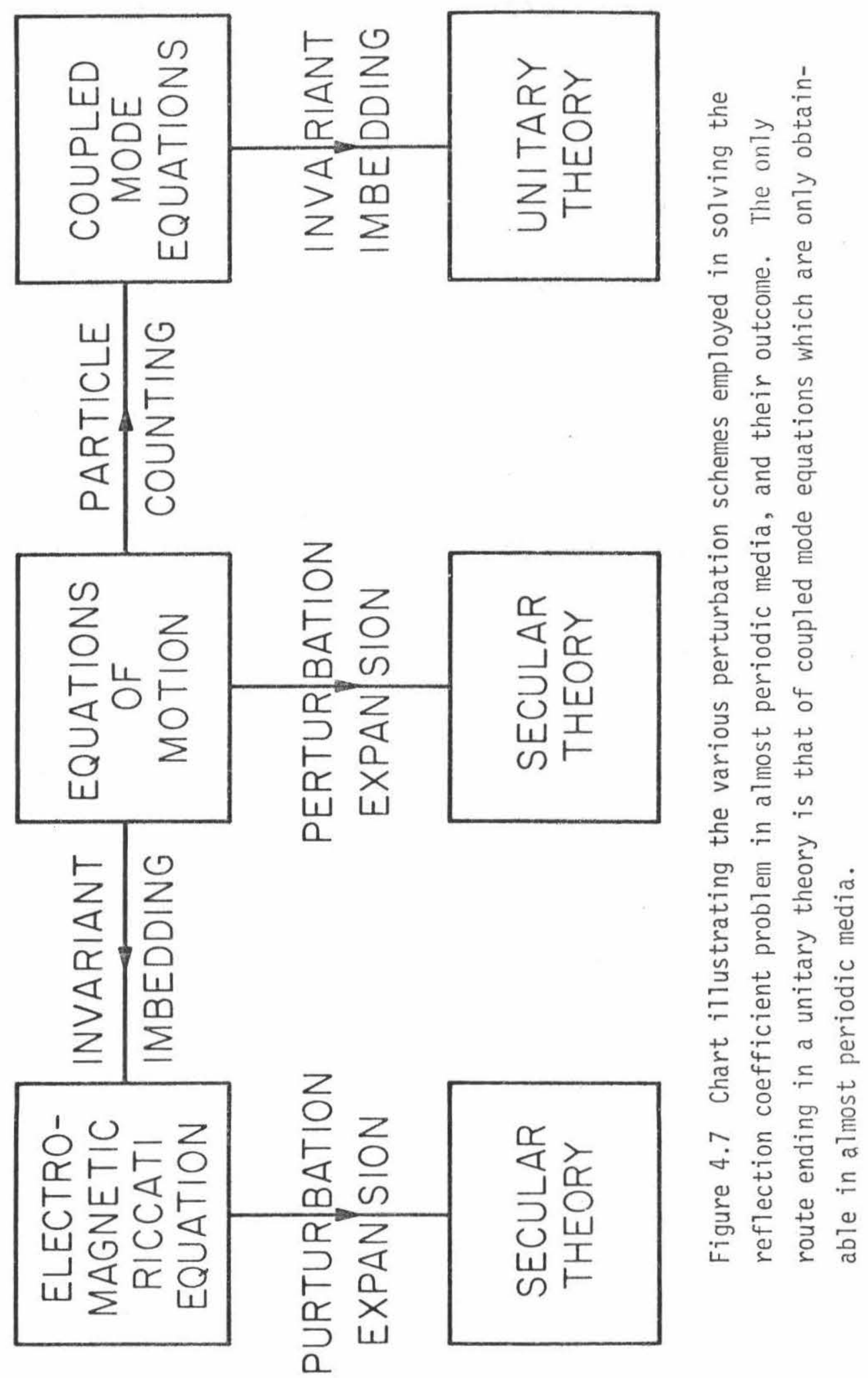


method of solution and preserves the unitarity of the theory. We schematically illustrate our approach and the approaches that fail in figure 7. More will be said on this in the next section where we wil1 apply (41) to some systems of Chapter III.

D) Riccati Equations, Brillouin Diagrams and Dispersion Matrices

1) Single Harmonic Perturbation

Before considering the case of bandcoalescence, we wish to apply our technique to the simplest problem we know, that of a single harmonic perturbation. The schematic Brillouin diagram for this problem would be

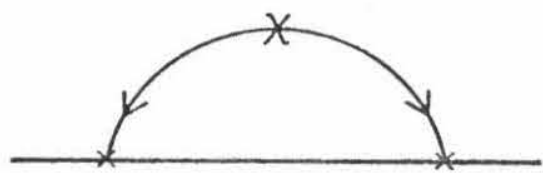

representing the set of equations

$$
\begin{array}{r}
\frac{d F}{d z}+i \delta F=i X B \\
-\frac{d B}{d z}+i \delta B=i X F
\end{array}
$$

where

$$
\begin{aligned}
& \delta=\Delta k \\
& x=\frac{\eta \kappa}{8}
\end{aligned}
$$

where the notations of Chapter III have been used. We note here that indeed the system of (47) satisfies the criteria of (32). To find the reflection coefficient we can immediately employ the Riccati equation (41), which now takes the simple form 


$$
\frac{d R}{d z}=i x\left(1+R^{2}\right)+2 i \delta R
$$

where the identifications of (48) apply. Equation (49) has constant coefficients and can therefore be solved by quadrature

$$
\int_{0}^{R} \frac{d r}{i x\left(1+R^{2}\right)+2 i \delta R}=\int_{0}^{z} d z .
$$

The integral on the left hand side is available in Gradshtein and Ryzhik's manual ${ }^{24}$ and, with some algebraic manipulation, the resulting equation can be inverted to yield

$$
R(z)=\frac{i x}{D \operatorname{coth}(D z)-i \delta}
$$

where

$$
D=\sqrt{\delta^{2}-x^{2}}
$$

The result (51) is a familiar one from the 1 iterature $25-26$ where it has been obtained by solving the coupled-mode two-point boundary value problem. It has also been shown elsewhere $25-26$ that (51) gives a very good prediction of experimental results, and further that the half-width of the main lobe is very close to the width of the bandgap. In Appendix $E$ this result is compared with that of the exact Riccati equation.

2) The Almost Periodic $\Delta K \rightarrow 0$ Solution.

We next reconsider a problem from the last section of the previous chapter, which can be represented by 


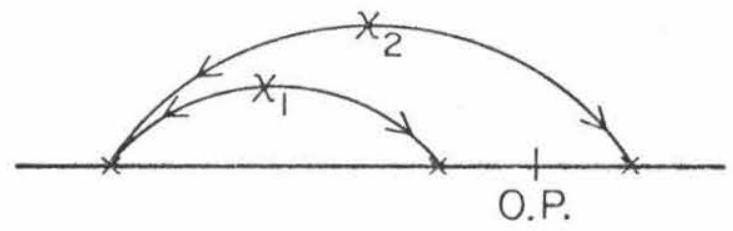

where 0.P. abreviates operating point and is used to indicate that we are expanding about this point. The schematic (53) represents the system

$$
\begin{aligned}
& \frac{d F}{d z}-i \delta_{0} F=i\left(x_{1} B_{1}+x_{2} B_{2}\right) \\
& -\frac{d B_{1}}{d z}-i \delta_{+} B_{1}=i x_{1} F \\
& -\frac{d B_{2}}{d z}-i \delta_{-} B_{2}=i x_{2} F
\end{aligned}
$$

where

$$
\begin{aligned}
& \delta_{0}=\Delta k \\
& \delta_{ \pm}=\Delta k \pm \Delta
\end{aligned}
$$

and

$$
\begin{aligned}
& x_{1}=\frac{n_{1} \bar{k}}{8} \\
& x_{2}=\frac{n_{2} \bar{k}}{8}
\end{aligned}
$$

and

$$
\bar{\kappa}=\frac{\kappa_{1}+\kappa_{2}}{2}
$$

The notation employed here is just that of the last section of 
Chapter III except for $\Delta$ replacing $\delta$ of Chapter III. Again we can directly employ the Riccati equation of (41), providing we make the identifications

$$
\begin{aligned}
& \underset{\sim}{\delta_{F}}=\delta_{0} \\
& \underset{\sim}{\delta_{B}}=\left[\begin{array}{ll}
\delta_{+} & \\
0 & \delta_{-}
\end{array}\right]
\end{aligned}
$$

and

$$
\begin{aligned}
& \underset{\sim}{x_{F B}}=\left[\begin{array}{l}
x_{1} \\
x_{2}
\end{array}\right] \\
& \underset{\sim}{x_{B F}}=\left[x_{1} x_{2}\right]
\end{aligned}
$$

We note at this point that (58) and (59) do indeed satisfy (32), the Pierce energy-conservation criteria. The resulting set of reflection coefficient equations can now be written out explicitly and are found to be

$$
\begin{aligned}
& \frac{d R_{1}}{d z}=i x_{1}\left(1+R_{1}^{2}\right)+i\left(\delta_{0}+\delta_{+}\right) R_{1}+i x_{2} R_{1} R_{2} \\
& \frac{d R_{2}}{d z}=i x_{2}\left(1+R_{2}^{2}\right)+i\left(\delta_{0}+\delta_{-}\right) R_{2}+i x_{1} R_{1} R_{2}
\end{aligned}
$$

We are not in so fortunate a position with equation (60) as we were with equation (49) as although (60) has constant coefficients, it is no longer integrable by simple quadratures. However we programmed the system of (60) in a program called "Criccatti" and will 
now present some of the results.

Figures (8) and (9) represent solutions of the system (60) for parameters given by

$$
\begin{aligned}
& \overline{k_{l}}=8.10^{3} \\
& \eta_{1}=\sqrt{2} 10^{-3} \quad n_{2}=\sqrt{2} 10^{-3} \\
& x_{1} l=\sqrt{2} \quad x_{2} l=\sqrt{2} \\
& \delta l=\left\{7 . \delta_{c}, 4 . \delta_{c}\right\}=\left\{7 . \sqrt{x_{1}{ }^{2}+x_{2}^{2}}, 4 \cdot \sqrt{x_{1}^{2}+x_{2}^{2}}\right\}
\end{aligned}
$$

i.e., the widely spaced perturbation limit. It is notable that in this limit the results appear much as the result of two independent single perturbation solutions ( $c f$. APPENDIX E) patched together might appear. From the considerations of Chapter III we would assume this would be the case.

In figures (10)-(13) we plot a sequence of graphs for constant

$$
x_{1} l=x_{2} l=\sqrt{2}
$$

while varying $\delta l$ through the values

$$
\begin{array}{lll}
\delta l=2 . \delta_{c}=2 . v^{\prime} x_{1}^{2}+x_{2}^{2} & \text { figure } 10 & \text { (a) } \\
\delta l=1.5 \delta_{c}=1.5 \sqrt{x_{1}^{2}+x_{2}^{2}} & \text { figure } 11 \quad \text { (b) } \\
\delta l=1 . \delta_{c}=1 . \sqrt{x_{1}^{2}+x_{2}^{2}} & \text { figure } 12 \quad \text { (c) } \\
\delta l=.5 \delta_{c}=.5 \sqrt{x_{1}^{2}+x_{2}^{2}} & \text { figure } 13, \text { (d) }
\end{array}
$$

i.e., the closely spaced perturbation limit. The results are just what we would have expected qualitatively from the Brillouin diagram 


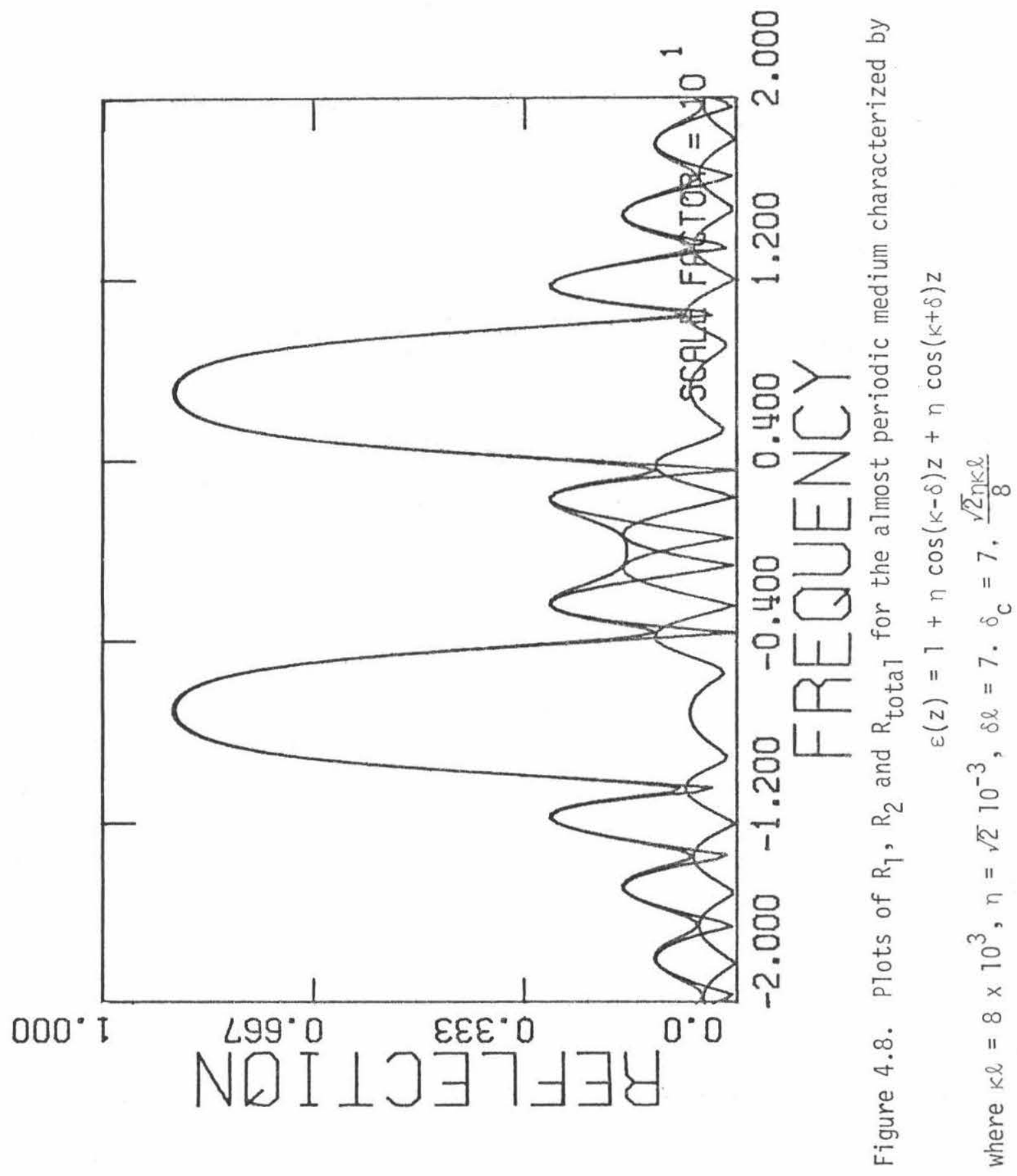


136.

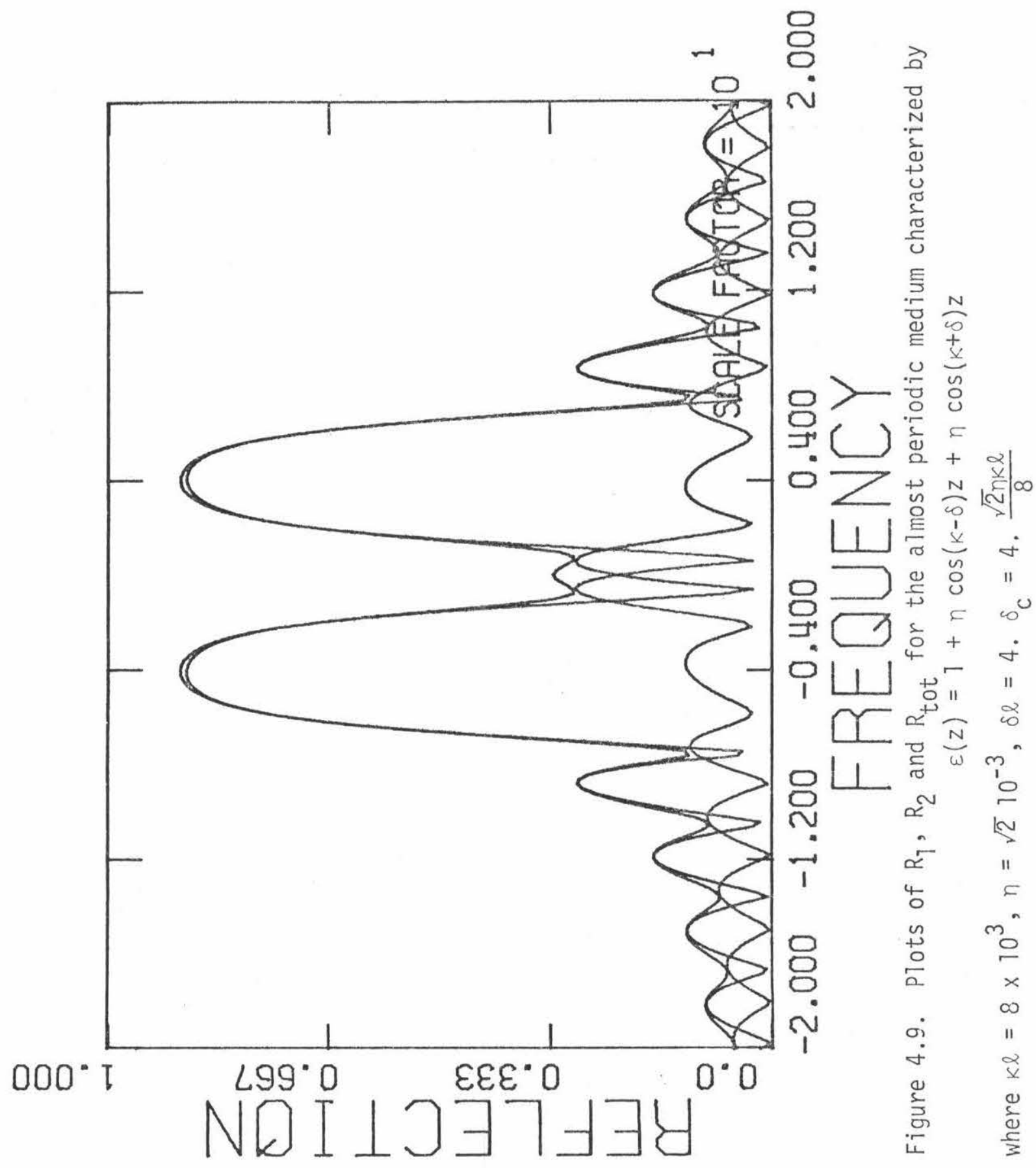


137.

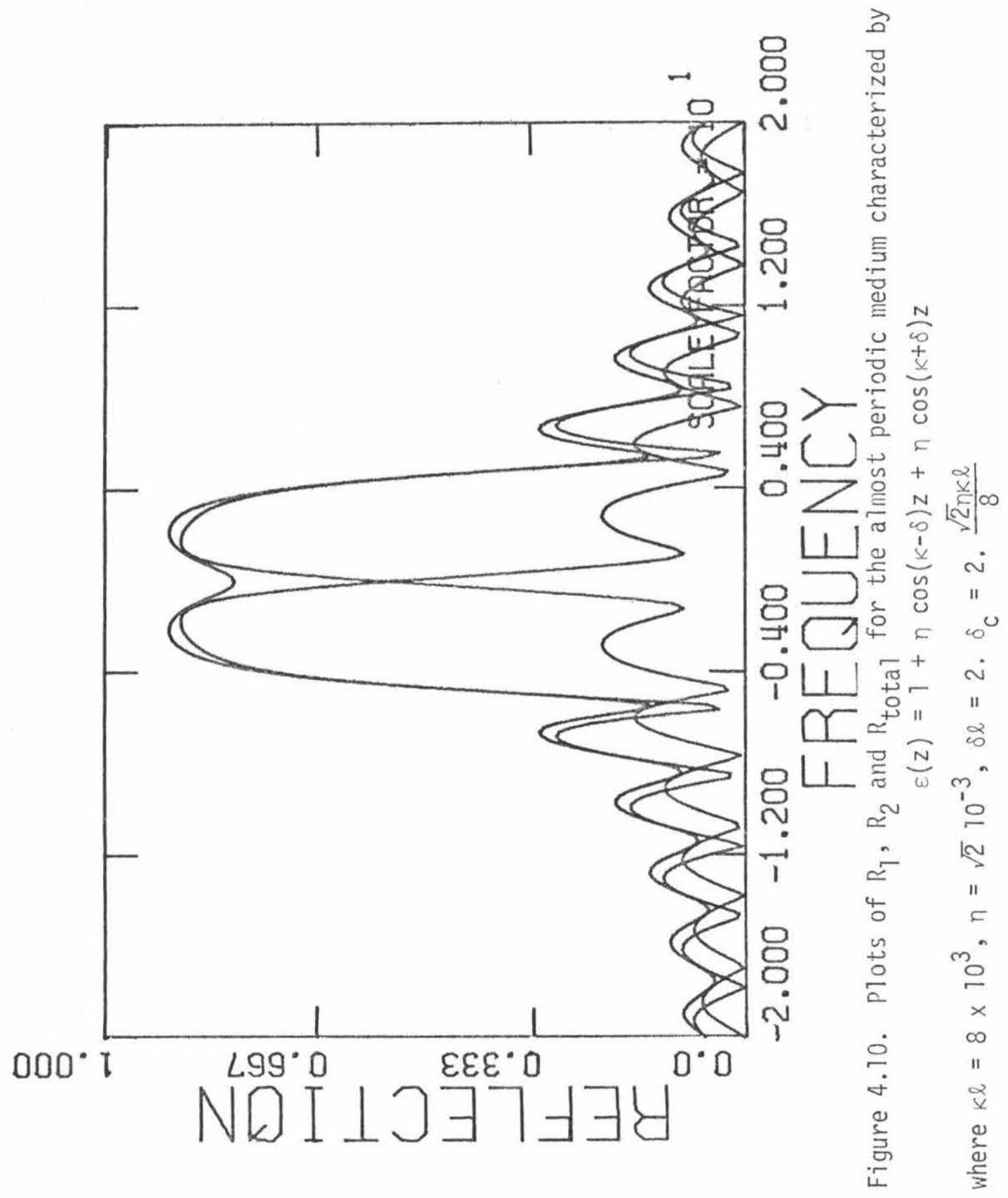




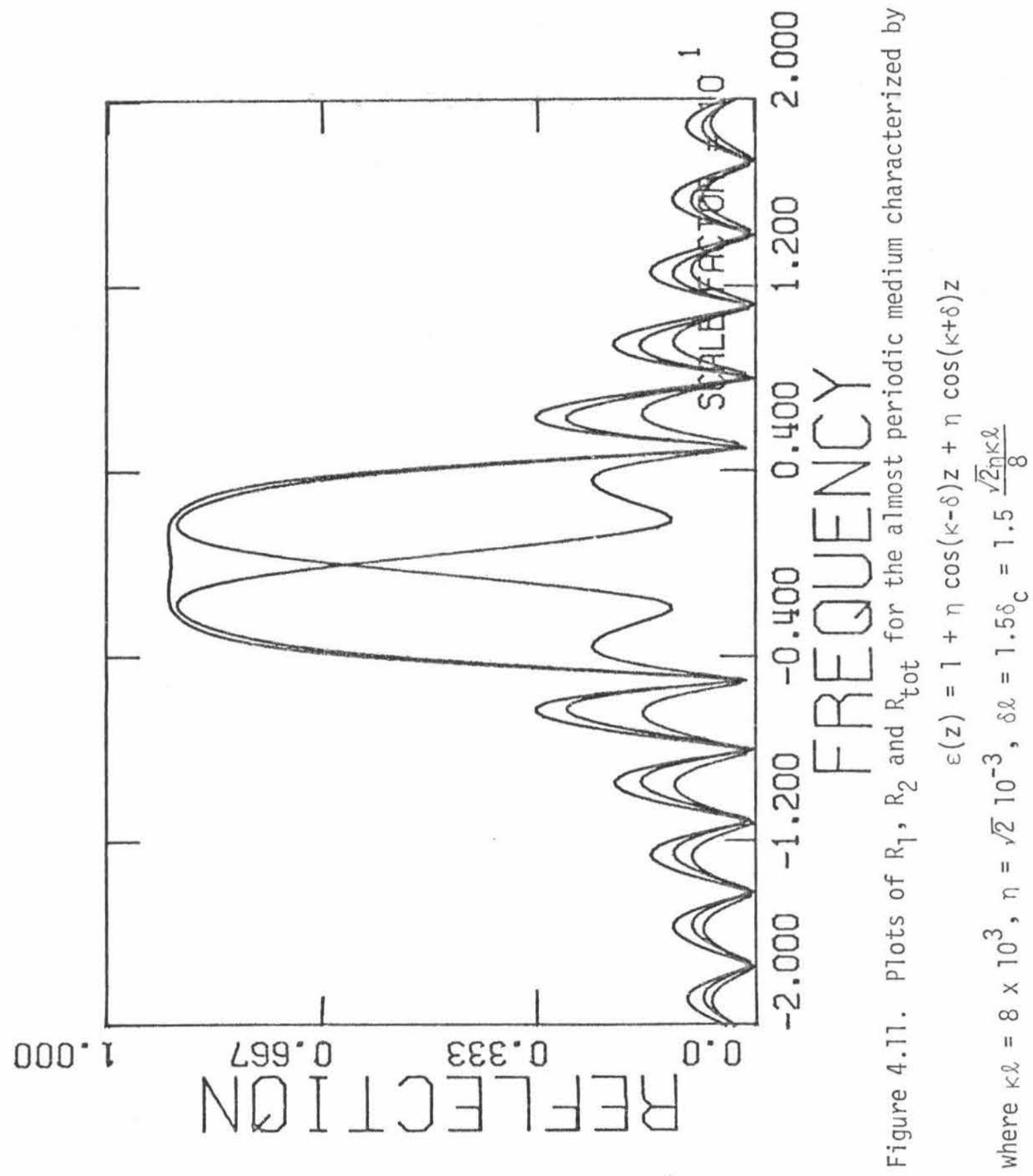




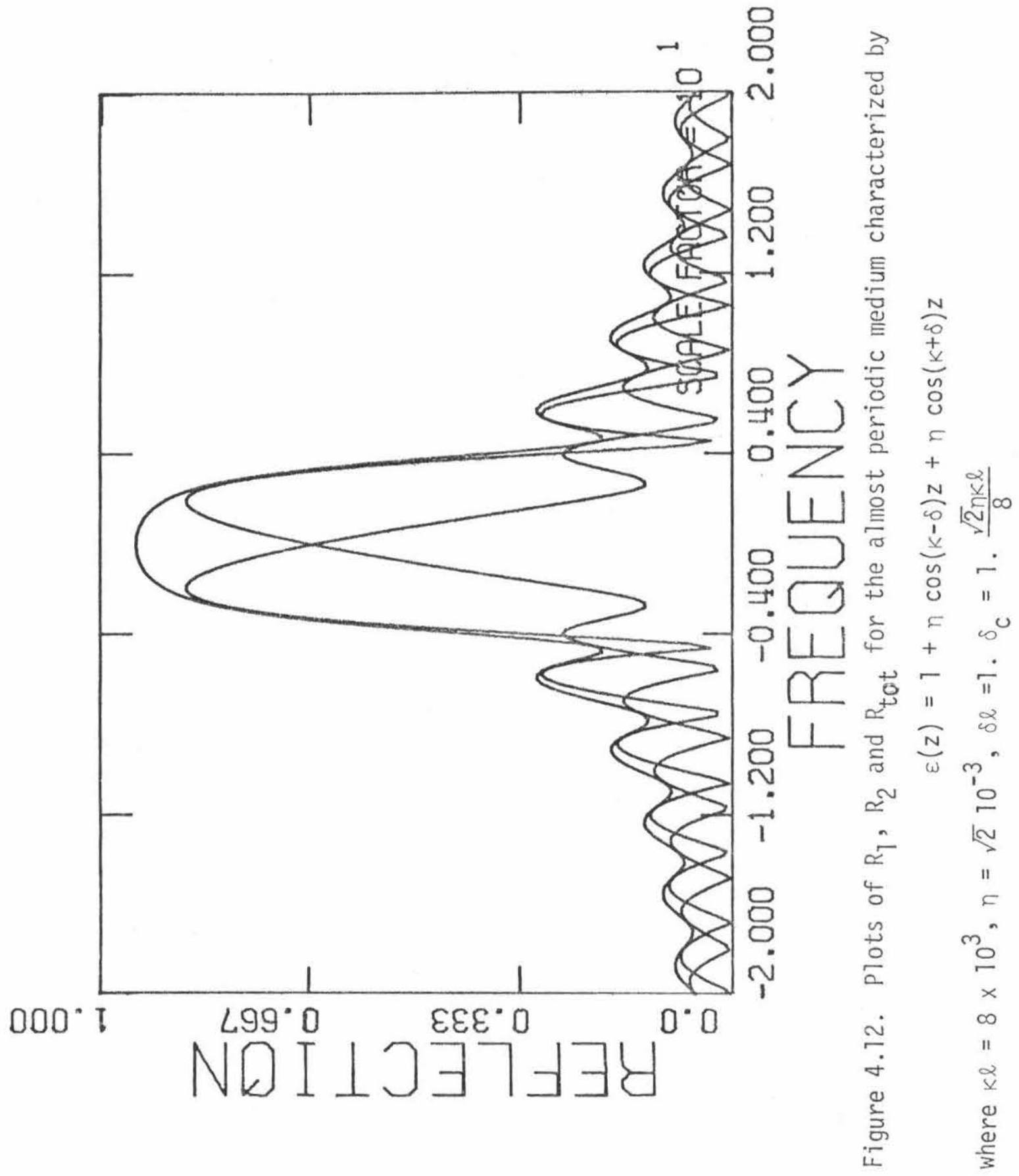




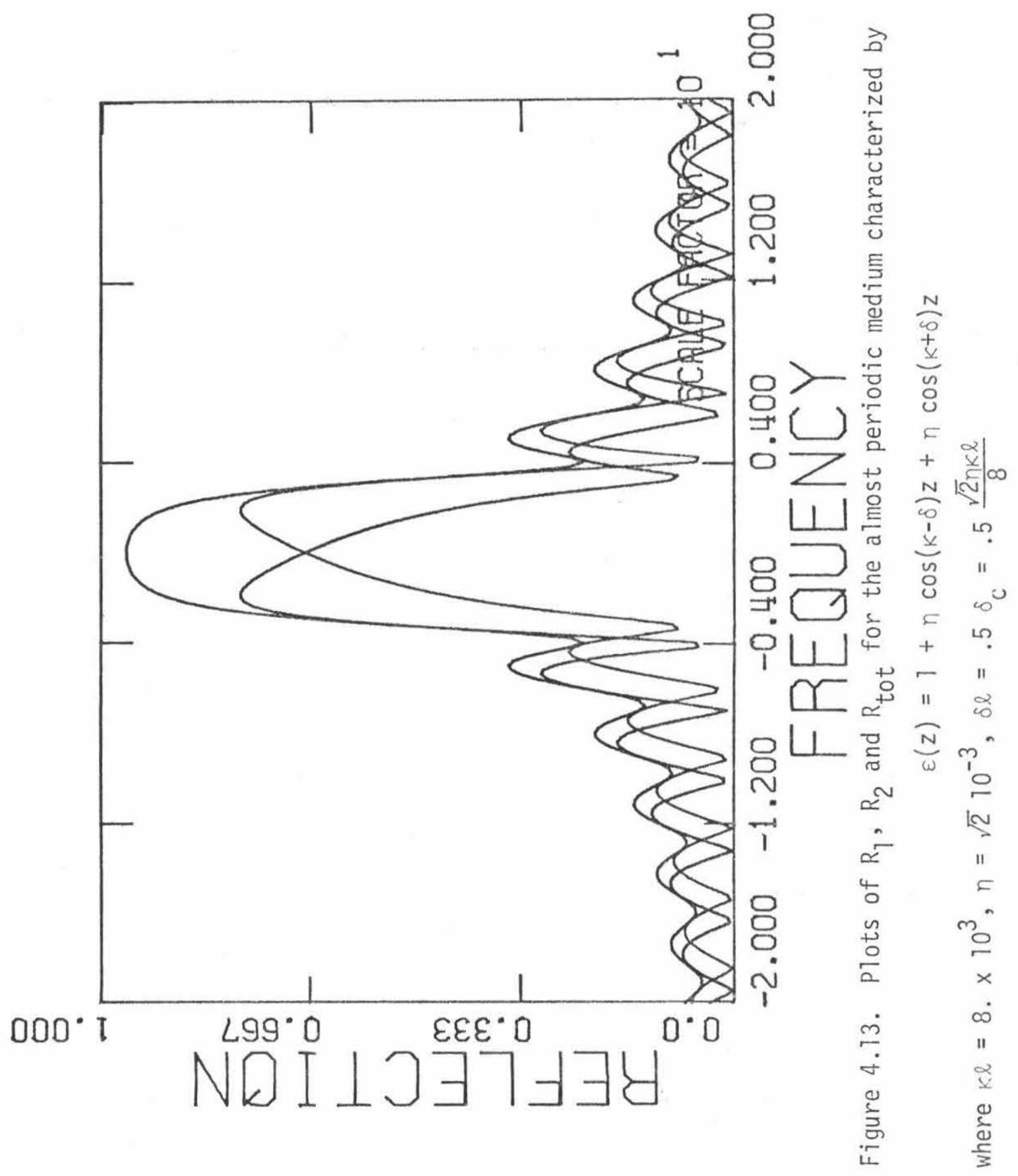


plots of the last section of Chapter III (figures (20)-(23) of Chapter III). We also note that in figure (14) the total reflection coefficient appears to be just that of the root mean square $x$ defined, as in Chapter III, by

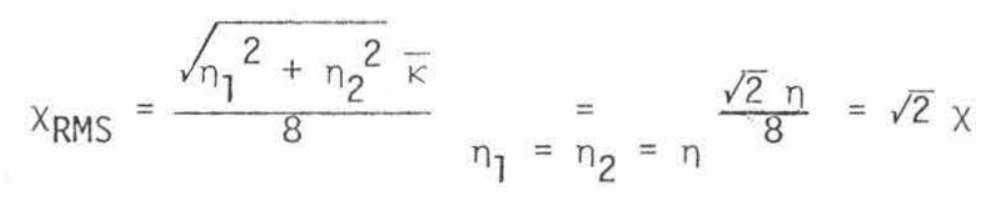

We can illustrate this effect analytically by the following argument. If we take

$$
\begin{aligned}
& x_{1}=x_{2}=x \\
& \delta_{+}=\delta_{0}=\delta_{-}=\delta
\end{aligned}
$$

then we see that

$$
R_{1}=R_{2}=R / \sqrt{2}
$$

where $R$ denotes the total reflection coefficient which, from (45), is defined as

$$
|R|=\sqrt{\left|R_{1}\right|^{2}+\left|R_{2}\right|^{2}}
$$

We now write $(60)$ in the form

$$
\begin{aligned}
& \frac{d R_{1}}{d z}=i \times\left(1+R_{1}^{2}\right)+i \times R_{1} R_{2}+2 i \delta R_{1} \\
& \frac{d R_{2}}{d z}=i \times\left(1+R_{2}^{2}\right)+i \times R_{1} R_{2}+2 i \delta R_{2}
\end{aligned}
$$

Now, substituting (66) in (68), we find the single equation

$$
\frac{d R}{d z}=\sqrt{2} x\left(1+R^{2}\right)+2 i \delta R=x_{R M S}\left(1+R^{2}\right)+2 i \delta R
$$


142.

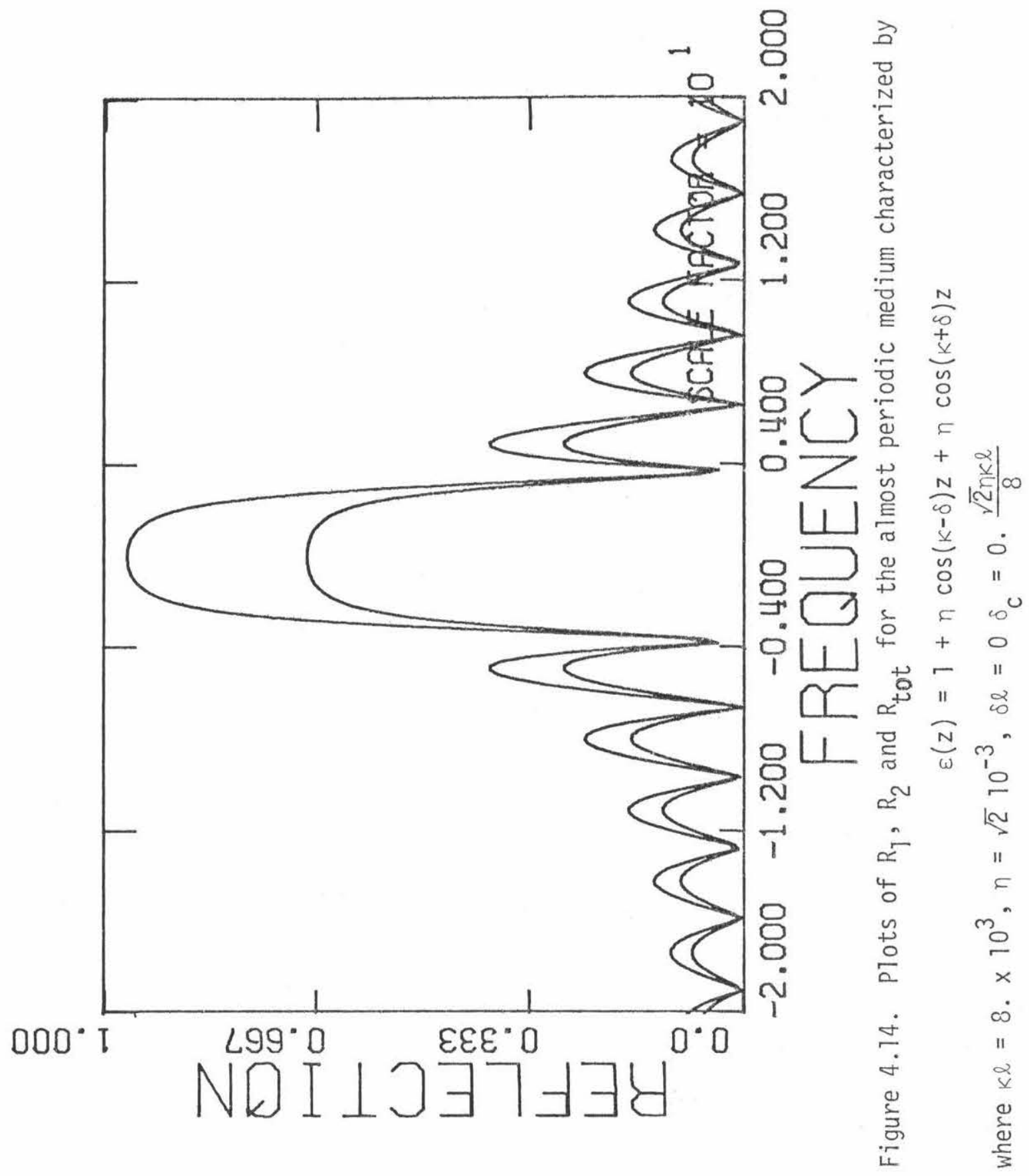


But (69) is equivalent to (49) with $x$ replaced by $x_{\text {RMS }}$ and we have illustrated that our system (60) has the correct limit in the sense that it agrees with the result of the two-point boundary value problem, which in turn is shown to closely agree with the electromagnetic Riccati equation in Appendix E.

As the system (60) has the correct limits for $\Delta$ becoming large and $\Delta$ going to zero, it seems a safe assumption to consider (60) the result of a correct perturbation theory. Also the close analogy between the results of the reflection coefficients and the Brillouin diagrams generated by our approach also tends to vindicate our assumption that the Brillouin diagram does contain much information about a medium's properties. As the reflection coefficient and Brillouin diagram calculations come from an equivalent set of equations we therefore feel we can consider either of them basic quantities.

3) The Periodic Media Small $\Delta \kappa$ Limit

We note here that the system (34), assuming a harmonic spatial dependence with characteristic exponent $\Delta \beta$, can be written in the form

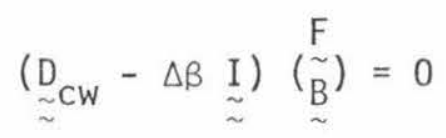

with the characteristic equation

$$
\operatorname{det} \underset{\sim}{(D \mathrm{CW}}-\Delta \beta \underset{\sim}{\mathrm{I}})=0
$$

which is equivalent to equation (65) of Chapter III. We also note 
that equation (65) of Chapter III satisfies the Pierce energyconservation criteria (Eqs. (32) of this chapter). The point of writing (70) and (71) was to point out that equation (41) is simply an imbedding of a problem we have essentially solved in Chapter III; that is to say, having been given the Brillouin diagrain we have a good idea of the structure of the reflection coefficient. We therefore, without further reflection coefficient calculations employing (41), will proceed to compare the almost periodic and periodic cases in a qualitative sense.

Recalling figures (18), (19) and (24)-(28) of Chapter III and employing the results of this chapter, we would conclude that the reflection coefficient of periodic media in the small $\Delta k$ limit is a complicated, broad-band structure, probably containing many dips and peaks. An example is plotted in figure (15), employing the exact Riccati equation, and our conclusions are well borne out. The basic point is that the periodic case is considerably more complicated than the almost periodic case. We now will attempt to give physical meaning to this distinction.

Consider the periodic or almost periodic dielectric constant given by

$$
\varepsilon(z)=\varepsilon_{r}[1+\eta(\cos \kappa z+\cos ((\kappa+\Delta \kappa) z)]
$$

Using a well known trigonometric identity we can write the second bracketed term as

$$
\cos \kappa z+\cos ((\kappa+\Delta \kappa) z)=2 \cos \left(\frac{\Delta \kappa z}{2}\right) \cos \left(\left(\kappa+\frac{\Delta \kappa}{2}\right) z\right)
$$




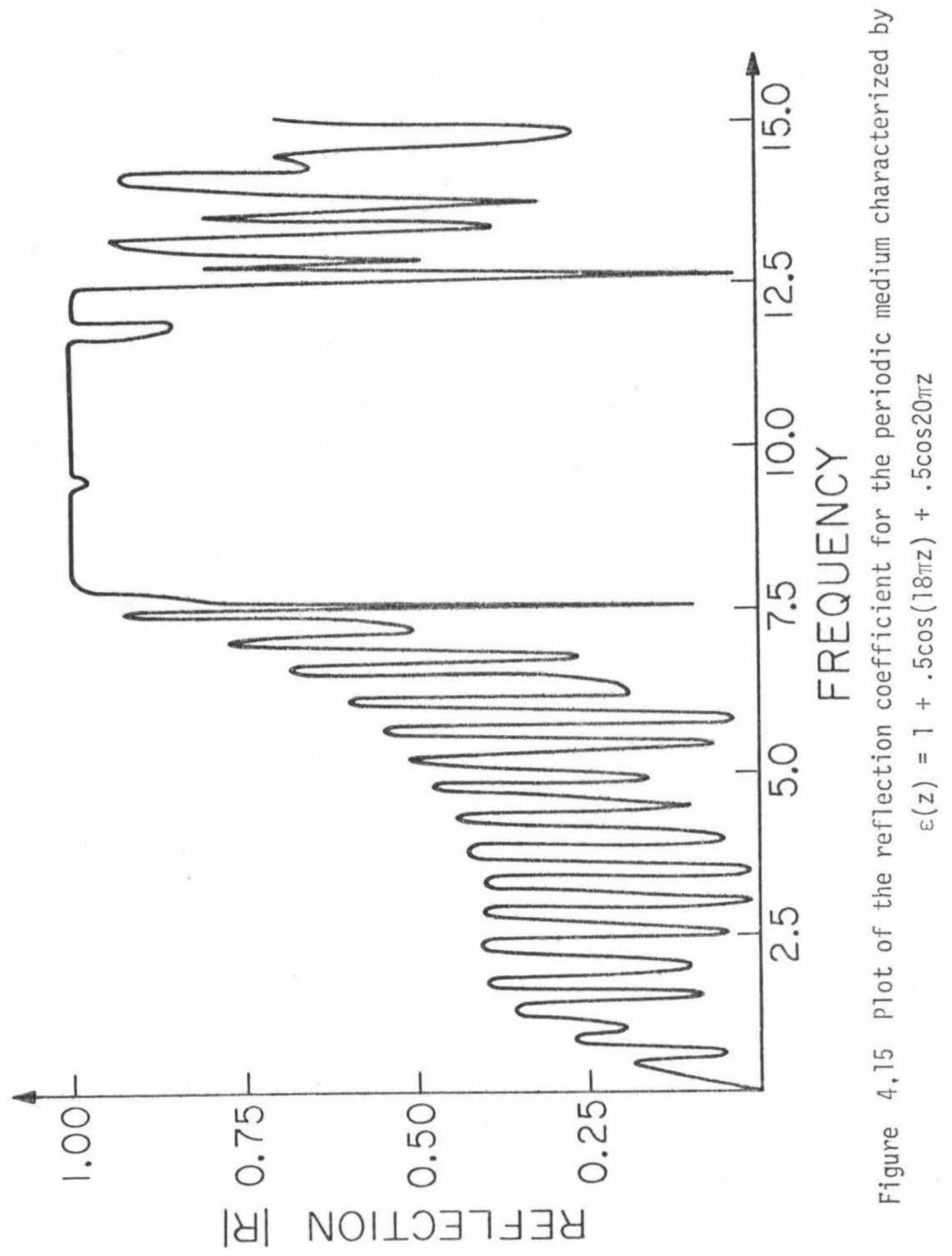


The function (72) is sketched for the periodic and almost periodic cases in figure (16).

To proceed we must first accept the principle of local reflection. The idea behind it is that a wave is strongly reflected by portions of a medium which satisfy the Bragg relation but that the wave is not very much affected by other parts of the medium. Now with reference to figure (16), we notice that the "wiggles" of (72) become more closely spaced near the relative nulls of the wave packet. This packing phenomena is caused by the slope of the modulating wave packet increasing sinusoidally to a maximum at its nulls. The unperturbed carrier peaks would be equally spaced without the wave packet, but in the presence of this modulation, the peaks are squashed inward toward the wave packet maximum at a rate which increases as the slope of the wave packet, as the tips of the unmodulated peaks are being multiplied by a value of the modulation function which is smaller than that which is multiplying the perturbed peak location. The gross effect of the varying peak spacing is that along the z-axis different Bragg conditions are satisfied at different locations. This means that waves of different frequencies are reflected from different positions and one would expect a broadening of the reflected spectrum. But the spectral broadening will only occur in the periodic case as in the periodic case the wave is successively reflected nearly in phase by each of the carrier humps, causing a cumulative effect. This is not so in the almost 

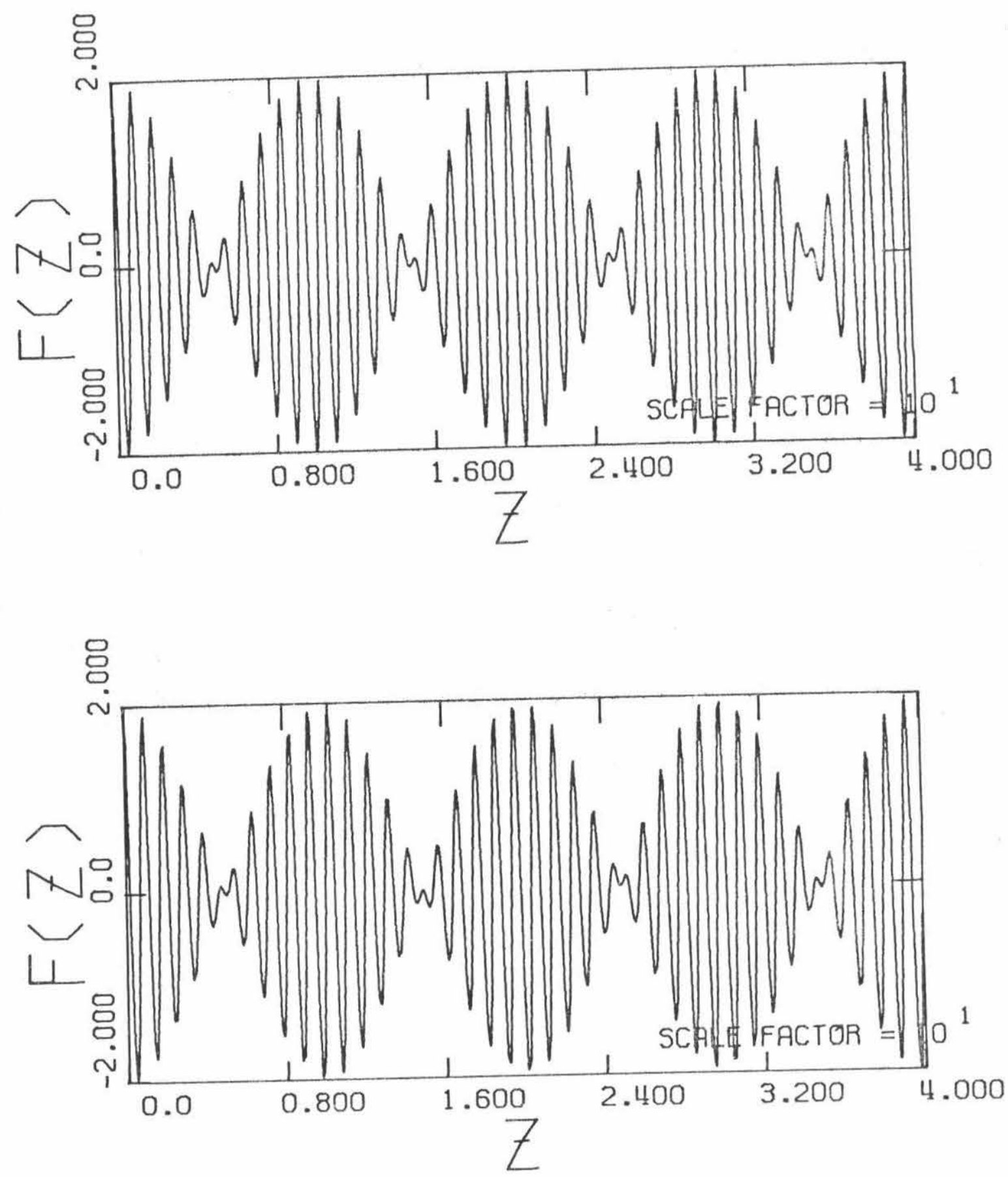

Figure 4.16. a) Plot of the periodic function

$$
f(z)=\cos (1.9 \pi z)+\cos (2.1 \pi z)
$$

b) Plot of the almost periodic function

$$
f(z)=\cos \left(\frac{1.9 \sqrt{3}}{1.7} \pi z\right)+\cos \left(\frac{2 \cdot 1 \sqrt{2}}{1 \cdot 39} \pi z\right)
$$


periodic case as the phasing of the "wiggles" within each carrier hump is essentially random and no cumulative effect occurs, other than that due to the separate periods of (72). This effect, however, will disappear gradually as $\Delta k$ is increased as the number of carrier humps per unit length decreases. We now can see a reason for the behavior we have discovered in this report. In the large $\Delta k 1$ imit the two-tone periodic and almost periodic theories should appear identical; however, this identity will disappear as the frequencies of the two perturbations approach a central limit. 


\section{Bibliography for Chapter Four}

1. Morse, P. M. and H. Feshbach, Methods of Theoretical Physics, Vol. 2 (New York: McGraw-Hill Book Co., 1953).

2. The first term of the series is generally called the Born approximation. See: Schiff, L.I., Quantum Mechanics (New York: McGraw-Hill Book Co., 1968), 3rd edition.

3. Although the series solution to integral equations is generally attributed to Neumann (See, for instance: (Mathews, J. and R. Walker, Mathematical Methods of Physics (Menlo Park, Calif.: W. A. Benjamin, Inc., 1970), 2nd edition or Stakgold, I., Boundary Value Problems of Mathematical Physics, Vol. 1 (New York: The Macmillan Co., 1967).), it appears to actually have been originated by Lioville. See: Whittaker, E. T. and G. N. Watson, A Course of Modern Analysis (London: Cambridge Univ. Press, Fourth Edition 1927, reprinted - 1973). See page 221.

4. The Fourier transform property of the first-order reflection coefficient can be deduced from the Bremmer series. See, for example: Bellman, R. and F. M. Wing, An Introduction to Invariant Imbedding (New York: J. Wiley and Sons, 1975) or K1 ine, M. (Ed.), The Theory of Electromagnetic Waves (New York: Dover Pub., Inc., 1951). See pages $169-180$.

5. The result is well-known. See, for example: Jaggard, D. L., Caltech Antenna Laboratory Report No. 75, 1976. See pages 127-129.

6. For a more precise definition of this terminology, see, for 
instance: Nayfeh, A. H., Perturbation Methods (New York: J. Wiley and Sons, 1973).

7. The two most commonly used renormalization techniques are that of Fredholm and Rytov. For the Fredholm series, see the first reference cited in (3), and for the Rytov series, see the reference cited in (6).

8. Some discussion of the Fredholm series' applicability is contained in reference (1). The accuracy of the Rytov series relative to the Born approximation is discussed in: Sancer, M. and A. Varvatsis, Proc. IEEE 58, 140-141 (1970).

9. Stokes, G. G., Mathematical and Physical Papers, Vol. 4 (London: Cambridge Univ. Press, 1904). See pages 145-156. Note that this reference is a reprint of: Stokes, G. G., Proc. Roy. Soc. (January 23,1862 ).

10. Rayleigh, Lord, Proc. Roy. Soc. A86, 208-226 (1912).

11. Rayleigh, Lord, Proc. Roy. Soc. A93, 565-573 (1917).

12. Schmidt, H. W., Ann. Phys. (Germany) 23, 671 (1907).

13. Ambartsumian, V. A., Dok1ady 38, 229-232 (1943).

14. Ambartsumian,V. A., Theoretical Astrophysics (New York: Pergamon Press, 1958).

15. See, for example: Papas, C. H., Caltech Antenna Laboratory Note, March, 1954.

16. Chandresekhar, S., Radiative Transfer (New York: Dover Pub., Inc., 1960) .

17. See Chapter 12 (authored by Redheffer) in Modern Mathematics for 
the Engineer, edited by E. F. Beckenbach (New York: McGraw-Hill Book Co., 1961), 2nd edition.

18. Specifically, we refer to the reference 1 ists in the first reference of (4). Another useful reference is: Adams, R. N. and E. D. Denman, Wave Propagation and Turbulent Media (New York: American Elsevier, 1966).

19. The first reference of (4).

20. Maxwe11, J. C., A Treatise on Electricity and Magnetism (New York: Dover Pub., Inc., 1954). This is a reprint of the first edition of 1873.

21. Born, M. and E. Wolf, Principles of Optics (New York: Pergamon Press, 1975). See page 324.

22. A good reference is: Watson, G. N., A Treatise on the Theory of Bessel Functions (London: Cambridge Univ. Press, 1966), 2nd edition. See section 4.2 .

23. Pierce, J. R., J.A.P. 25, 179-183 (1954).

24. Gradshtein, I. S. and I. M. Ryzhik, Tables of Integrals, Series and Products (New York: Academic Press, 1965).

25. Yariv, A., Introduction to Optical Electronics (New York: Holt, Rinehart and Winston, 1976).

26. Yariv, A., Quantum Electronics (New York: J. Wiley and Sons, 1975). 
Chapter V

Conclusions

\begin{abstract}
A) Relationship of the Brillouin Diagram to the Reflection Coefficient
\end{abstract}

In Appendix $\mathrm{E}$ of this report the close agreement of the exact Riccati equation and the coupled mode two-point boundary value problem is illustrated. This agreement had previously been pointed out elsewhere. I In Chapter IV of this report a technique was developed in which the coupled-mode two-point boundary value problem is solved exactly by constant-coefficient coupled Riccati equations. The technique is applicable to cases in which arbitrary numbers of space harmonics are included. The constant-coefficient nature of the coupled Riccati equations allows numerical solution of cases in which the exact Riccati equation becomes numerically unstable and therefore we are able to investigate cases heretofore inaccessible. As the coupled Riccati equations are derived from the same set of equations that determine the Brillouin diagram, the predictive ability of the Brillouin diagram is thus illustrated. In the almost periodic case, numerical results illustrate that bandgap regions give rise to frequency regimes of large reflection, as one would expect. The result allows us to use the Brillouin diagram and reflection coefficient interchangeabiy in qualitative discussions of periodic and almost periodic media.

B) How Periodic and Almost Periodic Structures Differ 
1) Phase Relations

As has already been explicitly shown, ${ }^{2}$ different phasings of harmonic perturbations in a periodic structure lead to diverse electromagnetic responses, as evidenced by Brillouin-diagram results. In this report it has been shown explicitly that such is not the case in an almost periodic structure to the most important order of approximation. As precise phasing of multiple harmonic perturbations is a very complex task in practice, we feel that this phasing property of almost periodic structures makes these structures in some sense less abstract models than periodic structures.

2) The Band Coalescence Regime

In this report the cases of periodic and almost periodic band coalescence have been studied in detail. It has been found that very closely spaced periodic perturbations give rise to an exceedingly complex bandstructure which should lead to broadband reflection characteristics. In the almost periodic case the picture is much simpler. As the band centers are brought together the reflection "humps" add in a root-mean-square manner until total coalescence is achieved. Each perturbation (or more correctly each associated backward mode) seems somewhat oblivious to the proximity of the other perturbation, in contradistinction to the periodic case where all the perturbations are coupled and many backward modes become important.

C) The Basic Nature of Almost Periodic Structures

1) Multi-tone Almost Periodic Perturbations 
Generalizing the mapping of Chapter II to the case of $\mathrm{N}$ almost periodic tones, we find the first order dispersion matrix to be

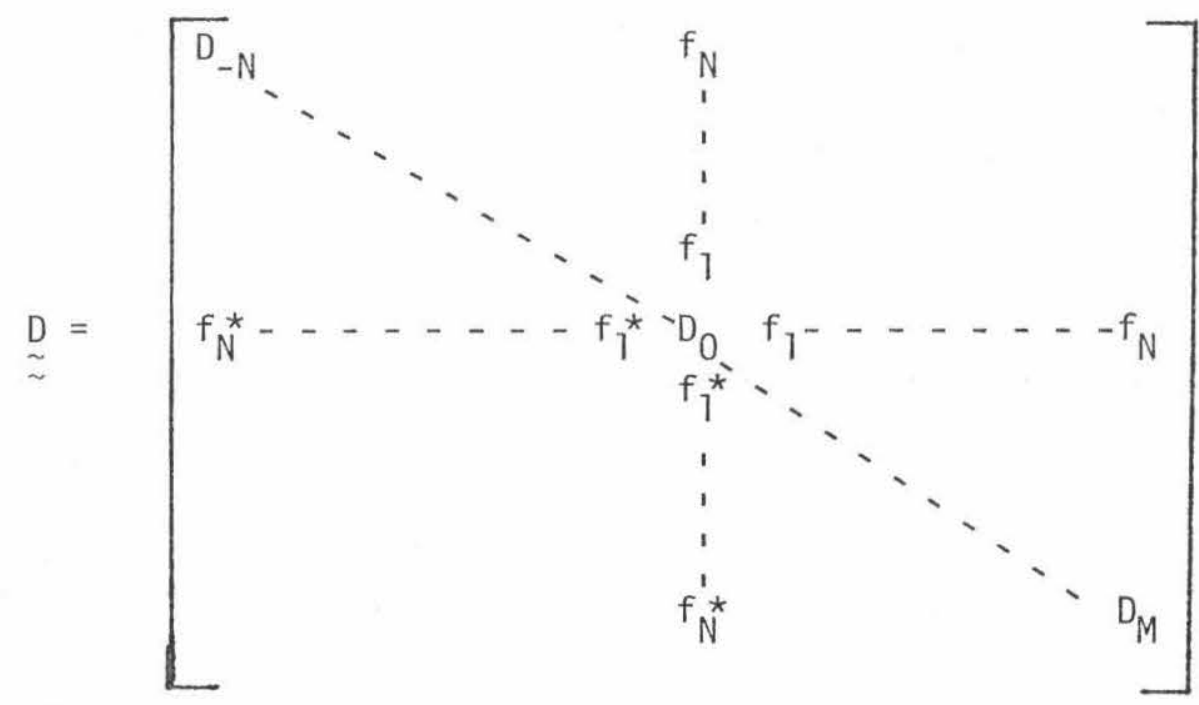

By induction it can be easily shown that the dispersion relation resulting from the determinant of this matrix is again phaseindependent, indicating that the phase independence relation is very basic to almost periodic wave propagation.

2) Almost Periodic Ensembles and Stochastic Ensembles

The apparent incoherency of almost periodic perturbations when coupled together with their root-mean-square addition properties tend to make one think of stochastic effects where phases are averaged and probabilities added. To help force the analogy, we have plotted, in figure (1), the specially constructed AM-FM modulated almost periodic function

$$
\begin{aligned}
& f(z)=\left[1+\frac{1}{6}\left(\cos \kappa_{1} z+\cos \kappa_{2} z+\cos \kappa_{3} z\right)\right] \\
& \times \cos \left[\left(\kappa_{4}+\cos \kappa_{5} z+\cos \kappa_{6} z+\cos \kappa_{7} z\right) z\right]
\end{aligned}
$$




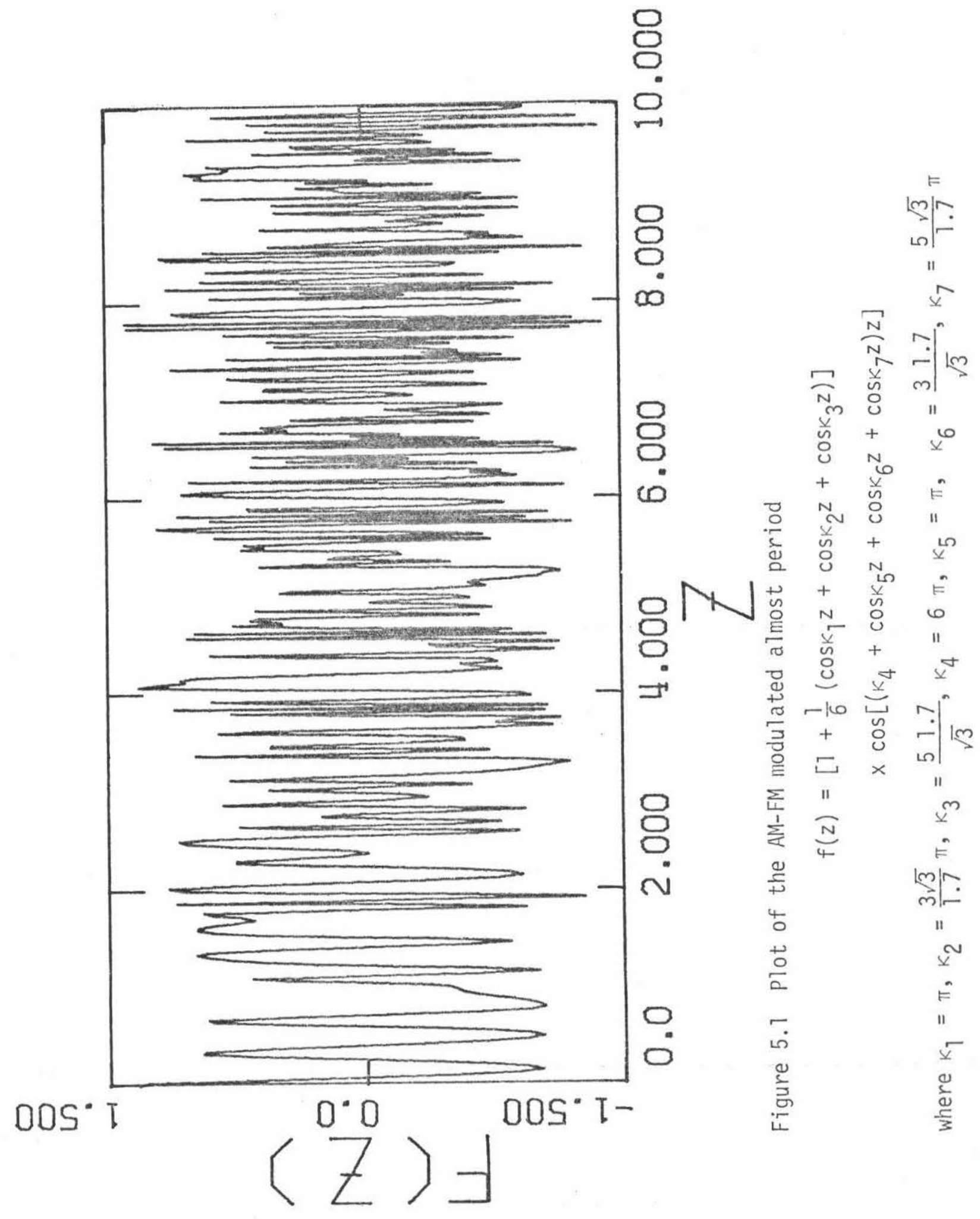


where

$$
\begin{aligned}
\kappa_{1}=\pi & \kappa_{4}=6 . \pi \\
\kappa_{2}=\frac{3 \sqrt{3}}{1.7} \pi & \kappa_{5}=\pi \\
\kappa_{3}=\frac{51.7}{\sqrt{3}} \pi & \kappa_{6}=\frac{31.7}{\sqrt{3}} \pi \\
\kappa_{7}=\frac{5 . \sqrt{3}}{1.7} \pi &
\end{aligned}
$$

(That (2) is almost periodic is evident from elementary AM and FM considerations, i.e., its spectrum is a line spectrum.) Although (2) possesses a line spectrum we could consider the various $k$ 's as random variables which vary between members of an ensemble of functions of the form of (2), and thereby obtain a stationary ensemble of arbitrary spectra. This could certainly be done with other deterministic functions, but we know of none with phase properties of the almost periodic functions. Further, as (2) illustrates we can generate very general almost periodic functions and still have the ability to analyze the wave propagation characteristics in such media through the matrix of (1).

D) Possible Research Directions

1) Extensions to More General Geometries

Certainly the longitudinal geometry is not the only or even the most common wave propagation geometry. By choosing separable geometries and using the solution of Chapter II, a plethora of interesting problems could be solved. A possibility would be the 
transverse geometry of holography analyzed by Burckhardt ${ }^{3}$, The transverse geometry is nice as gratings are both fabricated and operated in such a geometry, a fact which may make that geometry the simplest in which to attempt experimental verification of the almost periodic wave propagation theory. As was mentioned in Chapter I, the three-dimensional geometry in which phonons propagate could also be of interest.

2) Modeling of Random Perturbations

As was discussed in (C) above, almost periodic structures seem to be natural candidates for deterministic members of stochastic ensembles. By using AM, FM and/or more general sums of almost periodic tones, modeling of very general statistical perturbations should be possible.

3) Extensions to Active Media-Stability Criteria

Following the lead of Jaggard and Elachi ${ }^{4}$ and Jaggard ${ }^{5}$, who analyzed the periodic case, one could attempt to analyze the possibly more realistic almost periodic case. Such results could be applicable to distributed feedback lasers.

4) Experimental Verification of the Theory

This would certainly be the most important contribution to the theory. Although the two-tone case discussed in this report would be very hard to verify directly, it seems within the realm of imagination that a more easily verifiable case could be constructed, perhaps from the list of suggested topics above. However, the 
158.

verification could be performed, though care will have to be taken to make sure that it is indeed the difference between periodic and almost periodic media that is the measured quantity. 
Bibligraphy for Chapter Five

1. Various references could be cited on this point. We choose to include those by A. Yariv and the reference therein cited: Yariv, A., Introduction to Optical Electronics (New York: Holt, Rinehart and Winston, 1976). See page 375. See, also: Yariv, A., Quantum Electronics (New York: J. Wiley and Sons, 1975). See page 524.

2. Jaggard, D. L. and C. Elachi, JOSA 66, 674-681, 1976.

3. Burckhardt, C. B., JOSA 56, 1502-1509, 1966.

4. Jaggard, D. L. and C. Elachi, J.A.P. 48, 1461-1466, 1977.

5. Jaggard, D. L., App. Phys. (Springer-Verlag) 13, 185-195, 1977. 
160.

\section{APPENDIX A}

Ordering the Roots of a Third Order Polynomial

We start from the set of equations for the roots of the third order polynomial

$$
z^{3}+a_{2} z^{2}+a_{1} z+a_{0}=0
$$

which can be written as ${ }^{1}$

$$
\begin{aligned}
& z_{1}=s_{1}+s_{2}-\frac{a_{2}}{3} \\
& z_{2}=-\frac{1}{2}\left(s_{1}+s_{2}\right)-\frac{a_{2}}{3}+i \frac{\sqrt{3}}{2}\left(s_{1}-s_{2}\right) \\
& z_{3}=-\frac{1}{2}\left(s_{1}+s_{2}\right)-\frac{a_{2}}{3}-\frac{i \sqrt{3}}{2}\left(s_{1}-s_{2}\right)
\end{aligned}
$$

where

$$
\begin{aligned}
& s_{1}=[r+f]^{1 / 3} \\
& s_{2}=[r-f]^{1 / 3} \\
& f=\left[q^{3}+r^{2}\right]^{1 / 2}
\end{aligned}
$$

and

$$
\begin{aligned}
& q=\frac{1}{3} a_{1}-\frac{1}{9} a_{2}^{2} \\
& r=\frac{1}{6}\left(a_{1} a_{2}-3 a_{0}\right)-\frac{a_{2}^{2}}{27}
\end{aligned}
$$

Using our dispersion relation of Chapter III

$$
\begin{aligned}
& \Delta \beta^{3}+\Delta k \Delta \beta^{2}-\left(\Delta k^{2}+\delta^{2}-\frac{\eta_{+}^{2}}{64}\right) \Delta \beta \\
& -\Delta k\left[\Delta k^{2}-\delta^{2}-\frac{n_{+}^{2}}{64}\right]-\frac{\delta n_{-}^{2}}{64}=0
\end{aligned}
$$


We can make the following identifications:

$$
\begin{aligned}
& q=-\frac{1}{3}\left(\delta^{2}-\frac{n_{+}^{2}}{64}\right)-\frac{4}{9} \Delta k^{2} \\
& r=\Delta k\left[\frac{8}{27} \Delta k^{2}-\frac{2 \delta^{2}}{3}-\frac{1}{3} \frac{n_{+}^{2}}{64}\right]+\frac{\delta n_{-}^{2}}{128}
\end{aligned}
$$

The behavior of these functions is sketched in figure AT.

If we define the quantities

$$
\begin{aligned}
& \delta_{C}=\delta_{\text {critical }}=n_{+}^{2} / 64 \\
& P=8 \delta^{4}+20 \delta^{2} \delta_{C}^{2}-\delta_{C}^{4}
\end{aligned}
$$

we find

$$
F^{2}=-2^{4} \delta^{2}\left[\Delta k^{4}-\frac{\Delta k^{2}}{2^{4} \delta^{2}} P_{\delta}+\frac{\left(\delta^{2}-\delta_{c}^{2}\right)^{3}}{2^{4} \delta^{2}}\right]
$$

We note that the roots of this expression are at

$$
\Delta k^{2}=\frac{1}{2^{5} \delta^{2}}\left[p_{\delta} \pm \sqrt{p_{\delta}^{2}-4\left(\delta^{2}-\delta_{c}^{2}\right)^{6}}\right]
$$

The behavior of $\mathrm{F}^{2}$ is sketched in figure $\mathrm{A} 2$.

The problem with direct evaluation of (3) is the arbitrariness of the phase in taking the cube root. Taking a cube root can put us on any of three branches in the complex plane. We solve this problem by invoking the dubious principle of monotonic phase, i.e., forcing the absolute phase of the expression to increase monotonically. Before directly addressing the branch problem, we summarize the results of our calculations and ad hoc rules using the notation for 
$70 ?$.

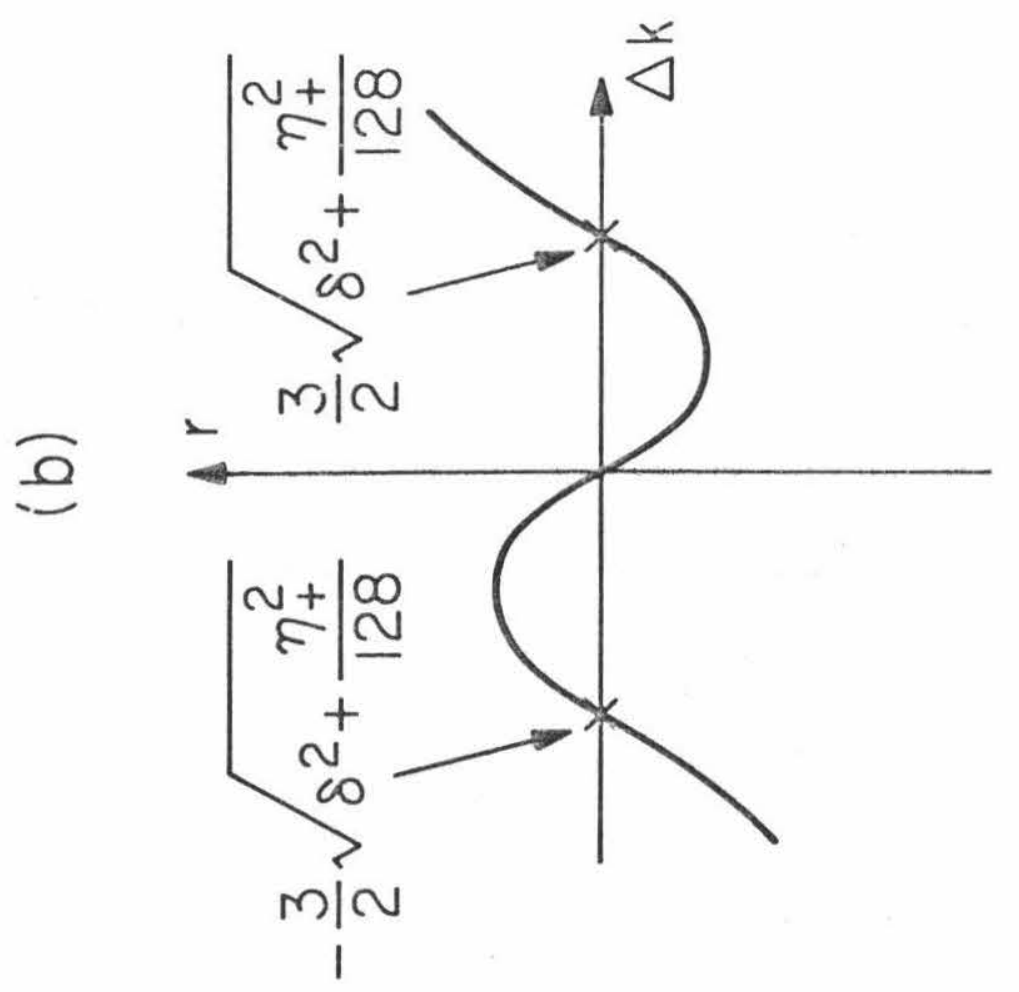

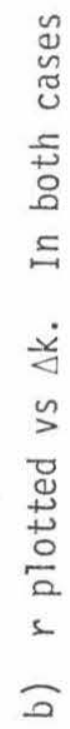
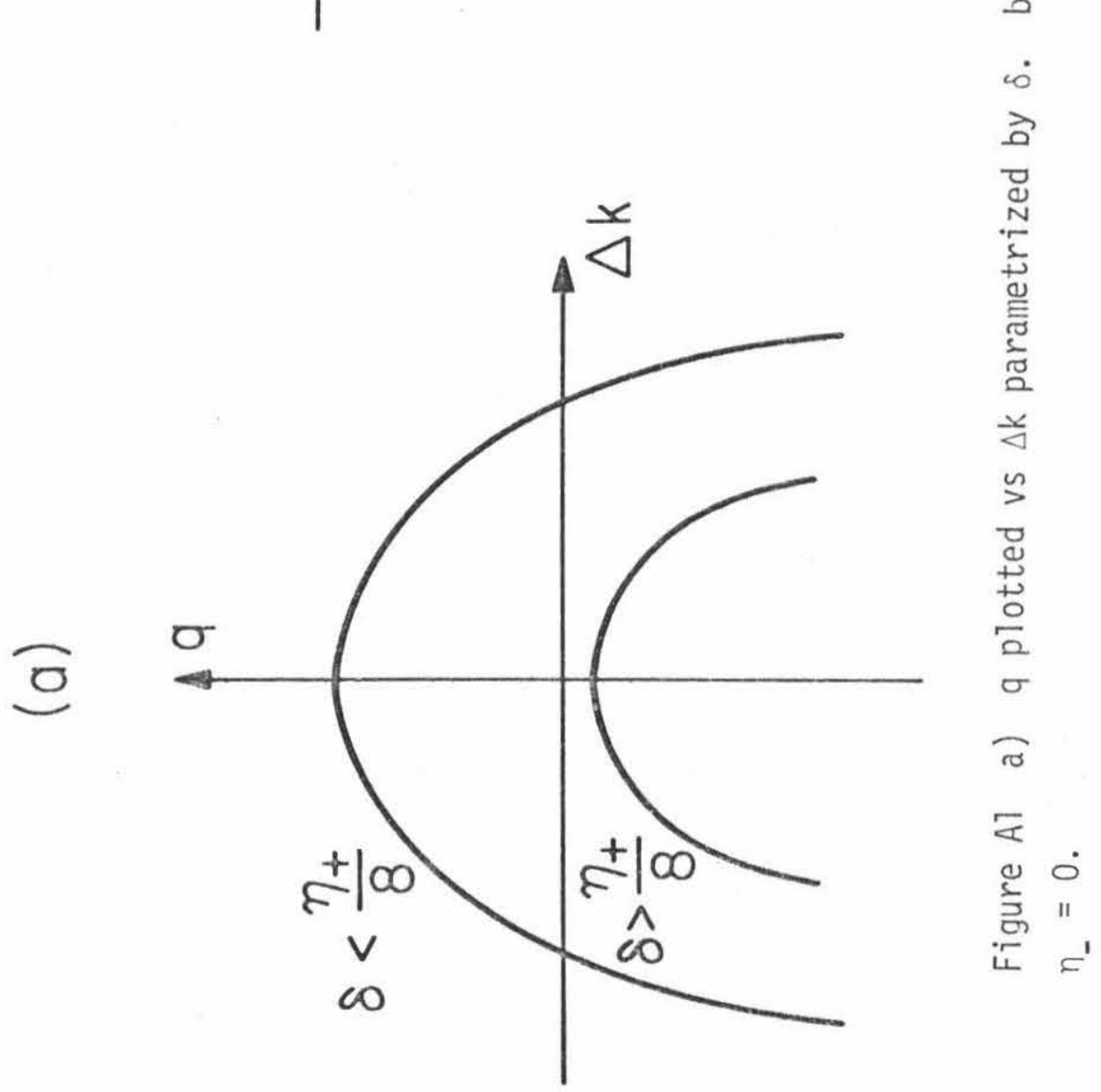
163.

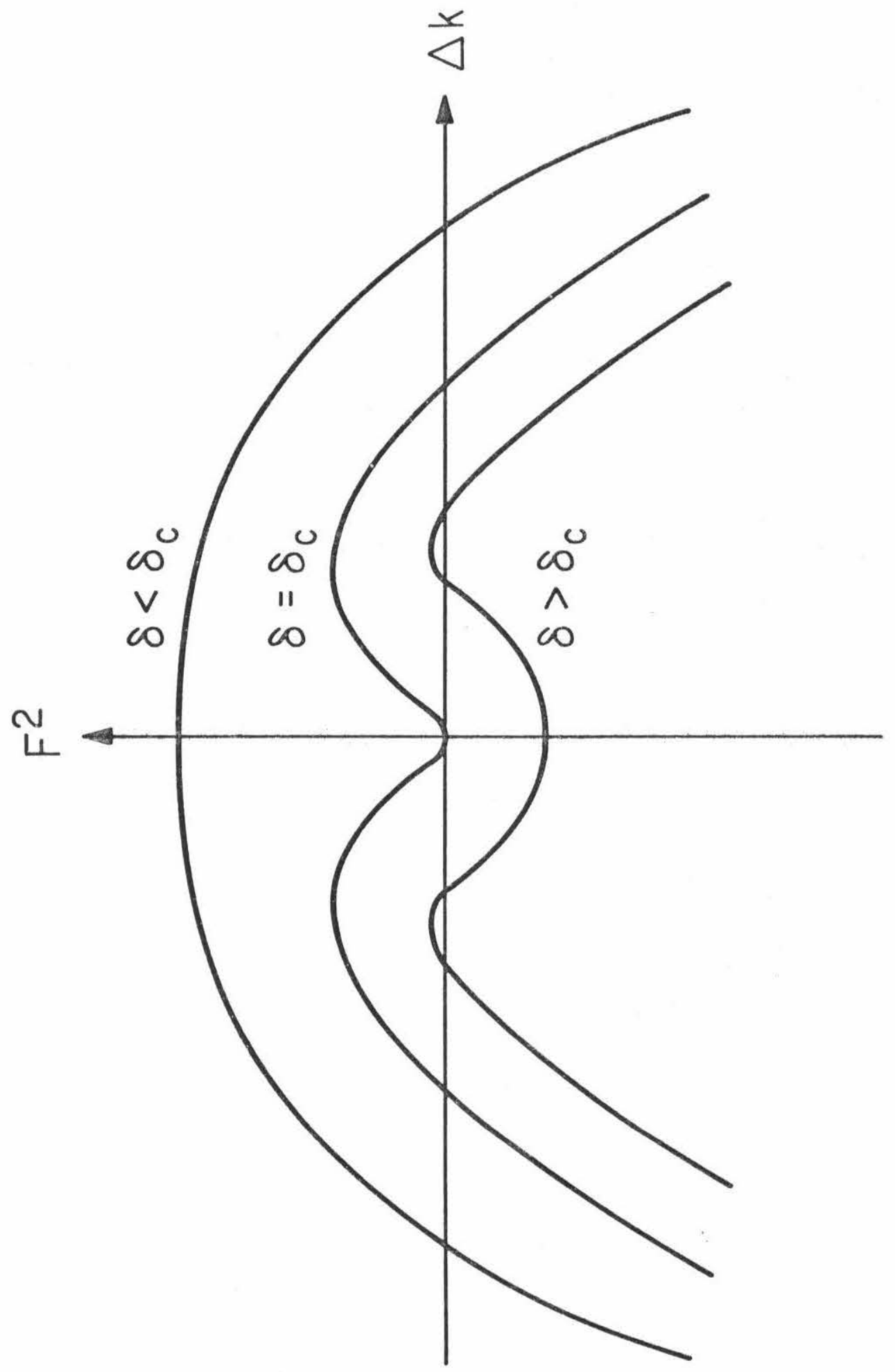

i

$\varepsilon^{\prime}$

茂

莺

.

$\stackrel{\square}{\sqcup}$

is

a

몬

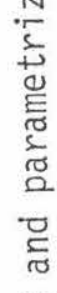

\

$\dot{n}$

离 品

든

$\stackrel{\mathscr{s}}{F}$

₹

흔 
164.

our arbitrary branch variable

$$
\phi=\frac{n \pi}{3}
$$

to find

$$
\begin{aligned}
& \text { Case } 1 \\
& s_{1}=s e^{ \pm i \phi} f^{2}<0 \\
& 2 \\
& z_{1}=2 s \cos \phi \\
& z_{2}=-s \cos \phi-\frac{a_{2}}{3}-\sqrt{3} \sin \phi \\
& z_{3}=-s[\cos \phi-\sqrt{3} \sin \phi]-\frac{a_{2}}{3}
\end{aligned}
$$

\section{Case 2}

$$
\begin{aligned}
& s_{1}=\left|s_{1}\right| e^{i \phi} \quad S P=\left|s_{1}\right|+\left|s_{2}\right| \\
& s_{2}=\left|s_{2}\right| e^{-i \phi} \quad S M=\left|s_{1}\right|-\left|s_{2}\right| \\
& z_{1}=S P \cos \phi-\frac{a_{2}}{3}+i S M \sin \phi \\
& z_{2}=-\frac{S P}{2}(\cos \phi+\sqrt{3} \sin \phi)-\frac{a_{2}}{3}+i \frac{S M}{2}(\sqrt{3} \cos \phi-\sin \phi) \\
& z_{3}=-\frac{S P}{2}(\cos \phi-\sqrt{3} \sin \phi)-\frac{a_{2}}{3}-i \frac{S M}{2}(\sqrt{3} \cos \phi+\sin \phi)
\end{aligned}
$$

Ad hoc rule

If $s_{1}$ and $s_{2}$ have different signs, set

$\left|s_{2}\right|=-\left|s_{2}\right|$

$\rightarrow \mathrm{SP} \rightarrow \mathrm{SM}$

$$
\mathrm{SM} \rightarrow \mathrm{SP}
$$


165.

The branch problem is solved by the following rule (with reference to figure $A 3)$ : Start the phase $3 \phi$ at $3 \pi$ and subtract out $\pi$ for (1) - (2), (4) $\rightarrow$ (5) and (6) $\rightarrow$ (7). 
166.

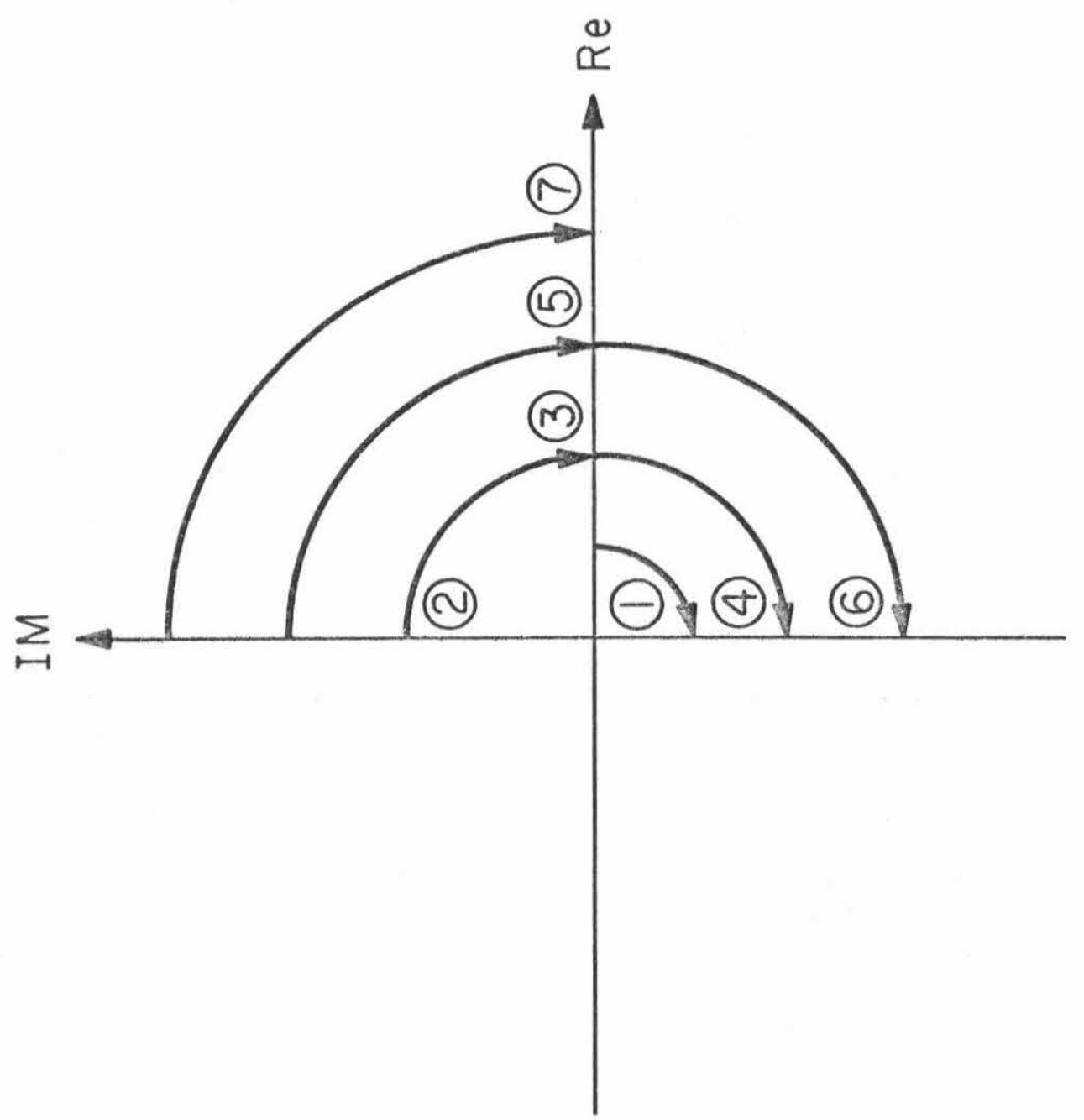

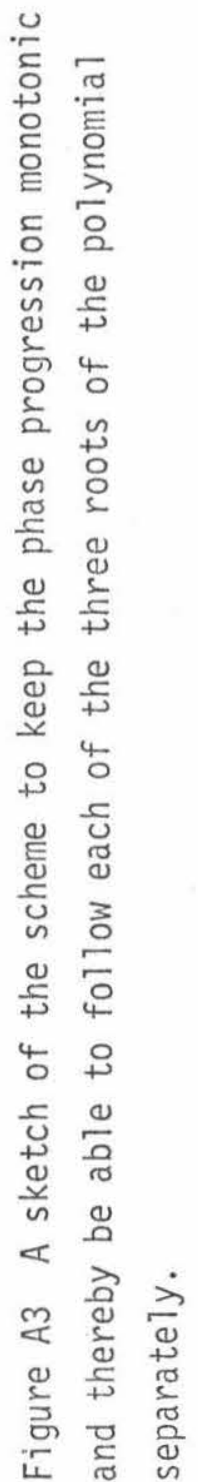


Bibliography for Appendix A

1. Abramovitz, M. and I. Stegan, Handbook of Mathematical Functions, (New York: Dover Pub., Inc., 1970). 
168.

\section{APPENDIX B}

The Constitutive Interaction

We start from the lossless media electrodynamic action (1-2)

$$
s=-\frac{1}{4} \int d^{4} x G^{\alpha \beta_{F}} F_{\alpha \beta}+\int d^{4} x A_{\mu} j_{\nu} n^{\mu \nu}
$$

where

$$
\begin{aligned}
& F_{\mu \nu}=\left[\begin{array}{cccc}
0 & -E_{x} & -E_{y} & -E_{z} \\
E_{x} & 0 & B_{z} & -B_{y} \\
E_{y} & -B_{z} & 0 & B_{x} \\
E_{z} & B_{y} & -B_{x} & 0
\end{array}\right]=A_{\nu, \mu}-A_{\nu, \mu} \\
& G^{\mu \nu}=\left[\begin{array}{cccc}
0 & D_{x} & D_{y} & D_{z} \\
-D_{x} & 0 & H_{z} & -H_{y} \\
-D_{y} & -H_{z} & 0 & H_{x} \\
-D_{z} & H_{y} & -H_{x} & 0
\end{array}\right] \\
& \eta^{\mu \nu}=\left[\begin{array}{cccc}
-1 & 0 & 0 & 0 \\
0 & 1 & 0 & 0 \\
0 & 0 & 1 & 0 \\
0 & 0 & 0 & 1
\end{array}\right] \\
& J^{\mu}=\left[\begin{array}{l}
\rho \\
J
\end{array}\right]
\end{aligned}
$$

and commas denote partial differentiation. It is easily seen that variations performed on (1) yield 


$$
G^{\alpha \beta},{ }_{\beta}=j^{\alpha}
$$

and that the other Maxwell equations

$$
F_{[\alpha \beta, \gamma]}=0
$$

follow from (2a):

We continue by defining the most general lossless, linear constitutive relation by

$$
G^{\mu \nu}=C^{\mu \nu \alpha \beta_{F}} F_{\alpha \beta}=F^{\mu \nu}-M^{\mu \nu}
$$

where

$$
c^{\mu \nu \alpha \beta}=n^{\mu \alpha} n^{\nu \beta}-\frac{1}{2} x^{\mu \nu \alpha \beta}
$$

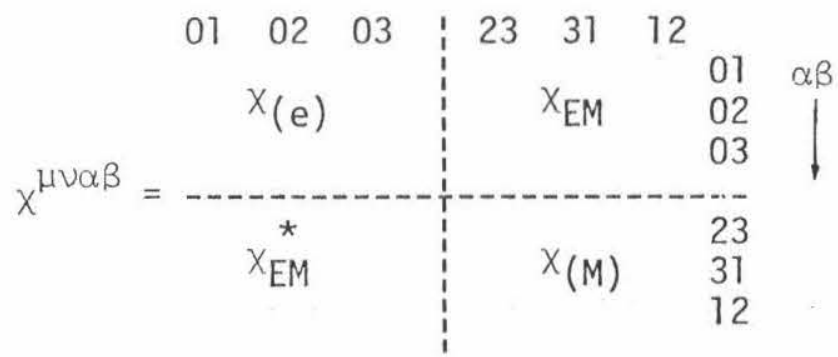

$$
\begin{aligned}
& x_{(e)}=\underset{\sim}{\varepsilon}-\underset{\sim}{\sim}-\underset{\sim}{\sim} \\
& x_{(M)}=\underset{\sim}{I}-\underset{\sim}{\sim}-1 \\
& x^{(\mu \nu) \alpha \beta}=x^{\mu \nu(\alpha \beta)}=x^{(\mu \nu)(\alpha \beta)}=0 \\
& x^{\mu \nu \alpha \beta}=\left(x^{\mu \nu \alpha \beta}\right)^{\star}
\end{aligned}
$$

$$
M^{\mu \nu}=\frac{1}{2} x^{\mu \nu \alpha \beta_{F}} F_{\alpha \beta}=\left[\begin{array}{cccc}
0 & -P_{x} & -P_{y} & -P_{z} \\
P_{x} & 0 & M_{z} & -M_{y} \\
P_{y} & -M_{z} & 0 & M_{x} \\
P_{z} & M_{y} & -M_{x} & 0
\end{array}\right]
$$

With the above substitutions, we can rewrite our action in the form 


$$
\begin{aligned}
S= & -\frac{1}{4} \int d^{4} \times \eta^{\mu \alpha} \eta^{\nu \beta_{F}} F_{\alpha \beta} F_{\mu \nu}+\frac{1}{8} \int d^{4} \times x^{\mu \nu \alpha \beta} F_{\mu \nu} F_{\alpha \beta} \\
& +\int d^{4} \times A_{\mu} j_{\nu} n^{\mu \nu}
\end{aligned}
$$

If we perform variations with respect to $A_{\mu}$ on (5), we find the corresponding Maxwell equations

$$
F^{\mu \nu}, \nu=M^{\mu \nu}, \nu+j^{\mu}=j_{\text {bound }}^{\mu}+j_{\text {free }}^{\mu}
$$

with the corresponding interpretation that the $\mathrm{E}$ and $\mathrm{B}$ travel through free space augmented by a bound polarization current. This can be explicitly illustrated by integrating the second term of (5) by parts, to obtain

$$
\begin{aligned}
& \frac{1}{8} \int d^{4} \times x^{\mu \nu \alpha \beta_{F}} F_{\mu \nu} F_{\alpha \beta}=\int d^{4} \times M^{\mu \nu}, \nu_{\mu}^{A}=\int d^{4} \times A_{\alpha} j_{\nu}^{\text {bound }} \eta^{\alpha \nu} \\
& \text { Our argument can now be completed by noting that }
\end{aligned}
$$

Scattered fields a transition amplitude $T \alpha$ interaction action $S_{I} \cdot(8)$

Certainly for the first-order interaction with a lossless, linear constitutive media,

$$
S_{I}=\int d^{4} \times A_{\alpha} j_{v}^{\text {bound }} n^{\alpha \nu} \text {. }
$$

Taking the incoming and outgoing states to be plane wave states, incoming of propagation vector ${ }^{0} \mathrm{~K}$, polarization ${ }^{0} \mathrm{E}$, outgoing of propagation vector ${ }^{1} k$, polarization ${ }^{1} \varepsilon$, we evaluate (9) to find (after some manipulation) 


$$
\left.T \alpha{ }^{1} k_{\alpha}{ }^{1} \varepsilon_{\mu}{ }^{f} k\left(x^{\alpha \mu \beta \nu}\right)\right|_{k={ }^{1} k-{ }^{0} k}{ }^{0} k_{B}{ }^{0} \varepsilon_{v} .
$$

The result is manifestly gauge invariant under transformations

$$
\begin{aligned}
& { }^{1} \varepsilon_{\mu} \rightarrow{ }^{1} \varepsilon_{\mu}+\gamma{ }^{1} k_{\mu} \\
& { }^{0} \varepsilon_{\mu} \rightarrow{ }^{0} \varepsilon_{\mu}+\gamma{ }^{0} k_{\mu}
\end{aligned}
$$

and explicitly illustrates that any first-order interaction with a lossless, linear constitutive media is proportional to the Fourier transform of the medium's constitutive tensor. The argument can be carried to higher orders and can be shown to be equivalent to the Neumann series for simple media. 


\section{APPENDIX C \\ The Electromagnetic Riccati Equation}

We first address the question of what the basic properties of the Riccati equation are, or more specifically whether the individual terms have meaning. Starting from the equation itself

$$
\frac{d R}{d z}=\frac{1}{2}\left(1-R^{2}\right) \frac{d k}{d z} \frac{1}{k}-2 i k R
$$

We first consider the case where

$$
\frac{1}{\mathrm{k}} \frac{\mathrm{dk}}{\mathrm{dz}}<k \text {. }
$$

Equation (1) then becomes

$$
\frac{d R}{d z}=-2 i k R
$$

with the obvious solution

$$
R=R_{0} e^{-2 i \int_{0}^{z} k\left(z^{\prime}\right) d z^{\prime}}
$$

The clear interpretations of (4) are that the last term on the right hand side of (1) must be some sort of phase determining quantity, and that the magnitude of the reflection coefficient is dependent not so much on $k$ as on its rate of change. We now investigate the first term of (1) by making the assumption that

$$
k<\frac{1}{k} \frac{d k}{d z}
$$

In this case the second term on the right hand side of (1) is ignorable and (1) can be integrated to yield

$$
R=\frac{k_{f}-k_{0}+R_{0}\left(k_{f}+k_{0}\right)}{k_{f}+k_{0}+R_{0}\left(k_{f}-k_{0}\right)}
$$


This result contains as limits the two well-known results that as

$$
\begin{aligned}
R_{0} & \rightarrow 0 \\
R & \rightarrow \frac{n-n_{0}}{n+n_{0}}
\end{aligned}
$$

and as

$$
\begin{aligned}
& R_{0} \rightarrow 1 \\
& R \rightarrow 1
\end{aligned}
$$

and therefore shows that the first term on the right hand side of (1) contains the boundary effects. This is reasonable as the condition (5) is satisfied only in the neighborhood of sharp interfaces.

There exist many perturbation schemes other than the BornNeumann series discussed in Chapter IV. Examples are the Bremmer series $^{1}$ and the Arth $(R)$ transformation ${ }^{2}$. By considering explicitly the behavior of these approximation schemes in periodic media it is easily seen that they become secular. Here we wish to present a simple argument to show that any perturbation scheme which uses simple ordering of terms will become secular at resonance. Let us write the Riccati equation in the form

$$
\frac{d R}{d z}=\frac{1}{2}\left(1-R^{2}\right) \frac{d}{d z}(\log n(z))-2 i k n(z) R
$$

where

$$
k=k_{0}=\omega / c
$$

and we take the index of refraction to be of the form 


$$
n=n_{0}[1+n P(z)]
$$

where $\mathrm{n}$ is small and $\mathrm{P}$ denotes a periodic function. We now Fourier transform (9) into the variable $\beta$ to obtain

$$
-i \beta R=-\frac{i \beta}{2} F_{\beta}[\log n]+\frac{i}{2} \hat{R} * \hat{R} * \beta f_{\beta}[\log n]-2 i k f_{\beta}[n] * \hat{R}
$$

where the asterisk denotes convolution. We now expand

$$
\begin{aligned}
\log n & =\log n_{0}+\log [1+n P] \\
& =\log n_{0}+\sum_{n=0}^{\infty}(-)^{n} \frac{(n P)^{n+1}}{n+1} \\
& =\log n_{0}+\sum=\alpha+\sum
\end{aligned}
$$

With this we find

$$
\begin{aligned}
& f_{\beta}[\log n]=\alpha \delta(\beta)+\hat{\Sigma} \\
& f_{\beta}[n]=n_{0} \delta(\beta)+n_{0} n \hat{P}
\end{aligned}
$$

Substituting (14) in (12) we find

$$
\left[2 k n_{0}-\beta\right] R=-\frac{\beta}{2} \hat{\Sigma}+\frac{1}{2} \hat{R} *\left[\hat{R} * \beta \hat{\Sigma}-2 k_{0} n_{0} n \hat{P}\right]
$$

The Bragg condition is just

$$
2 k n_{0}=\beta
$$

showing that the left-hand side of (15) vanishes on resonance. But the left-hand side is the only zeroth-order term in $n$ of (15). Clearly, for this reason, any expansion in $n$ of (9) must fail as the higher order terms in $n$ become more important than the zeroth-order term at resonance. 
Bibliography for Appendix C

1. The most recent reference is: Bellman, R. and G. M. Wing, An Introduction to Invariant Imbedding (New York: J. Wiley and Sons, 1975). The original reference is: K1 ine, M. (Ed.), The Theory of Electromagnetic Waves (New York: Dover Pub., Inc., 1951). See pages 169180.

2. There are two applicable papers in the Russian literature:

Gaydabura, I. S., Radio Eng. Electron. Phys. 16, No. 10, 1625-1627, 1971 and Gaydabura, I. S. and I. A. Kozlov, Radio Eng. Electron. Phys. 18, 1747-1749, 1973. 
APPENDIX D

Two-Point Boundary Value Problems

We consider the geometry of figure (4.1), Chapter IV. We wish to find the transmission and reflection coefficients of the system defined by

$$
\begin{aligned}
& \frac{\mathrm{dF}(z)}{\mathrm{dz}}-\underset{\sim}{i}{\underset{\sim}{F}}_{\sim} F(z)=\underset{\sim}{i \underset{\sim}{\mathrm{X}} \mathrm{FB}} \underset{\sim}{\mathrm{B}}(\mathrm{z}) \\
& \frac{\mathrm{d} B(z)}{\tilde{d} z}-\underset{\sim}{i} \delta_{\sim} \underset{\sim}{B}(z)=\underset{\sim}{i \underset{\sim}{x}} \underset{\sim}{F}(z)
\end{aligned}
$$

where $\underset{\sim}{F}$ is an $N$ vector and $\underset{\sim}{B}$ an $M$ vector. As the system of (1) is always reducible to an $(N+M)^{\text {th }}$ order differential equation, we can assume solutions of the form

$$
\begin{aligned}
& \underset{\sim}{F}(z)=\sum_{i=1}^{N+M} \underset{\sim j}{f} e^{i D_{i} z} \\
& \underset{\sim}{B}(z)=\sum_{i=1}^{N+M}{\underset{\sim}{b}}_{j} e^{i D_{j} z}
\end{aligned}
$$

where the $D i$ 's must be determined as the roots of an $(M+N)$ th order polynomial. We can substitute (2) back into (1) and obtain $2 \mathrm{~N}+2 \mathrm{M}$ equations for the $f i$ 's and the bi's. However, only $(\mathrm{N}+\mathrm{M})$ of these equations will be independent. To complete the solution to our prob1 em we must write the system

$$
\begin{array}{ll}
\underset{\sim}{\mathrm{F}}\left(-\frac{\ell}{2}\right)={\underset{\sim}{\mathrm{i}}}_{1} & \underset{\sim}{\mathrm{F}}\left(\frac{\ell}{2}\right)=\underset{\sim}{\mathrm{T}} \\
\underset{\sim}{\mathrm{B}}\left(-\frac{\ell}{2}\right)=\underset{\sim}{\mathrm{R}} & \underset{\sim}{\mathrm{B}}\left(\frac{\ell}{2}\right)=\underset{\sim}{0}
\end{array}
$$


where $e_{i}$ is a unit input vector. The system of (3) represents $2 N+2 M$ equations for $3 N+3 M$ unknowns: $\underset{\sim}{F}, \underset{\sim}{B}, \underset{\sim}{T}, \underset{\sim}{R}$. But when coupled with the $\mathrm{N}+\mathrm{M}$ independent equations obtained from substituting (2) in (1), we are left with a $3(N+M)$ order system in $3(\mathrm{~N}+\mathrm{M})$ unknowns, and therefore can compute a unique solution for $\underset{\sim}{R}$ and $\underset{\sim}{T}$. To summarize, solution of the two-point boundary value problem requires: 1$)$ derivation of the $(N+M)$ th-order differential equation from the system of $(1) ; 2$ ) determination of the roots of the $(\mathrm{N}+\mathrm{M})$ th-order polynomial resulting from the $(\mathrm{N}+\mathrm{M})$ th-order differential equation; 3 ) solution of a $3(\mathrm{~N}+\mathrm{M})$ order system of algebraic equations. 


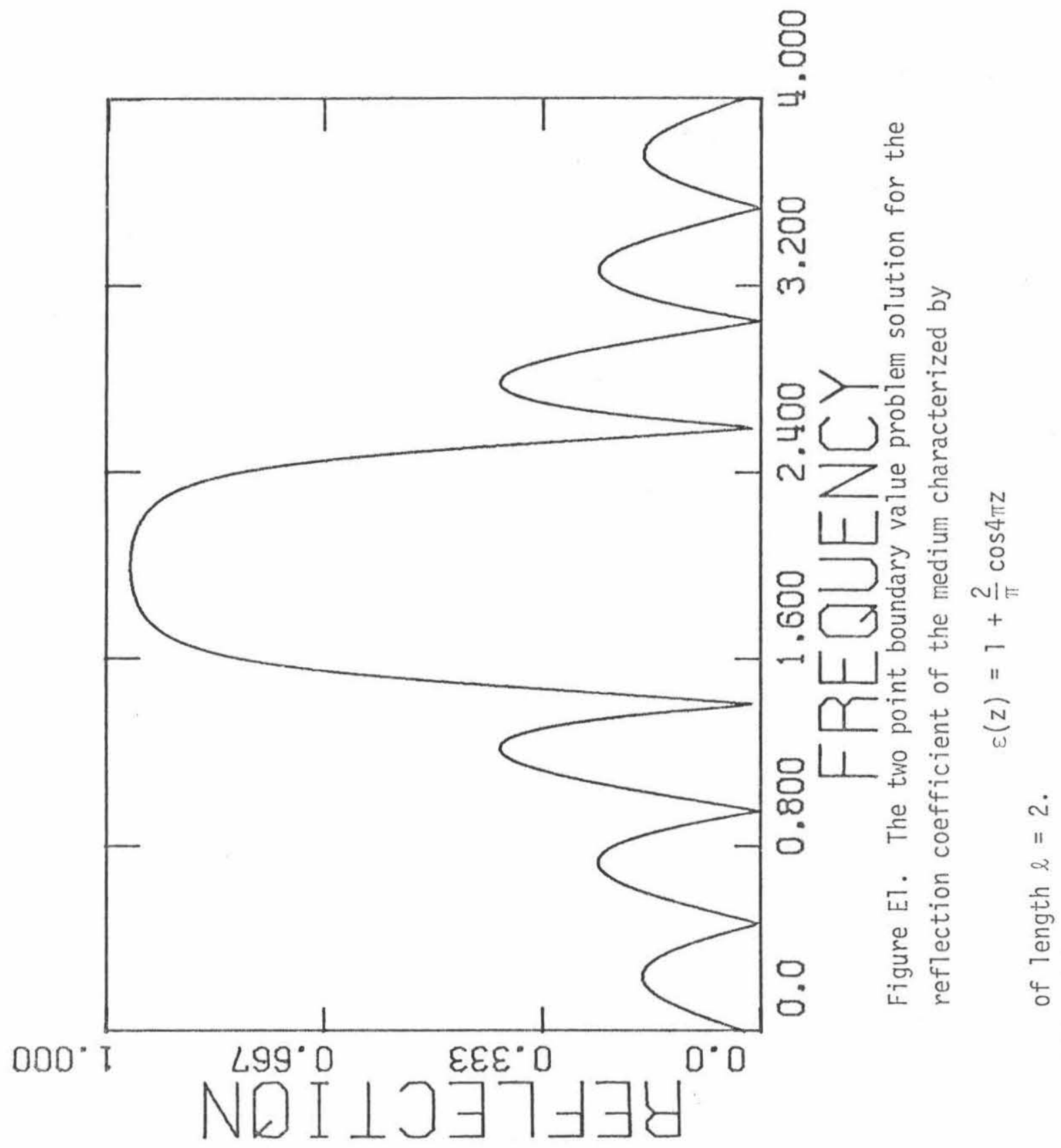


130.

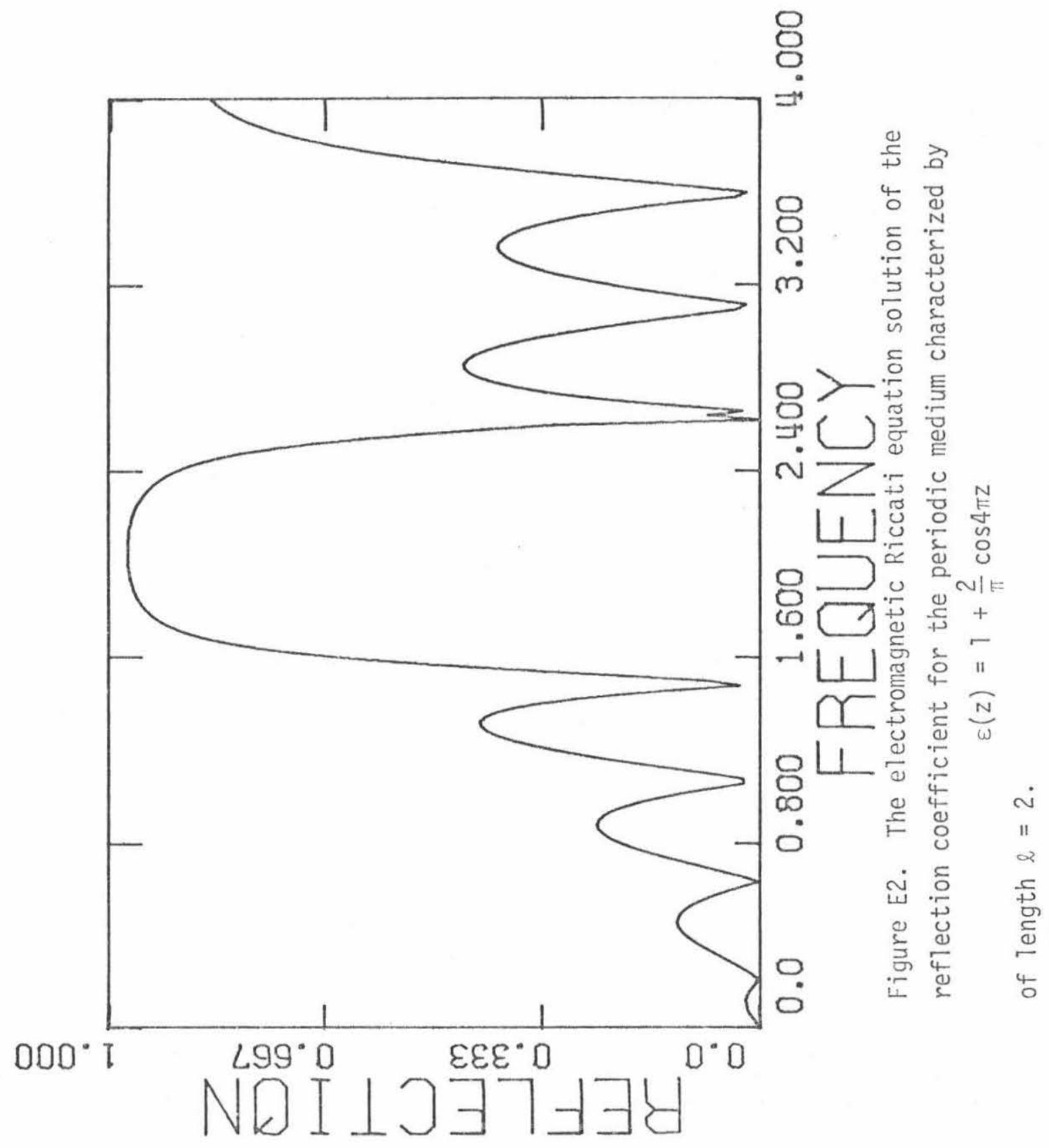

